\title{
Topical antihistamines and mast cell stabilisers for treating seasonal and perennial allergic conjunctivitis (Review)
}

\author{
Castillo M, Scott NW, Mustafa MZ, Mustafa MS, Azuara-Blanco A
}

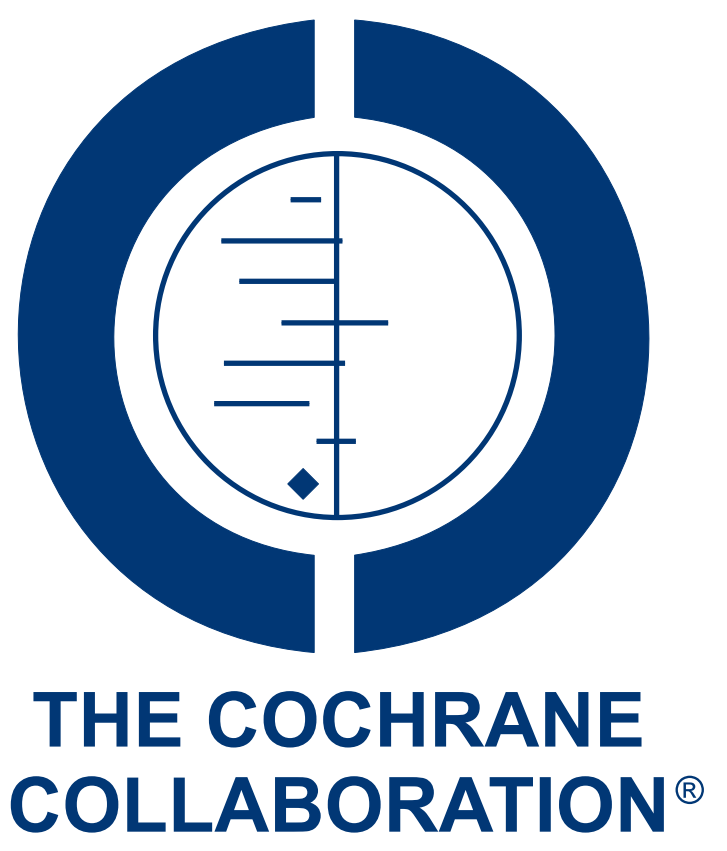

This is a reprint of a Cochrane review, prepared and maintained by The Cochrane Collaboration and published in The Cochrane Library 2015, Issue 6

http://www.thecochranelibrary.com

\section{WILEY}

Topical antihistamines and mast cell stabilisers for treating seasonal and perennial allergic conjunctivitis (Review)

Copyright $\odot 2015$ The Cochrane Collaboration. Published by John Wiley \& Sons, Ltd. 
TABLE OF CONTENTS

HEADER . . . . . . . . . . . . . . . . . . . . . . . . . . . . . . . . . . . . . . . 1

ABSTRACT . . . . . . . . . . . . . . . . . . . . . . . . . . . . . . . . . . . . . . . . 1

PLAIN LANGUAGE SUMMARY . . . . . . . . . . . . . . . . . . . . . . . . . . . . . . . . . . . . . . .

BACKGROUND . . . . . . . . . . . . . . . . . . . . . . . . . . . . . . . . . . . . . . .

OBJECTIVES . . . . . . . . . . . . . . . . . . . . . . . . . . . . . . . . . . . . . . . . 44

METHODS . . . . . . . . . . . . . . . . . . . . . . . . . . . . . . . . . . . . . . . 4.4

RESULTS . . . . . . . . . . . . . . . . . . . . . . . . . . . . . . . . . . . . . . . 6

Figure 1. . . . . . . . . . . . . . . . . . . . . . . . . . . . . . . . . . 8

Figure 2. . . . . . . . . . . . . . . . . . . . . . . . . . . . . . . . . . . . . . 9

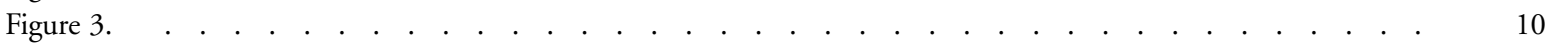

Figure 4. . . . . . . . . . . . . . . . . . . . . . . . . . . . . . . . . . . . . 11

Figure 5. . . . . . . . . . . . . . . . . . . . . . . . . . . . . . . . . . . . . . 20

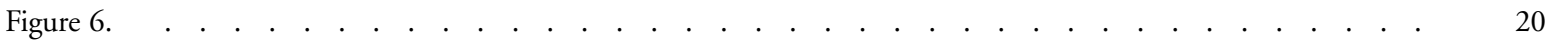

DISCUSSION . . . . . . . . . . . . . . . . . . . . . . . . . . . . . . . . . . . . . 29

AUTHORS' CONCLUSIONS . . . . . . . . . . . . . . . . . . . . . . . . . . . . . . . . 29

ACKNOWLEDGEMENTS . . . . . . . . . . . . . . . . . . . . . . . . . . . . . . . . . 29

REFERENCES . . . . . . . . . . . . . . . . . . . . . . . . . . . . . . . . . . . . . . 30

CHARACTERISTICS OF STUDIES . . . . . . . . . . . . . . . . . . . . . . . . . . . . . . . . 33

DATA AND ANALYSES . . . . . . . . . . . . . . . . . . . . . . . . . . . . . . . . . . . . . . . . . . . . . . . . . . .

Analysis 1.1. Comparison 1 Olopatadine versus ketotifen, Outcome 1 Itching at 14 days. . . . . . . . . . . . . 69

Analysis 1.2. Comparison 1 Olopatadine versus ketotifen, Outcome 2 Tearing at 14 days. . . . . . . . . . . . . 70

ADDITIONAL TABLES . . . . . . . . . . . . . . . . . . . . . . . . . . . . . . . . . . 70

APPENDICES . . . . . . . . . . . . . . . . . . . . . . . . . . . . . . . . . . . . . 72

CONTRIBUTIONS OF AUTHORS . . . . . . . . . . . . . . . . . . . . . . . . . . . . . . . . . . . . . . . . . . . .

DECLARATIONS OF INTEREST . . . . . . . . . . . . . . . . . . . . . . . . . . . . . . . . . . . 76

SOURCES OF SUPPORT . . . . . . . . . . . . . . . . . . . . . . . . . . . . . . . . . . . . . . . . . . . . . . . .

DIFFERENCES BETWEEN PROTOCOL AND REVIEW . . . . . . . . . . . . . . . . . . . . . . . . . . . . 77

NOTES . . . . . . . . . . . . . . . . . . . . . . . . . . . . . . . . . . . . . . . . . . . . 77

Topical antihistamines and mast cell stabilisers for treating seasonal and perennial allergic conjunctivitis (Review)

Copyright $\odot 2015$ The Cochrane Collaboration. Published by John Wiley \& Sons, Ltd. 


\title{
[Intervention Review]
}

\section{Topical antihistamines and mast cell stabilisers for treating seasonal and perennial allergic conjunctivitis}

\author{
Mayret Castillo ${ }^{1}$, Neil W Scott ${ }^{2}$, Mohammad Z Mustafa ${ }^{3}$, Mohammed S Mustafa ${ }^{4}$, Augusto Azuara-Blanco ${ }^{5}$ \\ ${ }^{1}$ Queen's University Belfast, Belfast, UK. ${ }^{2}$ Medical Statistics Team, University of Aberdeen, Aberdeen, UK. ${ }^{3}$ Princess Alexandra Eye \\ Pavilion, Edinburgh, UK. ${ }^{4}$ Moorfields Eye Hospital Dubai, Dubai, United Arab Emirates. ${ }^{5}$ Centre for Experimental Medicine, Queen's \\ University Belfast, Belfast, UK \\ Contact address: Augusto Azuara-Blanco, Centre for Experimental Medicine, Queen's University Belfast, Grosvenor Road, Belfast, \\ BT12 6BA, UK. aazblanco@aol.com. a.azuara-blanco@qub.ac.uk.
}

Editorial group: Cochrane Eyes and Vision Group.

Publication status and date: New, published in Issue 6, 2015.

Review content assessed as up-to-date: 17 July 2014.

Citation: Castillo M, Scott NW, Mustafa MZ, Mustafa MS, Azuara-Blanco A. Topical antihistamines and mast cell stabilisers for treating seasonal and perennial allergic conjunctivitis. Cochrane Database of Systematic Reviews 2015, Issue 6. Art. No.: CD009566. DOI: 10.1002/14651858.CD009566.pub2.

Copyright (C) 2015 The Cochrane Collaboration. Published by John Wiley \& Sons, Ltd.

\begin{abstract}
A B S T R A C T
Background

Seasonal/perennial allergic conjunctivitis is the most common allergic conjunctivitis, usually with acute manifestations when a person is exposed to allergens and with typical signs and symptoms including itching, redness, and tearing. The clinical signs and symptoms of allergic conjunctivitis are mediated by the release of histamine by mast cells. Histamine antagonists (also called antihistamines) inhibit the action of histamine by blocking histamine $\mathrm{H} 1$ receptors, antagonising the vasoconstrictor, and to a lesser extent, the vasodilator effects of histamine. Mast cell stabilisers inhibit degranulation and consequently the release of histamine by interrupting the normal chain of intracellular signals.
\end{abstract}

Topical treatments include eye drops with antihistamines, mast cell stabilisers, non-steroidal anti-inflammatory drugs, combinations of the previous treatments, and corticosteroids. Standard treatment is based on topical antihistamines alone or topical mast cell stabilisers alone or a combination of treatments. There is clinical uncertainty about the relative efficacy and safety of topical treatment.

Objectives

The objective of this review was to assess the effects of topical antihistamines and mast cell stabilisers, alone or in combination, for use in treating seasonal and perennial allergic conjunctivitis.

\section{Search methods}

We searched CENTRAL (which contains the Cochrane Eyes and Vision Trials Register) (2014, Issue 7), Ovid MEDLINE, Ovid MEDLINE In-Process and Other Non-Indexed Citations, Ovid MEDLINE Daily, Ovid OLDMEDLINE (January 1946 to July 2014), EMBASE (January 1980 to July 2014), the metaRegister of Controlled Trials ( $m$ RCT) (www.controlled-trials.com), ClinicalTrials.gov (www.clinicaltrials.gov) and the World Health Organization (WHO) International Clinical Trials Registry Platform (ICTRP) (www.who.int/ictrp/search/en). We did not use any date or language restrictions in the electronic searches for trials. We last searched the electronic databases on 17 July 2014. We also searched the reference lists of review articles and relevant trial reports for details of further relevant publications.

Topical antihistamines and mast cell stabilisers for treating seasonal and perennial allergic conjunctivitis (Review)

Copyright () 2015 The Cochrane Collaboration. Published by John Wiley \& Sons, Ltd. 


\section{Selection criteria}

We included randomised controlled trials (RCTs) comparing topical antihistamine and mast cell stabilisers, alone or in combination, with placebo, no treatment or to any other antihistamine or mast cell stabiliser, or both, that examined people with seasonal or perennial allergic conjunctivitis, or both. The primary outcome was any participant-reported evaluation (by questionnaire) of severity of four main ocular symptoms: itching, irritation, watering eye (tearing), and photophobia (dislike of light), both separately and, if possible, by an overall symptom score. We considered any follow-up time between one week and one year.

\section{Data collection and analysis}

Two review authors independently extracted data and assessed risk of bias. Disagreements were resolved by discussion among review authors and the involvement of a third review author. We followed standard methodological approaches used by Cochrane.

\section{Main results}

We identified 30 trials with a total of 4344 participants randomised, with 17 different drugs or treatment comparisons. The following antihistamines and mast cell stabilisers were evaluated in at least one RCT: nedocromil sodium or sodium cromoglycate, olopatadine, ketotifen, azelastine, emedastine, levocabastine (or levocabastine), mequitazine, bepotastine besilate, combination of antazoline and tetryzoline, combination of levocabastine and pemirolast potassium. The most common comparison was azelastine versus placebo (nine studies).

We observed a large variability in reporting outcomes. The quality of the studies and reporting was variable, but overall the risk of bias was low. Trials evaluated only short-term effects, with a range of treatment of one to eight weeks. Meta-analysis was only possible in one comparison (olopatadine versus ketotifen). There was some evidence to support that topical antihistamines and mast cell stabilisers reduce symptoms and signs of seasonal allergic conjunctivitis when compared with placebo. There were no reported serious adverse events related to the use of topical antihistamine and mast cell stabilisers treatment.

\section{Authors' conclusions}

It seems that all reported topical antihistamines and mast cell stabilisers reduce symptoms and signs of seasonal allergic conjunctivitis when compared with placebo in the short term. However, there is no long-term data on their efficacy. Direct comparisons of different antihistamines and mast cell stabilisers need to be interpreted with caution. Overall, topical antihistamines and mast cell stabilisers appear to be safe and well tolerated. We observed a large variability in outcomes reported. Poor quality of reporting challenged the synthesis of evidence.

\section{PLAIN LANGUAGE SUMMARY}

Topical antihistamines and mast cell stabilisers for treating seasonal and perennial allergic conjunctivitis

\section{Review question}

Are treatments with eye drops of antihistamines and mast cell stabilisers, alone or in combination, effective and safe in people with seasonal and allergic conjunctivitis? The main outcome measure was eye symptoms reported by participants, including eye itching, irritation (burning sensation), watering eyes (tearing), and photophobia (dislike of light). We found 30 trials.

\section{Background}

Conjunctivitis refers to inflammation of the conjunctiva, which is the thin tissue that covers the sclera (white part of the eye). Seasonal and perennial allergic conjunctivitis is the most common type of allergic conjunctivitis. Although this condition does not cause sight loss, it can cause intense itching and eye watering. Eye drops with antihistamines or mast cell stabilisers, or both are commonly used.

\section{Study characteristics}

The evidence is current to July 2014. Among the 30 studies reviewed there were 17 different comparisons, including 4344 participants ranging in age between 4 and 85 years. The duration of treatment ranged from one to eight weeks. Ten out of 30 studies were funded by the drug manufacturer ( 8 totally and 2 partially funded); 20 studies did not report any source of funding. There was inconsistency in the way the effect of treatment was measured and reported. Overall risk of bias was low.

\section{Key results}

Topical antihistamines and mast cell stabilisers for treating seasonal and perennial allergic conjunctivitis (Review)

Copyright @ 2015 The Cochrane Collaboration. Published by John Wiley \& Sons, Ltd. 
Topical antihistamines and mast cell stabilisers, alone or in combination, are safe and effective for reducing symptoms of seasonal and perennial allergic conjunctivitis. We found insufficient evidence to discern which topical antihistamines and mast cell stabilisers are the most effective.

\section{B A C K G ROU N D}

\section{Description of the condition}

The conjunctiva forms the mucous membrane component of the ocular surface and is continually exposed to a wide variety of allergens. In sensitised individuals, pollen, plant, house dust mite or animal dander allergens dissolve in the tear film, cross the conjunctival epithelium, and activate mast cells in the substantia propria leading to the prevalent and unpleasant conditions known as seasonal allergic conjunctivitis (SAC) and perennial allergic conjunctivitis (PAC) (Anderson 2001).

SAC is the most common allergic conjunctivitis, usually with an acute or subacute manifestation characterised by peaks of self limiting signs and symptoms that become persistent in repeated allergen stimulations during the pollen season. The hallmark signs and symptoms are itching, redness, and lid swelling. Patients may also complain of epiphora (watering eye), mucous (translucent) discharge, rhinitis (runny or stuffy nose), and eye-burning sensation (Leonardi 2008).

In PAC, the non-specific signs and symptoms of redness, burning, and chemosis (swelling of the conjunctiva) may persist with varying severity for months, accompanied by a low level of itching (Leonardi 2008).

Seasonal and perennial allergic conjunctivitis rarely cause visual disability.

\section{Epidemiology}

SAC is the most common allergic eye disorder, affecting up to $20 \%$ of the population in the United Kingdom at some point in their lives (Buckley 1998; Dart 1986). It has a varying prevalence in countries throughout the world (Beasley 1998). PAC is much rarer and typically results in milder symptoms than those seen in the seasonal condition. People with allergic conjunctivitis often have a personal history, family history, or both of atopic disorders. A recent epidemiological survey noted that up to $40 \%$ of 13 and 14-year-olds worldwide reported the symptoms of allergic conjunctivitis (see 'Clinical presentation' below) (Strachan 1997). It has been estimated that ocular symptoms are present in $40 \%$ to $60 \%$ of the allergic population (people with atopy, that is asthma and eczema) (Ono 2005). Visual loss and severe complications are rare.

\section{Biological mechanism}

Allergic conjunctivitis results from a Type 1 (immediate) immunoglobulin $\mathrm{E}$ (IgE)-mediated hypersensitivity reaction. The allergen particles bind to and cross-link $\operatorname{IgE}$ molecules that are attached to tissue mast cells, resulting in mast cell degranulation and release of histamines and other inflammatory mediators into the tissue. Histamine is the primary contributor to the development of early-phase signs and symptoms of SAC (Abelson 1979). A comprehensive summary of these is provided in Hingorani 1997.

\section{Clinical presentation}

Allergic conjunctivitis is usually bilateral. The main defining feature of this type of conjunctivitis is intense itching. More than 75\% of patients report this symptom when seeking treatment (Whitcup 2006). Other signs and symptoms include epiphora, hyperaemia (engorgement of blood vessels of the conjunctiva), micropapillae (little bumps on the inner surface of the eyelid), conjunctival chemosis (swelling of the conjunctiva), and thin mucous discharge.

If accompanied by rhinitis (sneezing, rhinorrhoea (runny nose), nasal itch or blockage), the symptom complex is sometimes referred to as rhinoconjunctivitis. Coughing and wheezing due to asthma may also co-exist. Diagnosis is usually on the basis of a careful allergy history and examination of the conjunctiva. Skinprick tests, serum-specific IgE, and conjunctival allergen challenge may be used to confirm the allergic trigger.

\section{Description of the intervention}

A variety of drugs have been used to treat allergic conjunctivitis, which can be administered orally or applied directly to the eye. Systemic treatment with oral antihistamines is usually indicated when other symptoms are being treated along with the conjunctivitis, such as rhinitis. Topical treatments include antihistamines, mast cell stabilisers, non-steroidal anti-inflammatory drugs, and corticosteroids, the last being reserved for severe symptoms that 
are unresponsive to other treatments and given for a short period of time.

\section{How the intervention might work}

The pharmacological effects of antihistamines are based on their abilities to block histamine $\mathrm{H} 1$ receptors. $\mathrm{H} 1$ receptors are activated by histamine, which has many actions. Histamine mediates the tissue response to injury (for example mechanical, thermal, infections, etc.). It is also a mediator of gastric acid secretion and may serve as a neurotransmitter. With respect to conjunctivitis, the action of antihistamines is to antagonise the vasoconstrictor, and, to a lesser extent, the vasodilator effects of histamine. Some antihistamines have central effects like sedation, which can influence their use when administered systemically.

Mast cell stabilisers inhibit degranulation by interrupting the normal chain of intracellular signals resulting from the cross-linking and activation of FceRI by allergen (Cook 2002). They inhibit mast cell degranulation, the release of histamine, and the other preformed mediators and the arachidonic acid cascade (Leonardi 2005).

\section{Why it is important to do this review}

As allergic conjunctivitis is considered to be a leading cause of acute red eye presentation to hospital, optometrists, and general practitioners, it has a significant impact on health service provision. The spectrum of disease varies from mild to severe and can therefore interfere with quality of life. It also has the potential to compromise visual function, although this is rarely seen.

Standard treatment is topical antihistamines alone or in combination with topical mast cell stabilisers. There is clinical uncertainty about the relative efficacy of this treatment.

\section{O B J E C T I VES}

The objective of this review was to assess the effects of topical antihistamines and mast cell stabilisers, alone or in combination, for use in treating seasonal and perennial allergic conjunctivitis.

\section{METHODS}

\section{Criteria for considering studies for this review}

\section{Types of studies}

We included randomised controlled trials (RCTs) only. We excluded within-person trials (in which eyes were randomly allocated to different treatments).

\section{Types of participants}

We included trials that recruited children or adults with seasonal allergic conjunctivitis (SAC) or perennial allergic conjunctivitis (PAC). This review did not cover other allergic conjunctivitis entities such as vernal keratoconjunctivitis, atopic keratoconjunctivitis, and giant papillary conjunctivitis. We attempted to obtain separate data for participants with SAC or PAC where trials also included participants with other types of conjunctivitis.

The diagnosis was done clinically based on the presence of typical symptoms and signs.

We excluded trials conducted with the use of conjunctival allergen challenge or conjunctival provocation testing, as it would be difficult to compare the data with trials that have participants who were recruited with active symptomatic disease without provocation tests. In addition, information from trials using provocation or challenge might not be representative and generalisable of the condition observed in routine practice.

We excluded people with allergic eye disease associated with atopy.

\section{Types of interventions}

This review included trials comparing topical antihistamines and mast cell stabilisers, alone or in combination, with placebo, no treatment, or any other antihistamine and/or mast cell stabiliser. These included the medications listed in Appendix 1 and any other pure antihistamine alone or in combination with a mast cell stabiliser not originally listed.

We placed no restriction on the duration of treatment in the trials. We did not include any trials that used herbal, oral, nasal antihistamines and any other medication.

\section{Types of outcome measures}

\section{Primary outcomes}

The primary outcome was any participant-reported evaluation (by questionnaire) of severity of four main ocular symptoms: itching, irritation, watering eye, and photophobia (dislike of light), both separately and, if possible, by an overall symptom score. We considered any follow-up time between one week and one year.

As there are a variety of different questionnaires evaluating ocular symptoms, we tried to transform data to common measurements where possible to facilitate meta-analysis using the standardised mean difference. We used this only if studies utilised different participant-reported outcomes measuring a comparable range of symptoms. 


\section{Secondary outcomes}

Secondary outcomes were measured at any reported follow-up time and included:

- Adverse events.

- Signs of hyperaemia and redness, chemosis, tarsal papillae (little bumps on inner surface of eyelid). Signs were clinician/ investigator assessed and analysed using categorisation where appropriate.

- Duration of symptoms (days) of acute episodes.

- Incidence of acute episodes (per year).

A variety of numeric rating scales were used to assess participantand clinician-reported symptoms in the included studies. Unless stated otherwise, higher scores represent higher levels of symptoms than lower scores.

\section{Search methods for identification of studies}

\section{Electronic searches}

We searched CENTRAL (which contains the Cochrane Eyes and Vision Trials Register) (2014, Issue 7), Ovid MEDLINE, Ovid MEDLINE In-Process and Other Non-Indexed Citations, Ovid MEDLINE Daily, Ovid OLDMEDLINE (January 1946 to July 2014), EMBASE (January 1980 to July 2014), the metaRegister of Controlled Trials ( $m$ RCT) (www.controlled-trials.com), ClinicalTrials.gov (www.clinicaltrials.gov) and the World Health Organization (WHO) International Clinical Trials Registry Platform (ICTRP) (www.who.int/ictrp/search/en). We did not use any date or language restrictions in the electronic searches for trials. We last searched the electronic databases on 17 July 2014.

See: Appendices for details of search strategies for CENTRAL (Appendix 2), MEDLINE (Appendix 3), EMBASE (Appendix 4), mRCT (Appendix 5), ClinicalTrials.gov (Appendix 6), and the ICTRP (Appendix 7).

\section{Searching other resources}

We searched the reference lists of review articles, book chapters, and relevant trial reports for details of further relevant publications. We planned to contact authors for data queries. We used the Science Citation Index to search for references that cite the studies that were included in the review.

\section{Data collection and analysis}

\section{Selection of studies}

Two review authors (MSM, MC/MZM) independently assessed the titles and abstracts resulting from the manual and electronic searches for selection of studies for inclusion. We obtained full copies of all relevant or potentially relevant trials and assessed these according to the 'Criteria for considering studies for this review' section. The investigators were not masked to the names of authors, institutions, journal of publication, or results when they made their assessments. We resolved disagreements about whether a trial should be included by discussion with a third review author $(\mathrm{AAB})$ and consensus. In cases where we needed additional information before we could make a decision about whether or not to include a trial, we planned to obtain this information by contacting the trial authors when author contact details were available. We attempted to obtain further information about any trial that had been published only as an abstract by contacting authors if contact details were available. If a full report was not available, we planned to assess and include data from abstracts for the review and meta-analysis. If there was not sufficient abstract data for the meta-analysis, we still planned to present the abstract data in the review to ensure no significant information was lost.

Using a form developed to document the process, we divided the full copies into two groups: 1) definitely include and 2) definitely exclude.

Agreement between the two review authors was recorded. At this stage, we excluded only those papers that both review authors had assigned to the 'definitely exclude' group. We documented and reported these exclusions in the review. We assessed all other papers for methodological quality.

\section{Data extraction and management}

As well as recording information about the methods used in the trial, we extracted the following information from the trial reports using a standardised form:

- details of participants (age, gender, setting, number in each group, comparability at baseline);

- details of interventions (dosage, schedule, compliance, comparison group, timing);

- outcomes (primary and secondary outcomes, adverse effects);

- other information (source of funding, declaration of interest).

Two review authors (MSM, MC/MZM) independently extracted the data for the primary and secondary outcomes on to a standardised form. One review author (MC) entered data into RevMan (RevMan 2014), and a second review author (AAB) checked the data entered into RevMan to ensure that no mistakes had been made. The review authors resolved any differences by discussion. If there was any doubt about the data of a trial, the review authors contacted the authors of the trial. Where studies were reported in more than one publication, we extracted data from each report separately. We then collated the information from the multiple data collection forms. 


\section{Assessment of risk of bias in included studies}

Two review authors (MC, $\mathrm{AAB}$ ) evaluated the risk of bias independently. They met after completion to identify disagreements. Review authors assessed trial quality according to the methods set out in Chapter 8 of the Cochrane Handbook for Systematic Reviews of Interventions (Higgins 2011a). We used Cochrane's 'Risk of bias tool' and considered six domains: sequence generation (randomisation), allocation concealment, blinding (masking), incomplete outcome data (completeness of follow-up), selective outcome reporting, and free from other bias.

Two review authors (MC, $\mathrm{AAB}$ ) assessed the risk of bias for each parameter and judged each parameter as low risk of bias, high risk of bias, or unclear (uncertain risk of bias where there was insufficient information to be able to judge).

We planned to perform a sensitivity analysis, if possible, excluding studies with high risk of bias to determine the effect of this decision on the results (Higgins 2011b).

\section{Measures of treatment effect}

\section{Dichomotous data}

For dichotomous data, we calculated odds ratios and their $95 \%$ confidence intervals.

\section{Continuous data}

For continuous data presented using a common scale, we calculated the mean difference and its $95 \%$ confidence interval. For overall symptom scores, we used the standardised mean difference was used.

\section{Unit of analysis issues}

The unit of randomisation was the individual participant (not eye) due to potential systemic absorption and effect in the fellow eye; we therefore only included studies that used the participant as the unit of randomisation. We considered cross-over trials if there was an adequate washout ( 48 hours) between the two treatment periods and if the treatment was compared with a control placebo. H1 antihistamine terminal elimination half-life values range from 2 hours for acrivastine to 27 hours for desloratadine (Simons 2002).

\section{Dealing with missing data}

If data were not obtainable we considered the potential impact of the missing data on the results and highlighted the potential impact in the 'Discussion' section of the review.

\section{Assessment of heterogeneity}

Before combining studies we planned to assess clinical heterogeneity by examination of the study details and statistical heterogeneity using the $\mathrm{Chi}^{2}$ test and the $\mathrm{I}^{2}$ statistic. Where we were unable to pool results we planned to provide a descriptive summary.

\section{Assessment of reporting biases}

We minimised reporting bias by maximising our search strategy to include research that had been published in different languages as well as unpublished research.

If appropriate, we planned to investigate publication bias by looking at a funnel plot of the data.

\section{Data synthesis}

When there were sufficient trials available without substantial heterogeneity, we combined studies in a meta-analysis using a random-effects model. Where this was not possible, we performed a narrative synthesis. As we evaluated a network of treatments, we also planned a network meta-analysis, provided suitable data were available.

\section{Subgroup analysis and investigation of heterogeneity}

Not applicable for this review.

\section{Sensitivity analysis}

We planned to perform sensitivity analyses, if possible, to assess how robust the results were to changes in methods such as:

1. excluding studies of lower methodological quality;

2. excluding unpublished studies;

3. excluding studies that assumed that eyes within a participant are independent.

\section{RES U L T S}

\section{Description of studies}

We have described the studies within the context of each comparison (see 'Effects of interventions' section).

\section{Results of the search}

The electronic searches yielded a total of 2764 references (Figure 1). The Trials Search Co-ordinator removed 1030 duplicate records, screened 1734 records, and removed 630 references that 
were not relevant to the scope of this review. We screened the remaining 1104 references and discarded 1054 reports as not relevant. We obtained 50 full-text reports for potential inclusion in the review and included 30 studies (see 'Characteristics of included studies' section) and excluded 17 studies (see 'Characteristics of excluded studies' section). Currently three studies are still awaiting classification: Scandashree 2013 has insufficient details on outcome measures, and Jia 2012 and Dharmistha 2013 have insufficient information on methods of allocation. If we are able to obtain further information on these studies, we will assess them in future updates of this review. 
Figure I. Results of searching for studies for inclusion in the review 2764 records identified through electronic database searching

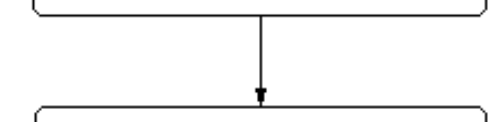

1734 records after duplicates removed

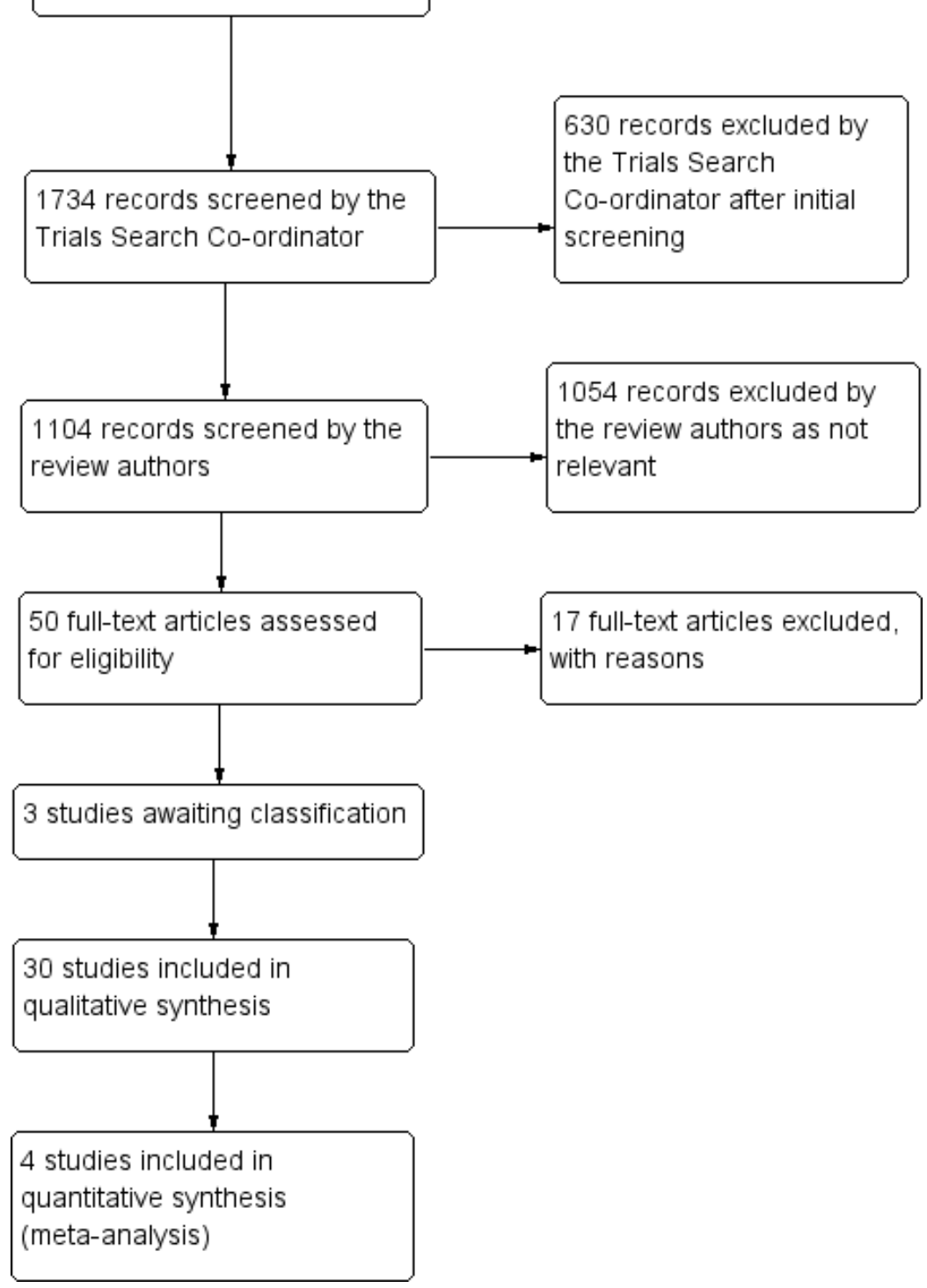




\section{Included studies}

We included 30 trials with 17 different comparisons (see Figure 2 , a network diagram showing the number of studies contributing to each of the 17 comparisons).

Figure 2. Network diagram: Number of studies by treatment comparison

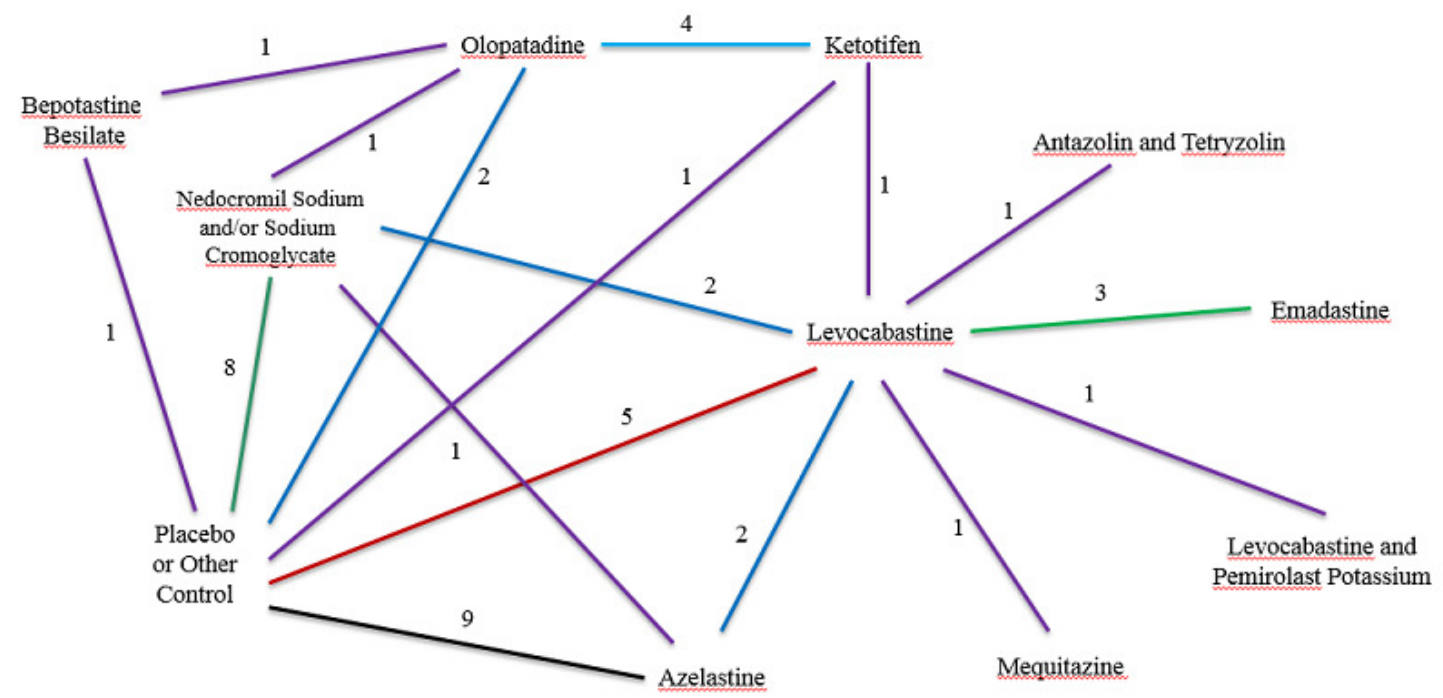

\section{Excluded studies}

We excluded 17 studies that did not meet the inclusion criteria (see 'Characteristics of excluded studies' section).

\section{Risk of bias in included studies}

We summarised risk of bias in Figure 3 and Figure 4. Most studies had low risk of bias regarding the masking of participants and investigators and selective reporting. Information on sequence generation and allocation concealment was frequently missing. 
Figure 3. 'Risk of bias' summary: review authors' judgements about each 'Risk of bias' item for each included study.

\begin{tabular}{|c|c|c|c|c|c|c|c|}
\hline & 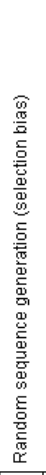 & 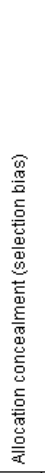 & 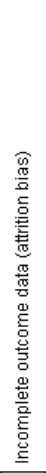 & 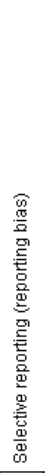 & 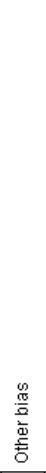 & 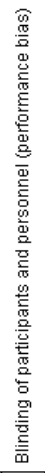 & 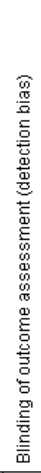 \\
\hline Avunduk 2005 & + & $?$ & $\odot$ & + & + & + & 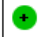 \\
\hline Azevedo 1991 & 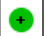 & $?$ & $\odot$ & $\odot$ & $\odot$ & $\odot$ & $\odot$ \\
\hline Canonica 2003 & $\odot$ & $?$ & $\odot$ & 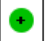 & 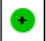 & $\odot$ & $\odot$ \\
\hline Carr 2013 & $\odot$ & $?$ & + & + & $\odot$ & $\odot$ & † \\
\hline Davies 1993 & $?$ & $?$ & $\odot$ & $\odot$ & $?$ & + & + \\
\hline Fujishima 2008 & $\odot$ & $?$ & $\odot$ & $\odot$ & $\odot$ & $?$ & $?$ \\
\hline Giede-Tuch 1998 & $?$ & $?$ & + & + & $\odot$ & + & + \\
\hline Graue 1994 & $?$ & $?$ & + & $\odot$ & $\odot$ & $\odot$ & 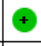 \\
\hline Hechanova 1984 & $?$ & $?$ & $\odot$ & $\odot$ & $\odot$ & $\odot$ & $\odot$ \\
\hline Höffling-Lima 2001 & + & $?$ & + & + & + & + & + \\
\hline James 2003 & $?$ & $?$ & $\odot$ & $\odot$ & $\odot$ & $?$ & $?$ \\
\hline Katelaris 2002 & + & $?$ & $?$ & $\odot$ & $\odot$ & $\odot$ & $\bullet$ \\
\hline Kidd 2003 & + & $?$ & $?$ & $\odot$ & $\odot$ & $\odot$ & $\odot$ \\
\hline Lanier 2001 & $?$ & $?$ & $?$ & $\odot$ & $\odot$ & $\odot$ & $\theta$ \\
\hline Leino 1992 & $?$ & $\odot$ & $\odot$ & $\odot$ & $\odot$ & $\odot$ & $\odot$ \\
\hline Lenhard 1997 & $?$ & $?$ & $?$ & + & $\odot$ & $?$ & $?$ \\
\hline McCabe 2012 & + & $?$ & + & + & $\odot$ & $\odot$ & $\theta$ \\
\hline Melamed 1994 & $?$ & $?$ & † & $\odot$ & $\odot$ & $\odot$ & $\odot$ \\
\hline Melamed 2000 & $?$ & $?$ & 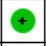 & + & + & $\odot$ & + \\
\hline Möller 1994 & $\odot$ & $\odot$ & $\odot$ & + & $\odot$ & $\odot$ & $\odot$ \\
\hline Nazarov 2003 & $?$ & $?$ & + & $\bullet$ & $\odot$ & $\odot$ & $\bullet$ \\
\hline Petzold 2002 & $?$ & $?$ & $?$ & $?$ & $?$ & $?$ & $?$ \\
\hline Sabbah 1998 & $?$ & $?$ & $\odot$ & $\odot$ & 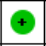 & $?$ & $?$ \\
\hline Sarker 2011 & $\odot$ & $?$ & $\oplus$ & 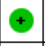 & $\odot$ & $\odot$ & $\odot$ \\
\hline Secchi 2000a & $?$ & $?$ & $\odot$ & $\odot$ & $\odot$ & + & $\odot$ \\
\hline Secchi 2000b & $?$ & $?$ & + & $?$ & $\odot$ & $\odot$ & + \\
\hline Trinquand 1999 & $?$ & $?$ & $?$ & $?$ & $?$ & $?$ & $?$ \\
\hline Varguez-Rodriguez 2009 & $?$ & $?$ & $\odot$ & + & $\odot$ & $\odot$ & + \\
\hline Verin 2001 & $?$ & $?$ & $?$ & $\odot$ & $\odot$ & $\odot$ & + \\
\hline Wertheimer 1997 & + & $?$ & $?$ & $\odot$ & $\odot$ & $?$ & $?$ \\
\hline
\end{tabular}


Figure 4. 'Risk of bias' graph: review authors' judgements about each 'Risk of bias' item presented as percentages across all included studies.

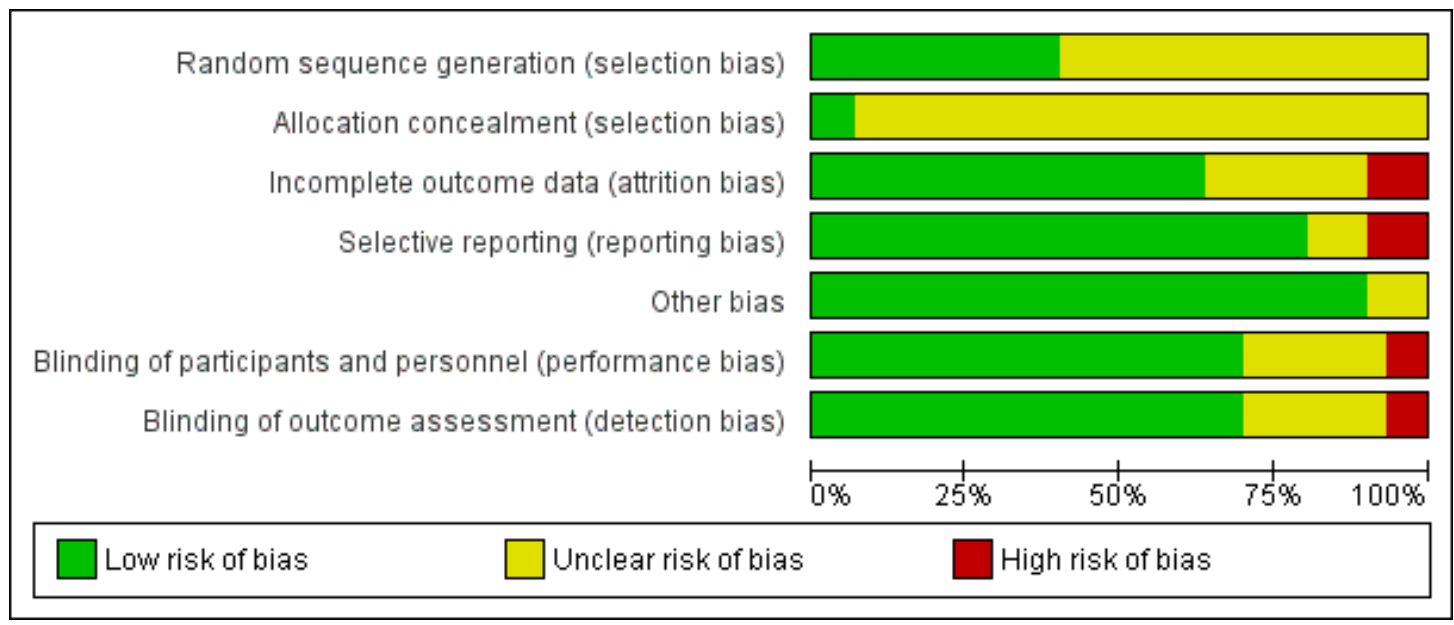

\section{Allocation}

We judged the method of randomisation to be at low risk of selection bias in approximately $37 \%$ of the studies (Figure 3).

Allocation concealment was the item with the lowest proportion (less than 10\%) of studies judged to be at low risk of bias. We judged more than $90 \%$ of studies as at unclear risk of selection bias on the basis of inadequate or no information provided on the method of allocation concealment.

\section{Blinding}

We judged the majority (approximately 62\%) of studies as at low risk of detection bias (masking of outcome assessment) and considered a similar proportion as at low risk of performance bias (masking of participants and investigators) (Figure 3).

\section{Incomplete outcome data}

Over $50 \%$ of the studies have low risk of bias for this domain. We judged a lower proportion of studies (approximately 36\%) with unclear risk of bias, as some of the trials did not conduct an intention-to-treat analysis, or no reasons for dropouts were described (Figure 3).

\section{Selective reporting}

We judged a highest proportion of studies (75\%) with low risk of bias for this domain.

\section{Other potential sources of bias}

Not applicable for this review.

\section{Effects of interventions}

\section{Drug comparisons}

We have used the following order to describe the comparisons: placebo-controlled studies with mast cell stabilisers (comparison 1); placebo-controlled studies with antihistamines (comparisons 2 and 3 , in order of number of studies available); and comparative studies of antihistamines and/or mast cell stabilisers (comparisons 4 to 16 , in order of number of studies available). We described primary and safety outcomes for each comparison. Secondary outcomes were either not reported (duration of symptoms of acute episodes and incididence of acute episodes) or uncommonly reported and using different measures and scales (signs evaluated by an investigator) and thus were not analysed. 


\section{Nedocromil sodium/sodium cromoglycate versus placebo}

The search identified eight eligible studies comparing the mast cell stabilisers nedocromil sodium or sodium cromoglycate against placebo (Azevedo 1991; Davies 1993; Hechanova 1984; James 2003; Leino 1992; Melamed 1994; Melamed 2000; Möller 1994). One study was a three-arm trial comparing both nedocromil sodium and sodium cromoglycate with placebo (Leino 1992). Of the remaining seven studies, four examined sodium cromoglycate (Azevedo 1991; Davies 1993; Hechanova 1984; James 2003), and two examined nedocromil sodium (Melamed 2000; Möller 1994). Three studies also included a third treatment arm: either levocabastine, in Azevedo 1991 and Davies 1993, or azelastine (James 2003). One study was reported in two publications (Melamed 1994; Melamed 2000); Melamed 2000 reported combined results from two separate studies, one of which had been previously reported in Melamed 1994.

The number of participants randomised (or analysed if numbers randomised not known) to nedocromil sodium/sodium cromoglycate and placebo were 21 versus 21 (Azevedo 1991), 32 versus 32 (Davies 1993), 20 versus 20 (Hechanova 1984), 50 versus 49 (James 2003), 61 versus 64 (Leino 1992), 43 versus 43 (Melamed 1994), 94 versus 95 (Melamed 2000), and 77 versus 72 (Möller 1994), respectively.

Duration of treatment was two weeks in one study (James 2003), four weeks in five studies, and eight weeks in one study (Melamed 1994; Melamed 2000).

\section{Primary outcomes}

Although all eight studies reported at least one of the four symptoms prespecified as primary outcomes (itching, irritation, watering eyes, or photophobia), there was considerable variation in how these outcomes were reported (Table 1).

Six studies collected data on itching. Three of these studies collected participant-reported itching scores using a 0-4 scale, however as no studies also reported a standard deviation, we considered it not possible to perform formal meta-analysis. All studies did report less itching for the active treatment group compared with placebo. Hechanova 1984 presented mean participant-reported scores of 0.96 (sodium cromoglycate) versus 2.10 (placebo) at 14 days, with even larger differences in favour of the active treatment at 21 and 28 days. Melamed 1994 reported scores (it was unclear whether this was the mean or median) of 1.14 (nedocromil sodium) versus 1.48 (placebo) at 14 days. Melamed 2000 reported only change scores from baseline to the peak pollen period and found a statistically significant difference in favour of nedocromil sodium. Möller 1994 reported mean scores of 0.9 (nedocromil sodium) versus 1.4 (placebo) during peak pollen challenge in a graph. Leino 1992 reported that both the sodium cromoglycate and nedocromil sodium groups had statistically significantly less itching than the placebo group, but presented no data. James 2003 only reported itching as part of an overall symptom score plus the proportion of participants with improvement in itching in each group between days 0 and 3. Although Davies 1993 collected daily data on itch from participant diaries, this publication did not specifically report any data.

Three studies presented data for participant-reported ocular irritation (including grittiness and foreign body sensation), but overall the evidence for the effectiveness of the active treatment was limited. At 14 days, Hechanova 1984 reported mean grittiness scores of 0.58 (sodium cromoglycate) versus 1.31 (placebo) using a 0-4 scale, a difference that was considered statistically significant. Azevedo 1991 presented daily mean irritation using a 0-4 scale in a graph: at 14 days, mean irritation was around 1 for both groups. Between-group differences for the median area under the curve were also similar when the entire 28-day follow-up period was considered. In the study by Möller 1994, mean grittiness (estimated from a graph) during peak pollen challenge was 0.2 (nedocromil sodium) versus 0.5 (placebo). Leino 1992 reported statistically significant differences in favour of both treatment groups versus placebo when considering the change from baseline to four weeks, but not at one week; once again, no actual data were presented. James 2003 only reported foreign body sensation in terms of improvement from day 0 to day 3. Davies 1993 also collected information on ocular irritation, but again did not report this information directly.

Data on watering eyes was not commonly reported. Using a 0-4 scale, Melamed 1994 reported scores of 0.71 (nedocromil sodium) versus 1.08 (placebo) at 14 days, but it was unclear whether these were mean values. In the later publication by this group (Melamed 2000), both treatment groups in fact experienced an increase in tearing during the peak pollen period. Using a 0-4 scale, Möller 1994 reported mean watering scores of 0.4 (nedocromil sodium) versus 0.7 (placebo) during peak pollen challenge. Azevedo 1991 only reported changes in tearing over 28 days, and again James 2003 reported improvement in tearing from baseline to day 3 . Davies 1993 and Leino 1992 did not report any information relating to this outcome, even though this had been collected using participant diaries.

Six studies were known to have collected data on photophobia, but there was no clear evidence available concerning this outcome. Two studies did not report any information on this outcome (Davies 1993; Leino 1992), and two studies reported change scores (Azevedo 1991; James 2003). Hechanova 1984 reported a statistically significant difference in favour of sodium cromoglycate, but only at the 14-day time point. The remaining study reported mean scores of around 0.3 on a $0-4$ scale for both the nedocromil sodium and placebo groups (Möller 1994).

\section{Overall symptom score}

Five articles representing four studies reported some kind of global evaluation or overall symptom score (Table 1). Melamed 2000 reported a participant-reported composite symptom score during 
the peak pollen period, and similar separate results for one of the two constituent substudies were also reported (Melamed 1994). James 2003 reported both investigator- and participant-reported composite symptom scores up to 14 days. Leino 1992 collected similar composite scores from investigators and participants but only reported that there were no statistically significant differences. Davies 1993 reported the participants' global evaluation of treatment efficacy using a 4-point scale. Although all available results from these studies tended to favour the active treatment, no standard deviations were reported and no formal meta-analysis was conducted.
No serious adverse events were observed. Overall, placebo, nedocromil sodium, and sodium cromoglycate were well tolerated.

\section{Summary}

There was some evidence from individual trials that nedocromil sodium or sodium cromoglycate is more effective than placebo in improving ocular symptoms. However, it was not possible to perform formal meta-analyses for this comparison due to variation in how outcomes were reported and the lack of suitable data (especially standard deviations (SDs)).

Table 1. Nedocromil sodium or sodium cromoglycate versus placebo. Outcome definition, time points, and summary of results

Safety

\begin{tabular}{|c|c|c|c|c|c|}
\hline Article & Outcome definition & Time point & $\begin{array}{l}\text { Nedocromil } \\
\text { sodium or sodium } \\
\text { cromoglycate }\end{array}$ & Placebo & $\begin{array}{l}\text { Number of partic- } \\
\text { ipants randomised } \\
\text { (n) and comments }\end{array}$ \\
\hline Davies 1993 & $\begin{array}{l}\text { Partic- } \\
\text { ipants' global evalua- } \\
\text { tion of treatment ef- } \\
\text { ficacy (4-point scale) }\end{array}$ & 28 days & $\begin{array}{l}\text { Cromoglycate: } \\
\text { Excellent/good: } 68 \% \\
\text { Moderate/poor: } \\
32 \%\end{array}$ & $\begin{array}{l}\text { Excellent/good: } 63 \% \\
\text { Moderate/poor: } \\
37 \%\end{array}$ & $\begin{array}{l}\mathrm{n}=95 \\
\text { Individual categories } \\
\text { may be estimated } \\
\text { from graph, and } \\
\text { mean score could be } \\
\text { calculated if consid- } \\
\text { ered appropriate }\end{array}$ \\
\hline James 2003 & $\begin{array}{l}\text { Investigator's } \\
\text { composite symptom } \\
\text { score (itching, tear- } \\
\text { ing, conjuncti- } \\
\text { val redness) (range 0- } \\
\text { 9), participant's com- } \\
\text { posite } \\
\text { symptom score (itch- } \\
\text { ing, redness, tearing) } \\
\text { (range } 0-9 \text { ) }\end{array}$ & 14 days & $\begin{array}{l}\text { Cromoglycate: } \\
\text { Investigator: Mean } \\
2.2 \\
\text { Participant: Mean } 1 . \\
8\end{array}$ & $\begin{array}{l}\text { Investigator: Mean } \\
2.9 \\
\text { Participant: Mean } 2 . \\
8\end{array}$ & $\begin{array}{l}\mathrm{n}=144 \\
\text { Estimated from } \\
\text { graph (no SD). Days } \\
3 \text { and } 7 \text { also available } \\
\text { (investi- } \\
\text { gator-reported); days } \\
1-14 \text { available (par- } \\
\text { ticipant-reported) }\end{array}$ \\
\hline Leino 1992 & $\begin{array}{l}\text { Overall assess- } \\
\text { ment by participants } \\
\text { and investigators (4- } \\
\text { point scale) }\end{array}$ & 28 days & $\begin{array}{l}\text { Cromoglycate and } \\
\text { nedocromil: } \\
\text { No data presented }\end{array}$ & No data presented & $\begin{array}{l}\mathrm{n}=195 \\
\text { No significant dif- } \\
\text { ferences between the } \\
3 \text { groups (except for } \\
\text { subgroup analysis by } \\
\text { centre) }\end{array}$ \\
\hline Melamed 1994 & $\begin{array}{l}\text { Participant- } \\
\text { reported composite } \\
\text { symptom score: itchy }\end{array}$ & 14 days & $\begin{array}{l}\text { Nedocromil: } \\
\text { Mean } 3.8\end{array}$ & Mean 5.1 & $\begin{array}{l}\mathrm{n}=86 \\
\text { No SD. Estimated } \\
\text { from graph in Fig- }\end{array}$ \\
\hline
\end{tabular}

Topical antihistamines and mast cell stabilisers for treating seasonal and perennial allergic conjunctivitis (Review)

Copyright () 2015 The Cochrane Collaboration. Published by John Wiley \& Sons, Ltd. 


\begin{tabular}{|c|c|c|c|c|c|}
\hline & $\begin{array}{l}\text { eyes, burning eyes, } \\
\text { tearing eyes, overall } \\
\text { eye condition (range } \\
0-16 \text { ) }\end{array}$ & & & & $\begin{array}{l}\text { ure } 2 \text { of the orig- } \\
\text { inal report. Slightly } \\
\text { unclear which symp- } \\
\text { toms contributed to } \\
\text { this score }\end{array}$ \\
\hline Melamed 2000 & $\begin{array}{l}\text { Participant- } \\
\text { reported composite } \\
\text { symptom score: itchy } \\
\text { eyes, burning eyes, } \\
\text { tearing eyes, overall } \\
\text { eye condition (range } \\
0-16 \text { ) }\end{array}$ & "peak pollen period" & $\begin{array}{l}\text { Nedocromil: } \\
\text { Mean } 3.95\end{array}$ & Mean 4.92 & $\begin{array}{l}\mathrm{n}=189 \\
\text { No SD. The time } \\
\text { point varied by in- } \\
\text { cluded study. One } \\
\text { of the two studies } \\
\text { is Melamed 1994, } \\
\text { above }\end{array}$ \\
\hline
\end{tabular}

\section{Footnotes}

SD: standard deviation

\section{Azelastine versus placebo}

The search strategy identified nine eligible studies comparing the antihistamine azelastine against placebo (Canonica 2003; Giede-Tuch 1998; James 2003; Lenhard 1997; Nazarov 2003; Petzold 2002; Sabbah 1998). Petzold 2002 summarised data from three different RCTs, which we analysed as individual studies. Three studies reported a third treatment arm, comparing azelastine against levocabastine, in Canonica 2003 and Sabbah 1998, and azelastine against sodium cromoglycate (James 2003). All studies were available as full- text papers, except for Petzold 2002, which was available only as an abstract. Drug concentration was the same in all studies (azelastine 0.05\%), however two studies compared two different concentrations (azelastine $0.05 \%$ and $0.025 \%$ ) (Giede-Tuch 1998; Lenhard 1997). Azelastine was administered twice a day.

The number of participants randomised (or analysed if numbers randomised not known) to azelastine and placebo were 57 versus 56 (Canonica 2003), 99 versus 52 (Giede-Tuch 1998), 45 versus 49 (James 2003), 92 versus 94 (Lenhard 1997), 58 versus 58 (Nazarov 2003), 160 versus 80 (Petzold 2002), 99 versus 46 (Petzold 2002), 49 versus 29 (Petzold 2002), and 51 versus 30 (Sabbah 1998), respectively

Duration of treatment was six weeks in three studies (Canonica 2003; Nazarov 2003; Sabbah 1998), two or four weeks in three trials reported by Petzold 2002, and two weeks in three other studies (Giede-Tuch 1998; James 2003; Lenhard 1997).

\section{Primary outcomes}

Although all nine studies reported at least one of the four prespecified primary outcomes (itching, irritation, watering eyes, or photophobia), there were some variations in how these outcomes were reported across the included studies.

Data on itching were collected as mean scores in five studies using a 0-3 scale, where higher scores represented worse itching, but as SD was not reported, meta-analysis was not feasible (Giede-Tuch 1998; James 2003; Lenhard 1997; Nazarov 2003; Sabbah 1998). All studies reported less itching with azelastine compared to placebo, but in some studies it was not possible to confirm whether the results were statistically significant (Lenhard 1997). Giede-Tuch 1998 presented participant-reported mean scores of 0.75 (azelastine $0.05 \%$ ) and 0.90 (azelastine $0.025 \%$ ) versus 1.15 (placebo) at 14 days. SD was not available. Petzold 2002 summarised data from three RCTs assessing investigators' score on severity of itching; one of these three trials (study ID 3021), with mean scores of 0.53 (azelastine) versus 1.39 (placebo), had statistically significant results in favour of the active treatment. The differences reported in the other two RCTs were not statistically significant, that is study ID 3062 reported mean scores 0.73 (azelastine) and 0.76 (placebo), and study ID 3034 reported mean scores 0.76 (azelastine) and 1.10 (placebo).

Four studies presented data for watering eyes or tearing using a 0-3 scale or sum scores of symptoms (Giede-Tuch 1998; James 2003; Lenhard 1997; Sabbah 1998). Giede-Tuch 1998 presented participant-reported tearing as mean scores (data estimated from graph) of 0.45 and 0.35 (azelastine $0.05 \%$ and $0.025 \%$, respectively) versus 0.55 (placebo) at 14 days. No SD was reported. Sabbah 1998 used response rates (calculated from participants' diaries), and Lenhard 1997 reported participants' tearing scores using the same 0-3 scale as for itching.

Data on ocular irritation (described as foreign body sensation) and photophobia were not frequently reported. In James 2003, there was improvement in both study and control groups, although the results were not statistically significant. Some studies reported ocu- 
lar irritation and photophobia as part of a composite investigatorreported sum score, for example Sabbah 1998, in which foreign body sensation was part of an 8-symptom sum score at days 3 and 14 .

\section{Overall symptom scores}

One study, Sabbah 1998, reported itching as composite sum score and response rates on three eye symptoms (itching, tearing, and conjunctival redness), calculated from participants' diaries. Two studies reported participant composite sum score based on two symptoms (itching and redness) using sum mean score (0-6 scale) (Canonica 2003; Nazarov 2003), however neither of these studies reported SD. Nazarov 2003 reported sum score of 1.9 (azelastine) versus 3.0 (placebo), estimated from a published graph at day 14 and the differences consistently increased in favour of the active treatment with longer follow-up, until day 42 . These results were consistent with investigators' assessment reported as statistically significant. Canonica 2003 reported investigators' sum scores on itching and redness with mean scores and SD of 1.8 (1.4) (azelas- tine) versus 3.1 (1.5) (placebo), with change at day 7 statistically significant.

\section{Safety outcomes}

No serious adverse events or changes in vital signs were reported during the treatment. Overall, azelastine and placebo were well tolerated by participants across all the studies; few cases withdrew from azelastine or placebo groups due to insufficient tolerability (burning sensation or bitter taste, or both).

\section{Summary}

There was some evidence from individual studies that azelastine improved some symptoms more than placebo. However, it was not possible to perform formal meta-analyses for this treatment comparison due to variations of outcomes reported and the lack of suitable data.

Table 2. Azelastine versus placebo. Outcome definition, time points, and summary of results

\begin{tabular}{|c|c|c|c|c|c|}
\hline Article & $\begin{array}{l}\text { Outcome } \\
\text { definition }\end{array}$ & Time point & Azelastine & Placebo & $\begin{array}{l}\text { Number of partici- } \\
\text { pants randomised } \\
\text { (n) and comment }\end{array}$ \\
\hline Canonica 2003 & $\begin{array}{l}\text { Investigator's assess- } \\
\text { ment } \\
\text { of change in clinical } \\
\text { sum score (itching, } \\
\text { redness) (range } 0-6 \text { ) }\end{array}$ & 7 days & $\begin{array}{l}\text { Investigator: day } 7 \\
\text { Mean sum scores } \\
\text { (SD) } \\
1.8(1.4) \\
\text { Participants: } \\
\text { Mean score (itching } \\
\text { and conjunctiva red- } \\
\text { ness) } 1.65 \text { at day } 14\end{array}$ & $\begin{array}{l}\text { Investigator: day } 7 \\
\text { Mean sum scores } \\
\text { (SD) } \\
3.1 \text { (1.5) } \\
\text { Participants: } \\
\text { Mean score (itching } \\
\text { and c. redness) } 3.85 \text { at } \\
\text { day } 14\end{array}$ & $\begin{array}{l}\mathrm{n}=139 \\
\text { Main variable: Time } \\
\text { course of sum score of } \\
\text { main eye symptoms at } \\
\text { day } 7 \\
\text { Secondary outcomes: } \\
\text { symptoms day 21, } 42\end{array}$ \\
\hline Giede-Tuch 1998 & $\begin{array}{l}\text { Partic- } \\
\text { ipants' (responders) } \\
\text { rate sum score (it- } \\
\text { ching, lacrimation, } \\
\text { red- } \\
\text { ness) decreased by at } \\
\text { least } 3 \text { score points } \\
\text { between day 0-3; In- } \\
\text { vestigators' compos- } \\
\text { ite symp- } \\
\text { tom mean score (it- } \\
\text { ching, lacrimation, } \\
\text { redness) }\end{array}$ & 14 days & $\begin{array}{l}\text { Participants' response } \\
\text { rate at day } 3(0.025 \% \\
\text { and } 0.05 \%) \text { : } \\
73 \% \text { and } 82 \% \text {, re- } \\
\text { spectively } \\
\text { Investigator: } \\
\text { Mean score } \\
\text { of three symptoms ( } 0 \text {. } \\
05 \% \text { dose) } 7 \text { days } \\
2.4\end{array}$ & $\begin{array}{l}\text { Participants' response } \\
\text { rate: } 56 \% \\
\text { Investigator: } \\
\text { Mean score of } 3 \text { symp- } \\
\text { toms, } 7 \text { days } \\
3.5\end{array}$ & $\begin{array}{l}\mathrm{n}=151 \\
\text { Investigators' mean } \\
\text { estimated from graph } \\
\text { (no SD) }\end{array}$ \\
\hline
\end{tabular}

Topical antihistamines and mast cell stabilisers for treating seasonal and perennial allergic conjunctivitis (Review) 
(Continued)

\begin{tabular}{|c|c|c|c|c|c|}
\hline James 2003 & $\begin{array}{l}\text { Investigator's com- } \\
\text { posite sum symp- } \\
\text { tom score (itching, } \\
\text { tearing, conjuncti- } \\
\text { val redness) (range } \\
0-9 \text { ), par- } \\
\text { ticipant's composite } \\
\text { symptom score (it- } \\
\text { ching, redness, tear- } \\
\text { ing) (range 0-9) }\end{array}$ & 14 days & $\begin{array}{ll}\text { Investigator: } & \text { Mean } \\
\text { score } 2.2 & \\
\text { Participant: } & \text { Mean } \\
\text { score } 1.9 & \end{array}$ & $\begin{array}{ll}\text { Investigator: } & \text { Mean } \\
\text { score } 2.9 & \\
\text { Participant: } & \text { Mean } \\
\text { score } 2.8 & \end{array}$ & $\begin{array}{l}\mathrm{n}=144 \\
\text { Mean } \\
\text { scores estimated from } \\
\text { the graphs (no SD) } \\
\text { Days } 3 \text { and } 7 \\
\text { also available (investi- } \\
\text { gator-reported); days } \\
\text { 1-14 available (partic- } \\
\text { ipant-reported) }\end{array}$ \\
\hline Lenhard 1997 & $\begin{array}{l}\text { Investigator's com- } \\
\text { posite sum symp- } \\
\text { tom score (itching, } \\
\text { tearing, conjuncti- } \\
\text { val redness) } \\
\text { (range } 0-9 \text { ) } \\
\text { (Secondary analysis) }\end{array}$ & 7 and 14 days & $\begin{array}{l}\text { In- } \\
\text { vestigator: Mean score } \\
\text { of } 3 \text { symptoms (itch- } \\
\text { ing, lacrimation, and } \\
\text { c. redness) } \\
2.0 \text { at day } 14\end{array}$ & $\begin{array}{l}\text { In- } \\
\text { vestigator: Mean score } \\
\text { of } 3 \text { symptoms (itch- } \\
\text { ing, lacrimation, and } \\
\text { c. redness) } \\
2.1 \text { at day } 14\end{array}$ & $\begin{array}{l}\mathrm{n}=278 \\
\text { Mean scores (no SD) } \\
\text { due to high variabil- } \\
\text { ity for itching and } \\
\text { marked placebo re- } \\
\text { sponse, a secondary, } \\
\text { more objective analy- } \\
\text { sis was performed } \\
\text { Participant-re- } \\
\text { ported itching, c. red- } \\
\text { ness, lacrimation, and } \\
\text { swollen eyelids on a } 0 \text { - } \\
3 \text { scale }\end{array}$ \\
\hline Nazarov 2003 & $\begin{array}{l}\text { Investiga- } \\
\text { tor's composite sum } \\
\text { symptom score (it- } \\
\text { ching, conjunctival } \\
\text { redness) on day } 7\end{array}$ & 7 days & $\begin{array}{l}\text { Investigator: sum } \\
\text { score } 1.9 \\
\text { day } 14 \text { (estimated } \\
\text { from graph) }\end{array}$ & $\begin{array}{l}\text { Investigator: sum } \\
\text { score } 3.0 \\
\text { day } 14 \text { (estimated } \\
\text { from graph) }\end{array}$ & $\begin{array}{l}\mathrm{n}=116 \\
\text { Secondary variables: } \\
\text { outcomes measured } \\
\text { days } 21,42 \\
\text { Participant-reported } \\
\text { symptoms: } \\
\text { used to corroborate } \\
\text { clinical assessments }\end{array}$ \\
\hline $\begin{array}{l}\text { Petzold } 2002 \\
\text { (ID 3021) }\end{array}$ & \multirow{3}{*}{$\begin{array}{l}\text { Investigator's } \\
\text { assessment of sever- } \\
\text { ity score for both (it- } \\
\text { ching, redness) and } \\
\text { separated for each } \\
\text { symptom (itching, } \\
\text { redness) (range 0-3) }\end{array}$} & \multirow[t]{3}{*}{3 and 14 days } & $\begin{array}{l}\text { Investigator: } \\
\text { Mean score day } 14 \\
0.53\end{array}$ & $\begin{array}{l}\text { Investigator: } \\
\text { Mean score day } 14 \\
1.39\end{array}$ & \multirow{3}{*}{$\begin{array}{l}\text { ID 3021: } \mathrm{n}=78 \\
\text { ID 3062: } \mathrm{n}=145 \\
\text { ID 3034: } \mathrm{n}=240 \\
\text { Treatment duration of } \\
14 \text { days ( } 2 \text { studies) or } \\
28 \text { days ( } 1 \text { study) }\end{array}$} \\
\hline $\begin{array}{l}\text { Petzold } 2002 \\
\text { (ID 3062) }\end{array}$ & & & $\begin{array}{l}\text { Investigator: } \\
\text { Mean score day } 14 \\
0.73\end{array}$ & $\begin{array}{l}\text { Investigator: } \\
\text { Mean score day } 14 \\
0.76\end{array}$ & \\
\hline $\begin{array}{l}\text { Petzold } 2002 \\
\text { (ID 3034) }\end{array}$ & & & $\begin{array}{l}\text { Investigator: } \\
\text { Mean score day } 14 \\
0.76\end{array}$ & $\begin{array}{l}\text { Investigator: } \\
\text { Mean score day } 14 \\
1.10\end{array}$ & \\
\hline Sabbah 1998 & $\begin{array}{l}\text { Investigator's re- } \\
\text { sponders rate based } \\
\text { on decrease of at }\end{array}$ & 3 and 14 days & $\begin{array}{l}\text { Investigator: } \\
\text { Mean sum scores } 1.85 \\
\text { (no SD) at day } 14\end{array}$ & $\begin{array}{l}\text { Investigator: } \\
\text { Mean sum scores } 2.45 \\
(\text { no SD) at day } 14\end{array}$ & $\begin{array}{l}\mathrm{n}=113 \\
\text { Investigators' mean } \\
\text { estimated from graph }\end{array}$ \\
\hline
\end{tabular}

Topical antihistamines and mast cell stabilisers for treating seasonal and perennial allergic conjunctivitis (Review) 


\begin{tabular}{|l|l|l|l}
\hline $\begin{array}{l}\text { least } 3 \text { points in the } \\
\text { sum symptom score } \\
\text { (itching, conjuncti- }\end{array}$ & $\begin{array}{l}\text { Investigator: } \\
\text { Difference in the } \\
\text { mean sum }\end{array}$ & $\begin{array}{l}\text { Investigator: } \\
\text { Difference in the Participant-reported } \\
\text { mean sum }\end{array}$ \\
$\begin{array}{l}\text { val redness, lacrima- } \\
\text { tion) (range 0-9) be- } \\
\text { tween day 0 to 3; } \\
\text { par- } \\
\text { ticipant's composite } \\
\text { sum symptom score } \\
\text { (itching, conjuncti- } \\
\text { val redness, lacrima- } \\
\text { tion) (range 0-9) }\end{array}$ & $\begin{array}{l}\text { junctival redness, and junctival redness, and sum symptom score } \\
\text { lacrimation) }\end{array}$ \\
\hline
\end{tabular}

Footnotes

SD: standard deviation

\section{Levocabastine versus placebo}

The search strategy identified five eligible studies comparing the antihistamine levocabastine versus placebo (Azevedo 1991; Canonica 2003; Davies 1993; Graue 1994; Sabbah 1998). Four of these studies included a third arm comparing levocabastine against azelastine, in Canonica 2003 and Sabbah 1998, and levocabastine against nedocromil sodium or sodium cromoglycate (Azevedo 1991; Davies 1993). Drug concentration was the same in all the studies, $0.05 \%$ or $0.5 \mathrm{mg} / \mathrm{ml}$ instilled two to four times a day, but Canonica 2003 did not specify dose or concentrations.

The number of participants randomised (or analysed if numbers randomised not known) to levocabastine and placebo were 18 versus 21 (Azevedo 1991), 26 versus 52 (Canonica 2003), 31 versus 32 (Davies 1993), 20 versus 20 (Graue 1994), and 32 versus 30 (Sabbah 1998), respectively.

Duration of treatment was variable: six weeks in two studies ( Canonica 2003; Sabbah 1998), four weeks in two studies (Azevedo 1991; Davies 1993), and one week in one study (Graue 1994).

\section{Primary outcomes}

Data on itching were reported in four studies with some variations in how this symptom was reported. One study did not report itching as a main eye symptom but reported the other three primary outcomes (Azevedo 1991). Grass pollen counts were counted daily during the treatment period of some studies (Azevedo 1991; Davies 1993). Graue 1994 reported only the percentages in various severity categories using graphs. There were no statistically significant differences between the levocabastine and placebo groups $(\mathrm{P}=0.45)$ for improvement of itching at 7 days.

Azevedo 1991 collected data on participant-reported ocular irrita- tion as percentage of days with absence of ocular irritation. Sixtythree percent had symptom-free days in the levocabastine group versus $44 \%$ after placebo $(\mathrm{P}<0.06)$. Itching was also reported in a graph using median area under the curve: $14 \%$ (levocabastine) and 29\% (placebo) (data estimated from graph). Graue 1994 reported ocular irritation (foreign body sensation), but there was no statistically significant difference between groups $(\mathrm{P}=0.178)$.

Azevedo 1991 reported tearing as the percentage of days free of tearing, reporting larger differences during peak pollen days, with tearing absent in $88 \%$ (levocabastine) of the days under treatment versus 58\% (placebo) $(\mathrm{P}=0.01)$. Graue 1994 also reported statistically significant results with improvement of tearing scores $(\mathrm{P}=$ 0.006).

Data on photophobia were collected and reported as individual symptom or as composite sum score. Graue 1994 reported statistically significant results $(P=0.06)$ in favour of levocabastine compared with placebo.

\section{Overall symptom scores}

Some studies reported sum scores including itching (Canonica 2003; Sabbah 1998). Canonica 2003 used the mean score for itching and conjunctival redness (0-6 scale) with mean scores 1.5 (levocabastine) versus 3.85 (placebo) (estimated from a graph), but SD was not given. Davies 1993 reported percentages of global efficacy of treatment with significant participant-reported grading in which participants considered treatment as excellent or good in $87 \%$ (levocabastine) against $63 \%$ (placebo) $(\mathrm{P}=0.05)$; similar results were observed from investigators' assessed overall score of efficacy of treatment ( $91 \%$ levocabastine versus $68 \%$ placebo).

\section{Safety outcomes}


Overall, levocabastine and placebo were well tolerated by participants across the studies with no reports of serious adverse events. No difference was reported in the incidence of adverse events between the active and placebo groups, however one study reported higher incidence of (mild) adverse events in the placebo group compared to the levocabastine group (Graue 1994).
There was some evidence from individual studies that levocabastine-treated participants had better outcomes than those who received placebo. However, it was not possible to perform formal meta-analyses for this comparison due to variations of outcomes reported and lack of suitable data.

Table 3. Levocabastine versus placebo. Outcome definition, time points, and summary of results

Summary

\begin{tabular}{|c|c|c|c|c|c|}
\hline Article & Outcome definition & Time point & Levocabastine & Placebo & $\begin{array}{l}\text { Number of partici- } \\
\text { pants randomised (n) } \\
\text { and comment }\end{array}$ \\
\hline Azevedo 1991 & $\begin{array}{l}\text { Investigator's assess- } \\
\text { ment sum score (oc- } \\
\text { ular irritation, c. red- } \\
\text { ness, photopho- } \\
\text { bia, tearing, swollen } \\
\text { eyelids, and conjunc- } \\
\text { tival oedema) (range } \\
0-3 \text { ); participant-re- } \\
\text { ported same symp- } \\
\text { toms using VAS scale }\end{array}$ & 14 and 28 days & $\begin{array}{l}\text { Investigators: } \\
\text { Mean severity scores } \\
\text { for each symptom (ir- } \\
\text { rita- } \\
\text { tion, tearing, and pho- } \\
\text { tophobia) (data not re- } \\
\text { ported, only change } \\
\text { score from baseline) } \\
\text { Participants: } \\
\text { median area under the } \\
\text { curve (\%) } \\
14 \%\end{array}$ & $\begin{array}{l}\text { Investigators: } \\
\text { Mean severity scores } \\
\text { for each symptom (ir- } \\
\text { rita- } \\
\text { tion, tearing, and pho- } \\
\text { tophobia) (data not re- } \\
\text { ported, only change } \\
\text { score from baseline) } \\
\text { Participants: } \\
\text { median area under the } \\
\text { curve (\%) } \\
29 \%\end{array}$ & $\begin{array}{l}\mathrm{n}=63 \\
\text { Investigators' and par- } \\
\text { ticipants' global assess- } \\
\text { ment of efficacy at the } \\
\text { end of treatment }\end{array}$ \\
\hline Canonica 2003 & $\begin{array}{l}\text { Investigator's assess- } \\
\text { ment of change in } \\
\text { clinical sum score (it- } \\
\text { ching, redness) } \\
\text { (range 0-6) }\end{array}$ & 7 days & $\begin{array}{l}\text { Investigator: day } 7 \\
\text { Mean sum scores (SD) } \\
2.2(1.2) \\
\text { Participants: } \\
\text { Mean score } \\
1.5 \text { at day } 14 \text { (itching } \\
\text { and conjunctival red- } \\
\text { ness) }\end{array}$ & $\begin{array}{l}\text { Investigator: day } 7 \\
\text { Mean sum scores (SD) } \\
3.1 \text { (1.5) } \\
\text { Participants: } \\
\text { Mean score } 3.85 \text { at day } \\
14 \text { (itching and con- } \\
\text { junctival redness) }\end{array}$ & $\begin{array}{l}\mathrm{n}=139 \\
\text { Main variable: Time } \\
\text { course of sum score of } \\
\text { main eye symptoms at } \\
\text { day } 7 \\
\text { Participants' } \\
\text { mean score (no SD) es- } \\
\text { timated from graphs } \\
\text { Secondary outcomes: } \\
\text { symptoms day } 21,42\end{array}$ \\
\hline Davies 1993 & $\begin{array}{l}\text { Area under } \\
\text { the curve for partici- } \\
\text { pant-reported symp- } \\
\text { toms (VAS score 0- } \\
100,0=\text { no symp- } \\
\text { toms, } 100=\text { ex- } \\
\text { tremely severe symp- } \\
\text { toms); Investigator's } \\
\text { symptoms score (oc- } \\
\text { ular irritation, c. red- } \\
\text { ness, itching, photo- } \\
\text { phobia, }\end{array}$ & 14 days & $\begin{array}{l}\text { Participants: VAS } \leq \\
10 \text { symptom-free } 37 \% \\
(P<0.01) \\
\text { VAS } \leq 50 \text { symptom- } \\
\text { free } 40 \% \\
(P<0.1)\end{array}$ & $\begin{array}{l}\text { Participants: VAS } \leq 10 \\
\text { symptom-free } 4 \% \\
(P<0.01) \\
\text { VAS } \leq 50 \text { symptom- } \\
\text { free } 13 \%\end{array}$ & $\begin{array}{l}\mathrm{n}=95 \\
\text { VAS percentages (esti- } \\
\text { mated from graph) } \\
\text { Investigator's } \\
\text { symptoms severity } 0-3 \\
\text { scale }(0=\text { absent, } 3= \\
\text { severe) }\end{array}$ \\
\hline
\end{tabular}

Topical antihistamines and mast cell stabilisers for treating seasonal and perennial allergic conjunctivitis (Review) 


\begin{tabular}{|c|c|c|c|c|c|}
\hline & $\begin{array}{l}\text { ing, lid and conjunc- } \\
\text { tival oedema) (range } \\
0-3 \text { ) }\end{array}$ & & & & \\
\hline Graue 1994 & $\begin{array}{l}\text { Investigator- } \\
\text { reported symptoms } \\
\text { (absent, mild, mod- } \\
\text { erate, or severe scale) } \\
\text { Partici- } \\
\text { pant-reported symp- } \\
\text { toms (VAS scale, } 0 \\
=\text { no symptoms and } \\
10=\text { worst possible } \\
\text { symptoms) }\end{array}$ & 7 days & $\begin{array}{l}\text { Symptoms } \\
\text { improvement: } \\
58 \% \text { first } 2 \text { hours } \\
88 \% \text { day } 1 \\
\text { Percentage at day } 7 \\
\text { to be estimated from } \\
\text { graphs }\end{array}$ & $\begin{array}{l}\text { Symptoms } \\
\text { improvement: } \\
33.5 \% \text { first } 2 \text { hours } \\
61 \% \text { day } 1 \\
\text { Percentage at day } 7 \\
\text { to be estimated from } \\
\text { graphs }\end{array}$ & $\begin{array}{l}\mathrm{n}=40 \\
\text { Unclear whether graph } \\
\text { is participant or inves- } \\
\text { tigator assessment } \\
\text { Percentages of im- } \\
\text { proved symptoms re- } \\
\text { ported (no means and } \\
\text { SD) }\end{array}$ \\
\hline Sabbah 1998 & $\begin{array}{l}\text { Investiga- } \\
\text { tor's responders rate } \\
\text { based on decrease of } \\
\text { at least } 3 \text { points in the } \\
\text { sum symptom score } \\
\text { (itching, conjuncti- } \\
\text { val redness, lacrima- } \\
\text { tion) (range } 0-9 \text { ) be- } \\
\text { tween day } 0 \text { to } 3 \text {; par- } \\
\text { ticipant's composite } \\
\text { sum symptom score } \\
\text { (itching, conjuncti- } \\
\text { val redness, lacrima- } \\
\text { tion) (range } 0-9 \text { ) }\end{array}$ & 3 and 14 days & $\begin{array}{l}\text { Investigator: } \\
\text { Mean sum scores } 1.25 \\
\text { (no SD) at day } 14 \\
\text { Investigator: } \\
\text { Difference in the mean } \\
\text { sum } \\
\text { score (itching, con- } \\
\text { junctival redness, and } \\
\text { lacrimation) } \\
-5.4(2.3) \text { at day } 14\end{array}$ & $\begin{array}{l}\text { Investigator: } \\
\text { Mean sum scores } 2.45 \\
\text { (no SD) at day } 14 \\
\text { Investigator: } \\
\text { Difference in the mean } \\
\text { sum } \\
\text { score (itching, con- } \\
\text { junctival redness, and } \\
\text { tearing) -3.4 (3.0) at } \\
\text { day } 14\end{array}$ & $\begin{array}{l}\mathrm{n}=113 \\
\text { Investigators' mean es- } \\
\text { timated from graph } \\
\text { (no SD) } \\
\text { Participant-reported } \\
\text { data as response rate } \\
\text { ( } \mathrm{n}, \% \text { ) for composite } \\
\text { sum symptom score } \\
\text { (itching, conjunctival } \\
\text { redness, and tearing) at } \\
\text { days } 3 \text { and } 14\end{array}$ \\
\hline
\end{tabular}

Footnotes

SD: standard deviation

VAS: visual analogue scale

\section{Olopatadine versus ketotifen}

The search identified four eligible studies comparing the antihistamines olopatadine and ketotifen (Avunduk 2005; HöfflingLima 2001; Sarker 2011; Varguez-Rodriguez 2009). One study was a three-arm trial comparing both olopatadine and ketotifen with placebo (Avunduk 2005). Drug concentration was the same in all studies (olopatadine $0.1 \%$ and ketotifen $0.025 \%$ ), except Höffling-Lima 2001, which used ketotifen $0.05 \%$.

Duration of treatment was four weeks in three studies, Avunduk 2005, Höflling-Lima 2001, and Varguez-Rodriguez 2009, and two weeks in one study (Sarker 2011).

In all studies the sample size was relatively small. The number of participants randomised to olopatadine and ketotifen were 16 versus 16 (Avunduk 2005), 20 versus 20 (Höffling-Lima 2001), 46 versus 46 (Sarker 2011), and 20 versus 20 (Varguez-Rodriguez 2009), respectively.

\section{Primary outcomes}

Although four studies reported at least two of the four symptoms prespecified as primary outcomes (itching and tearing), there was some variation in how these outcomes were reported.

All four studies collected data on participant-reported itching using a 0-3 scale. Two studies reported mean and SD values (Avunduk 2005; Sarker 2011).

Two studies did not find any differences between olopatadine and ketotifen in itching reporting (Avunduk 2005; Höffling-Lima 2001), while two studies found a greater reduction in itching with olopatadine than with ketotifen after two weeks of treatment (Sarker 2011; Varguez-Rodriguez 2009). Sarker 2011 reported 
two-week mean scores of 1.09 (SD 0.53) with ketotifen and 0.33 (SD 0.60) with olopatadine. A random-effects meta-analysis of these four studies showed evidence of a statistically significant difference in favour of olopatadine in the reduction of itching at 14 days (mean difference (MD) $-0.32,95 \%$ confidence interval (CI) -0.59 to -0.06 ) (Figure 5). However, there was high statistical heterogeneity $\left(I^{2}=83 \%\right)$.

Figure 5. Forest plot of comparison: I Olopatadine versus ketotifen, outcome: I.I Itching at I4 days (0-3 scale)

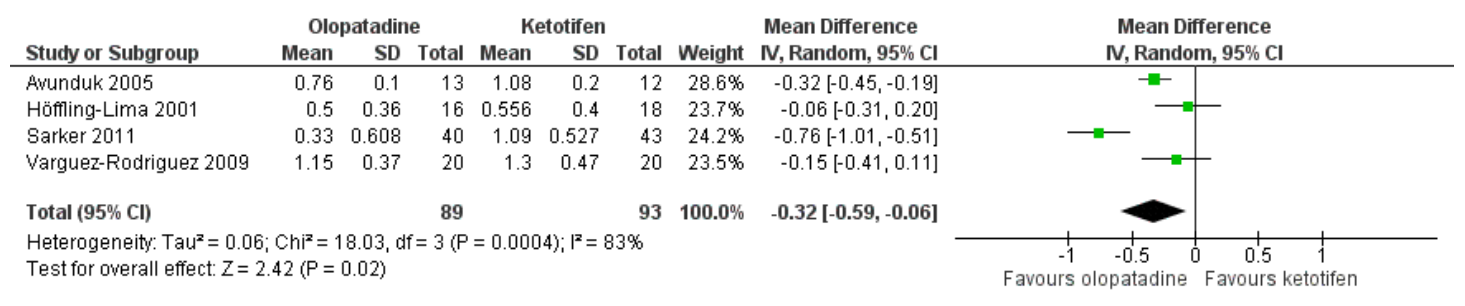

Two studies presented data for participant-reported ocular irritation (described in both studies as burning) (Höffling-Lima 2001; Varguez-Rodriguez 2009). Höffling-Lima 2001 did not find any differences between groups, while Varguez-Rodriguez 2009 reported a statistically significant greater reduction in burning in the olopatadine group $(\mathrm{P}<0.05)$.

All four studies reported data on tearing. Only one study reported differences between groups after two weeks (Sarker 2011), with the group treated with olopatadine having less tearing (mean 0.03, SD
0.16) than the one treated with ketotifen (mean 0.40 , SD 0.66). Three studies assessed tearing at 14 days (Avunduk 2005; HöfflingLima 2001; Sarker 2011). A random-effects meta-analysis of these studies found no evidence of a difference between olopatadine and ketotifen (MD - $0.06,95 \% \mathrm{CI}-0.35$ to 0.22 ). There was no evidence of a difference in the reduction of tearing scores at 14 days between the 2 groups (Figure 6). Once again, there was high statistical heterogeneity between the studies $\left(\mathrm{I}^{2}=90 \%\right)$.

Figure 6. Forest plot of comparison: I Olopatadine versus ketotifen, outcome: I.2 Tearing at I4 days (0-3 scale)

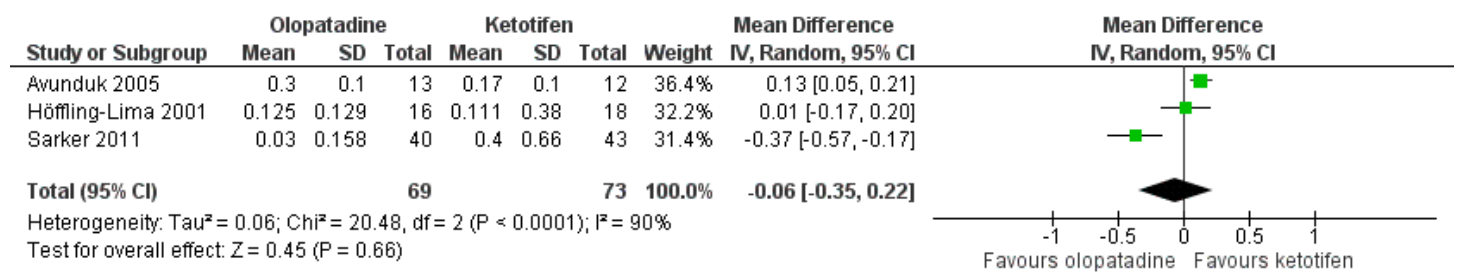

We knew of two studies that collected data on photophobia (Höffling-Lima 2001; Sarker 2011). There were no differences between groups.

\section{Safety outcomes}

No serious adverse events were reported in the four papers. Three studies did not report any side effects (Avunduk 2005; HöfflingLima 2001; Varguez-Rodriguez 2009). In one study, ketotifen was associated with a mild stinging sensation of short duration (less than 30 minutes) in 13 out of 43 participants; no participants

Topical antihistamines and mast cell stabilisers for treating seasonal and perennial allergic conjunctivitis (Review) 
treated with olopatadine reported such discomfort (Sarker 2011).

\section{Overall summary}

There was some evidence from individual trials that olopatadine may be more effective than ketotifen in improving some ocular symptoms such as itching.

Both drugs are safe.

Table 4. Olopatadine versus ketotifen. Outcome definition, time points, and summary of results

\begin{tabular}{|c|c|c|c|c|c|}
\hline Article & $\begin{array}{l}\text { Outcome } \\
\text { definition }\end{array}$ & Time point & Olopatadine & Ketotifen & $\begin{array}{l}\text { Number of partici- } \\
\text { pants randomised (n) } \\
\text { and comment }\end{array}$ \\
\hline Avunduk 2005 & $\begin{array}{l}\text { Participant-re- } \\
\text { ported (itching and } \\
\text { tearing; range 0-3) } \\
\text { and ocular } \\
\text { signs (redness, eye- } \\
\text { lid swelling, chemo- } \\
\text { sis; range } 0-3 \text { ) }\end{array}$ & 15 days & $\begin{array}{l}\text { Itching: } \\
\text { Mean (SD) } 0.76(0.1) \\
\text { Tearing: } \\
\text { Mean (SD) } 0.30(0.1)\end{array}$ & $\begin{array}{l}\text { Itching: } \\
\text { Mean (SD) } 1.08(0.2) \\
\text { Tearing: } \\
\text { Mean (SD) } 0.17(0.1)\end{array}$ & $\begin{array}{l}\mathrm{n}=39 \\
\mathrm{SD} \text { estimated from } \\
\text { graph }\end{array}$ \\
\hline $\begin{array}{l}\text { Höffling-Lima } \\
2001\end{array}$ & $\begin{array}{l}\text { Partici- } \\
\text { pant-reported (itch- } \\
\text { ing, burning, water- } \\
\text { ing, discharge, pho- } \\
\text { tophobia; range 0- } \\
3 \text { ) } \\
\text { Signs, inves- } \\
\text { tigator examination } \\
\text { (range } 0-3)\end{array}$ & 14 days & $\begin{array}{l}\text { Itching: Mean } 0.50 \\
\text { Watering: Mean } 0.15 \\
\text { Burning: Mean } 0.18 \\
\text { Photophobia: Mean } 0 . \\
00\end{array}$ & $\begin{array}{l}\text { Itching: Mean } 0.55 \\
\text { Watering: Mean } 0.11 \\
\text { Burning: Mean } 0.11 \\
\text { Photophobia: Mean } 0 . \\
05\end{array}$ & $\mathrm{n}=40$ \\
\hline Sarker 2011 & $\begin{array}{l}\text { Partici- } \\
\text { pant-reported (itch- } \\
\text { ing, watering, pho- } \\
\text { tophobia; range 0- } \\
\text { 3) } \\
\text { (redness; range } 0-3 \text { ) }\end{array}$ & 14 days & $\begin{array}{l}\text { Itching: Mean (SD): } 0 . \\
33(0.60) \\
\text { Watering: Mean (SD): } \\
0.03(0.15) \\
\text { Photophobia: Mean } \\
(\mathrm{SD}): 0.05(0.22)\end{array}$ & $\begin{array}{l}\text { Itching: Mean (SD): } 1 . \\
09(0.52) \\
\text { Watering: Mean (SD): } \\
0.40(0.66) \\
\text { Photophobia: Mean } \\
\text { (SD): } 0.26(0.44)\end{array}$ & $\mathrm{n}=92$ \\
\hline $\begin{array}{l}\text { Varguez-Rodriguez } \\
2009\end{array}$ & $\begin{array}{l}\text { Partici- } \\
\text { pant-reported (itch- } \\
\text { ing, watering, burn- } \\
\text { ing; range 0-3) } \\
\text { Signs, inves- } \\
\text { tigator examination } \\
\text { (redness, chemosis; } \\
\text { range: present or ab- }\end{array}$ & 14 days & $\begin{array}{l}\text { Data not extractable. } \\
\text { Only P value available. }\end{array}$ & $\begin{array}{l}\text { Data not extractable. } \\
\text { Only P value available. }\end{array}$ & $\begin{array}{l}\mathrm{n}=40 \\
\text { Olopatadine induced } \\
\text { greater reduction of } \\
\text { burning than ketotifen } \\
\text { at } 2 \text { weeks. There was } \\
\text { no difference in itch- } \\
\text { ing and tearing at } 2 \\
\text { weeks }\end{array}$ \\
\hline
\end{tabular}


Footnotes

SD: standard deviation

\section{Emedastine versus levocabastine}

We identified three randomised studies that compared the antihistamines emedastine and levocabastine (or levocabastine) (Secchi 2000a; Secchi 2000b; Verin 2001). There was substantial overlap in the authorship of these three studies, and they had some similarities in study design, outcome measures, and the time points for clinic visits (days 3, 7, 14, 30, and 42). One study, Secchi 2000a, was done entirely in a paediatric population (range 4 to 16 years); the other two studies, although including mainly adults, recruited participants from a wide range of age groups (range 4 to 76 years). The same treatment dose $(0.05 \%$ twice a day $)$ was used in each study, and treatment lasted for six weeks.

The number of participants randomised to emedastine and levocabastine was 20 versus 22 (Secchi 2000a), 97 versus 105 (Secchi 2000b), and 97 versus 105 (Verin 2001), respectively.

\section{Primary outcomes}

All three studies evaluated itching. Secchi 2000a evaluated itching up to 42 days using a $0-9$ scale. At 14 days, mean scores of 0.7 (emedastine) and 2.5 (levocabastine) were obtained (no SD, estimated from graph). Maximum itching scores from participant diaries were also reported as always lower for emedastine for this study, but no actual data were reported. Secchi 2000b also collected itching data at visits and using participant diaries but did not report any specific data in this article. The authors stated that emedastine was statistically significantly better than levocabastine at 4 of the 5 time points evaluated. Using graphs, Verin 2001 reported mean itching and maximum itching (participant diaries) up to 42 days using a $0-4$ scale. At 14 days, mean scores were 1.5 (emedastine) versus 2.3 (levocabastine). For this study, participant diary scores for maximum itching were 2.0 (emedastine) versus 2.7 (levocabastine).

None of the studies directly evaluated the other primary outcomes of this review (irritation, watering eyes, and photophobia).

\section{Overall symptom score}

Secchi 2000a measured the physician's overall impression score at each visit. At 14 days this was 1.1 (emedastine) versus 1.9 (levocabastine) (no SD, estimated from graph). Secchi $2000 \mathrm{~b}$ measured the physician's overall assessment at the same time points, stating that emedastine was statistically significantly better than levocabastine at all 5 time points, but presented no data.

\section{Conclusion}

There was only limited information on our primary outcomes, as these three papers focused on chemosis and eyelid swelling. The results suggested a benefit in favour of emedastine compared with levocabastine for itching and overall symptom relief, but despite some similarities in study design between the three papers, it was not possible to conduct formal meta-analysis, either because the studies presented no data or because they provided no SDs. It was not possible to investigate irritation, watering eyes, or photophobia as no data were available.

Table 5. Emedastine versus levocabastine. Outcome definition, time points, and summary of results

\begin{tabular}{|c|c|c|c|c|c|}
\hline Article & Outcome definition & Time point & Emedastine & levocabastine & $\begin{array}{l}\text { Number of participants randomised (n) } \\
\text { and comment }\end{array}$ \\
\hline Secchi $2000 a$ & $\begin{array}{l}\text { Mean physician's im- } \\
\text { pression score }(0-4 \\
\text { scale) }\end{array}$ & 14 days & 1.1 & 1.9 & $\begin{array}{l}\mathrm{n}=42 \\
\text { Estimated from graph, no standard deviation }\end{array}$ \\
\hline Secchi 2000b & $\begin{array}{l}\text { Mean physician's im- } \\
\text { pression score }(0-4 \\
\text { scale) }\end{array}$ & 14 days & Not reported & Not reported & $\begin{array}{l}\mathrm{n}=202 \\
\text { "statistically significant" differences }\end{array}$ \\
\hline
\end{tabular}

Topical antihistamines and mast cell stabilisers for treating seasonal and perennial allergic conjunctivitis (Review) 


\section{Nedocromil sodium/sodium cromoglycate versus levocabastine}

We identified two randomised studies comparing the mast cell stabiliser sodium cromoglycate with the antihistamine levocabastine (levocabastine) (Azevedo 1991; Davies 1993). Duration of treatment was four weeks in both studies, and both studies also included a placebo arm. We identified no studies comparing nedocromil sodium versus levocabastine.

The number of participants randomised (or analysed if numbers randomised not known) to sodium cromoglycate and levocabastine were 21 versus 18 in Azevedo 1991 and 32 versus 31 in Davies 1993 , respectively.

\section{Primary outcomes}

Neither study presented data on itching. Davies 1993 collected daily data on itching from participant diaries, but specifically reported no data.

Azevedo 1991 presented a graph showing daily mean irritation using a $0-4$ scale: at 14 days mean itching was around 1.0 for sodium cromoglycate and 0.8 for levocabastine, and there were statistically significant between-group differences in the median area under the curve for the entire 28-day follow-up period in favour of levocabastine. Davies 1993 collected information on ocular irritation but again did not report this information directly. Azevedo 1991 reported that the median area under the curve for tearing was similar in each group. Although the study provided no data, Davies 1993 reported that tearing was statistically significantly lower for levocabastine.

For photophobia, Azevedo 1991 reported similar change scores from baseline for the two groups. Davies 1993 did not report any information on this outcome.

\section{Overall symptom score}

Davies 1993 collected the participant and investigator global evaluation of treatment efficacy using a 4-point scale (Table 6). Azevedo 1991 reported the investigator assessment using a similar scale. In each study there was a similar statistically significant difference in the proportion rated as excellent or good by the investigator in favour of levocabastine: Azevedo 1991 (nedocromil sodium: 67\%, levocabastine: 89\%); Davies 1993 (nedocromil sodium: 68\%, levocabastine: 91\%) (Table 6).

Davies 1993 also reported that participants treated with levocabastine were statistically significantly more likely to be "virtually symptom-free".

\section{Overall summary}

We identified only two randomised studies for this comparison. Although these studies collected data for most of the primary outcomes of this review, the data were either not reported or not given in a format suitable for meta-analysis. Both studies reported a difference of over $20 \%$ in favour of levocabastine in those rating the overall treatment efficacy as good or excellent. Although some statistically significant differences were reported suggesting increased efficacy of levocabastine compared with nedocromil sodium, this finding needs to be treated with caution.

Table 6. Nedocromil sodium/sodium cromoglycate versus levocabastine. Outcome definition, time points, and summary of results

\begin{tabular}{|c|c|c|c|c|c|}
\hline Article & Outcome definition & Time point & $\begin{array}{l}\text { Nedocromil sodium / } \\
\text { sodium cromoglycate }\end{array}$ & Levocabastine & $\begin{array}{l}\text { Number of partic- } \\
\text { ipants randomised (n) } \\
\text { and comment }\end{array}$ \\
\hline Azevedo 1991 & $\begin{array}{l}\text { Investigator's global } \\
\text { assessment }\end{array}$ & 28 days & $\begin{array}{l}\text { Excellent/good } \\
\text { response: } 67 \%(14 / 21)\end{array}$ & $\begin{array}{l}\text { Excellent/good } \\
\text { response: } 89 \%(16 / 18)\end{array}$ & $\begin{array}{l}\mathrm{n}=63 \\
\text { Response at last avail- } \\
\text { able assessment. Per- } \\
\text { centage of symptom- } \\
\text { free days also reported }\end{array}$ \\
\hline Davies 1993 & $\begin{array}{l}\text { Par- } \\
\text { ticipants' and investi- } \\
\text { gator's global evalua- } \\
\text { tion of treatment ef- } \\
\text { ficacy (4-point scale) }\end{array}$ & 28 days & $\begin{array}{l}\text { Participant eval- } \\
\text { uation: Excellent/good: } \\
68 \% \text {; Investigator eval- } \\
\text { uation: Excellent/good: } \\
68 \%\end{array}$ & $\begin{array}{l}\text { Participant eval- } \\
\text { uation: Excellent/good: } \\
\text { 87\%; Investigator eval- } \\
\text { uation: Excellent/good: } \\
91 \%\end{array}$ & $\begin{array}{l}\mathrm{n}=95 \\
\text { Individual categories } \\
\text { (participant evaluation) } \\
\text { can be estimated from } \\
\text { graph, and mean score } \\
\text { could be calculated if } \\
\text { considered appropriate }\end{array}$ \\
\hline
\end{tabular}

Topical antihistamines and mast cell stabilisers for treating seasonal and perennial allergic conjunctivitis (Review) 


\section{Azelastine versus levocabastine}

We identified two randomised studies comparing the antihistamines azelastine and levocabastine (Canonica 2003; Sabbah 1998). Both studies also included a placebo group. The duration of treatment was six weeks in Canonica 2003 and two weeks in Sabbah 1998.

The number of participants randomised (or analysed if numbers randomised not known) to azelastine and levocabastine were 57 versus 26 in Canonica 2003 and 51 versus 32 in Sabbah 1998, respectively.

\section{Primary outcomes}

In both studies itching was assessed by both investigators and participants (using diaries), but no results were directly reported. Canonica 2003 reported only a composite score of itching and redness. Sabbah 1998 only reported itching as part of two composite scores comprising three and eight eye symptoms.

Sabbah 1998 only reported the other primary outcomes as part of composite symptom scores. Tearing (tearing) was reported in both composite scores. Foreign body sensation and photophobia were included as part of the eight-item score.

\section{Overall symptom score}

The composite symptom scores reported by the two studies were different. Canonica 2003 used the sum of itching and redness (range 0-6). At 14 days the mean scores from the participant diaries (estimated from a graph) were around 1.6 (azelastine) versus 1.4 (levocabastine). The investigator-reported composite scores at 7 and 21 days were also similar in each group. The participantreported scores were only reported in terms of the proportion of participants improving.

Sabbah 1998 used two composite scores: the first comprised three symptoms (itching, conjunctival redness, and tearing; range 0-9); the second added five additional symptoms (swollen eyelids, foreign body sensation, photophobia, soreness, and discharge/eyelids sticking together). At day 13 scores for the 3-item score (estimated from a graph) were around 1.8 (azelastine) versus 1.5 (levocabastine). The study presented additional results for the numbers responding by day three of treatment.

\section{Overall summary}

Overall, there was no clear evidence of differences between azelastine and levocabastine in either study (Table 7).

Table 7. Azelastine versus levocabastine. Outcome definition, time points, and summary of results

\begin{tabular}{l|l|l|l|l|l}
\hline Article & Outcome definition & Time point & Azelastine & $\begin{array}{l}\text { Levocabastine } \\
\text { and comment }\end{array}$ & $\begin{array}{l}\text { Number of participants randomised (n) } \\
\text { and }\end{array}$ \\
\hline Canonica 2003 & $\begin{array}{l}\text { Itching and redness } \\
\text { (range 0-6) }\end{array}$ & 14 days & 1.6 & 1.4 & $\begin{array}{l}\mathrm{n}=139 \\
\text { Estimated from a graph (no SD) }\end{array}$ \\
\hline Sabbah 1998 & $\begin{array}{l}\text { Itching, conjunctival } \\
\text { redness, and tearing } \\
\text { (range 0-9) }\end{array}$ & 13 days & 1.8 & 1.5 & $\begin{array}{l}\mathrm{n}=113 \\
\text { Estimated from a graph (no SD). A further } \\
\text { composite score comprising eight symptoms } \\
\text { was also presented }\end{array}$ \\
\hline
\end{tabular}

\section{Footnotes}

SD: standard deviation

\section{Olopatadine versus placebo or other control}

The search identified two eligible studies comparing the antihistamine olopatadine versus placebo, in Avunduk 2005, or other control, in Lanier 2001.

The Avunduk 2005 study was a three-arm trial comparing both olopatadine and ketotifen with placebo (Avunduk 2005). Duration of treatment was four weeks, but two-week data were available. The sample size was relatively small, with 16 participants randomised to olopatadine and 17 participants randomised to ar- tificial tears (placebo). Mean and SD data were available.

Lanier 2001 compared the effect of topical olopatadine in people undergoing treatment with an oral antihistamine (loratadine). Duration of treatment was one week. A total of 94 participants ( 49 versus 45 ) were randomised but not masked. No SD data were available.

\section{Primary outcomes}

Avunduk 2005 reported two of the four symptoms prespecified as primary outcomes (participant-reported itching and watering eyes). Data on mean and SD were available. Olopatadine had 
statistically significantly less severe itching (mean 0.76 , SD 0.1 ) and tearing (mean 0.30, SD 0.1) than the placebo group (mean itching 1.85, SD 0.3; mean tearing 1.07, SD 0.2) after two weeks of treatment.

Lanier 2001 included participant-reported itching on a 4-point scale (from 1 to 4 ). After one week, reported itching was less in the group treated with olopatadine (mean 2.21 versus $2.74, \mathrm{P}=$ $0.044)$.

Safety outcomes
There were no adverse events or side effects associated with olopatadine in either study.

\section{Overall summary}

There was evidence from two small trials that olopatadine may be effective in improving some ocular symptoms.

Table 8. Olopatadine versus control. Outcome definition, time points, and summary of results

\begin{tabular}{|c|c|c|c|c|c|}
\hline Article & Outcome definition & Time point & Olopatadine drops & Placebo & $\begin{array}{l}\text { Number of partici- } \\
\text { pants randomised (n) } \\
\text { and comment }\end{array}$ \\
\hline Avunduk 2005 & $\begin{array}{l}\text { Participant-reported } \\
\text { (itching and tearing; } \\
\text { range } 0-3 \text { ) and ocular } \\
\text { signs (redness, eye- } \\
\text { lid swelling, chemo- } \\
\text { sis; range } 0-3 \text { ) }\end{array}$ & 30 days & $\begin{array}{l}\text { Itching: } \\
\text { Mean (SD) } 0.76(0.1) \\
\text { Tearing: } \\
\text { Mean (SD) } 0.30(0.1)\end{array}$ & $\begin{array}{l}\text { Itching: } \\
\text { Mean (SD) } 1.85(0.3) \\
\text { Tearing: } \\
\text { Mean (SD) } 1.07(0.2)\end{array}$ & $\begin{array}{l}\mathrm{n}=39 \\
\mathrm{SD} \text { estimated from } \\
\text { graph }\end{array}$ \\
\hline Lanier 2001 & $\begin{array}{l}\text { Participant-reported } \\
\text { itching (range 1-4) } \\
\text { and clinician exam of } \\
\text { redness }\end{array}$ & 7 days & $\begin{array}{l}\text { Itching: } \\
\text { Mean 2.21 } \\
\text { Redness: } \\
\text { Mean 1.36 }\end{array}$ & $\begin{array}{l}\text { Itching: } \\
\text { Mean } 2.74 \\
\text { Redness: } \\
\text { Mean } 1.55\end{array}$ & $\begin{array}{l}\mathrm{n}=94 \\
\text { No SD available }\end{array}$ \\
\hline
\end{tabular}

Footnotes

SD: standard deviation

\section{Nedocromil sodium or sodium cromoglycate versus azelastine}

We identified a single randomised study comparing the mast cell stabiliser sodium cromoglycate with the antihistamine azelastine (James 2003). This study also included a placebo arm. The original numbers randomised was not clear, but the analysis included 50 (sodium cromoglycate) versus 45 (azelastine) participants. Duration of treatment was two weeks.

\section{Primary outcomes}

Unfortunately this study did not directly report any of our prespecified outcomes at follow-up, although it did report results for itching, foreign body sensation, and photophobia in terms of the improvement rates from baseline to day three. Improvement rates were higher for azelastine for all eight symptoms examined.

\section{Overall symptom score}

James 2003 also reported 2 composite symptom scores comprising 3 main symptoms (itching, redness, and tearing): an investigatorreported score and composite symptom scores from participant diaries up to 14 days. Scores were similar in each group.

The study reported response rates to treatment by day 3 (a decrease of at least 3 points in the composite symptom score) to be 39 out of $47(83 \%)$ for sodium cromoglycate and 35 out of 41 (85\%) for azelastine.

\section{Overall summary}

The evidence for this comparison came from a single study, and although the study reported results for ocular symptoms, the results were not in the format prespecified for this review. Overall, there was no clear evidence of a difference between the two treatments.

\section{Levocabastine versus antazoline plus tetryzoline}


We identified a single randomised study comparing the antihistamine levocabastine $(\mathrm{n}=35)$ versus the antihistamine antazoline plus tetryzoline (a derivative of imidazoline) $(\mathrm{n}=34)$ (Wertheimer 1997).

\section{Primary outcomes}

The study reported no data on the four primary outcomes of this review (itching, irritation, watering eyes, and photophobia). However, all four symptoms were included as part of an overall symptom score.

\section{Overall symptom score}

The study presented a total symptom score (range 0-24) comprising four subjective (itching, foreign body feeling, tearing, and photophobia) and four objective (hyperaemia, follicles, chemosis, and swollen eyelid) symptoms. At day 15 , mean scores were 2.0 for levocabastine and 3.0 for antazoline plus tetryzoline. These results were estimated from a graph, and no SDs were presented. There were no significant differences between the groups at days 4 and 15, although the antazoline/tetryzoline group was favoured when assessed 30 minutes after application.

\section{Overall summary}

Evidence for this comparison comes from one relatively small randomised study. There were no clear differences between the groups.

\section{Ketotifen versus placebo}

The search identified one eligible study comparing the antihistamine ketotifen versus placebo (Avunduk 2005). This study was a three-arm trial comparing both olopatadine and ketotifen with placebo.

Duration of treatment was four weeks.

The sample size was relatively small, with 16 participants randomised to ketotifen and 17 participants randomised to artificial tears (placebo).

\section{Primary outcomes}

The study reported two of the four symptoms prespecified as primary outcomes (itching and watering eyes). Data on mean and SD were available.

Ketotifen had statistically significantly less severe itching (mean 1.08 , SD 0.2) and tearing (mean 0.17, SD 0.1) than the placebo group (mean itching 1.85, SD 0.3; mean tearing 1.07, SD 0.2) after two weeks of treatment.

\section{Safety outcomes}

No adverse events or side effects were reported.

\section{Overall summary}

There is evidence from a small individual trial that ketotifen may be more effective than placebo in improving some ocular symptoms.

\section{Olopatadine versus nedocromil sodium}

The search identified one eligible study comparing the antihistamine olopatadine with the mast cell stabiliser nedocromil sodium in a parallel-group trial where one group used olopatadine and placebo and the other nedocromil sodium (Katelaris 2002).

Duration of treatment was six weeks. The sample size was relatively large, with 91 participants randomised to the olopatadine group and 94 participants randomised to the nedocromil sodium group; analysis was only performed on 82 and 87 participants, respectively. In the olopatadine group, participants were treated with olopatadine $0.1 \%$ (in the morning and evening) and placebo (at noon and afternoon); in the nedocromil sodium group, participants were treated with nedocromil sodium $2 \%$. The study provided no information on how many participants were randomised within each treatment group.

\section{Primary outcomes}

The study reported one of the four symptoms prespecified as primary outcomes (itching). Participants recorded itching and redness on a scale of 0 to 9 ( 9 being more severe). After two weeks, mean itching score of participants taking olopatadine was 1.9, compared to 2.5 in those taking nedocromil sodium $(\mathrm{P}<0.05)$. However, there was no significant statistical difference in redness scores at 14 days.

The study reported that the likelihood of a day without eye redness and itching was 1.6 times greater on most days in participants treated with olopatadine compared to those treated with nedocromil sodium. This difference was recorded as constant over time $(\mathrm{P}<0.001)$.

\section{Safety outcomes}

In the olopatadine group, four cases of treatment-related adverse events were recorded (ocular discharge, stinging, and blurred vision). Two participants experienced dry nose and taste perversion. In the nedocromil sodium group, five participants experienced treatment-related ocular adverse events (dry eye, stinging, itching, and tearing). 


\section{Overall summary}

This study suggested there is some evidence supporting olopatadine as a more effective agent than nedocromil sodium in improving some ocular symptoms of allergic conjunctivitis.

\section{Ketotifen versus levocabastine}

The search identified one eligible study comparing the antihistamine ketotifen $(0.025 \%)$ with the antihistamine levocabastine $(0.05 \%)$ (Kidd 2003). The study was a three-arm trial comparing both ketotifen and levocabastine to placebo in a large, multicentre trial.

Duration of treatment was four weeks.

The sample size was large, with a total of 519 participants being randomised to 1 of 3 groups: 172 participants were randomised to ketotifen and 174 participants were randomised to levocabastine.

\section{Primary outcome}

This study reported only two of the prespecified primary outcome measures (participant-reported itching and watering). Participantrecorded data was only available for days one to four. Participants scored itching on a 5-point scale ( 4 being most severe) and watering on a 4-point scale (3 being severe).

At day 4, mean score of itching for ketotifen was the lowest (1.4), compared with 1.7 for both levocabastine and placebo $(\mathrm{P}<0.05)$. The mean score of watery eyes for participants treated with ketotifen was the lowest (0.75) after 4 days compared with 1.2 for participants treated with both placebo and levocabastine $(\mathrm{P}<0.05)$.

\section{Safety outcomes}

The study recorded four serious adverse events. In the placebo group, two participants experienced persistent photophobia and conjunctivitis with corneal ulcer. In the ketotifen group, two participants experienced spontaneous pneumothorax and abdominal pain, which was probably not due to the drug.

\section{Overall summary}

This study showed some evidence that ketotifen may be superior in alleviating some ocular symptoms of allergic conjunctivitis.

\section{Combined levocabastine hydrochloride and pemirolast potassium versus levocabastine hydrochloride alone}

The search identified one eligible study comparing a combination of the antihistamine levocabastine hydrochloride $(0.025 \%)$ ophthalmic suspension and pemirolast potassium solution with levocabastine $(0.025 \%)$ alone (Fujishima 2008).

Duration of treatment was one week.
The sample size was small, with 15 participants randomised to the combined treatment group and 17 participants randomised to single-agent treatment.

\section{Primary outcomes}

The study reported two of the four symptoms prespecified as primary outcomes (itching, tearing). The study collected data by participant diary and visual analogue scale quantified by the participant. Data on mean and SD was available.

After one week of treatment there was a reduction in mean (SD) itching in both combined-treatment $(-4.6(2.3))$ and single-treatment $(-2.8$ (2.8)) groups. There was no significant difference in alleviation of symptoms between the groups ( $\mathrm{P}=0.079)$.

Only 11 participants in the single-treatment group and 8 participants in the combined-treatment group had symptoms of tearing. The degree of reduction in tearing (mean (SD)) was more statistically significantly in the combined-treatment group compared with the single-treatment group (-4.0 (2.6) vs. -1.5 (0.9), P $=0.008$ ).

\section{Safety outcomes}

No adverse reactions were observed in either group.

\section{Overall summary}

There was some evidence from a single study with a small sample size that levocabastine in combination with pemirolast potassium may be more effective in eliminating some symptoms when compared with levocabastine alone.

\section{Levocabastine versus mequitazine}

The search identified one randomised study as an abstract (full text was not available) comparing the antihistamine levocabastine $(0.05 \%)$ versus the antihistamine mequitazine $(0.05 \%)$ eyedrops in a parallel-group trial (Trinquand 1999).

The study did not report the numbers randomised to each group, but reported that a total of 357 participants were randomised. Duration of treatment was four weeks (doses were twice a day for the first week and two or three times daily for three weeks).

\section{Primary outcomes}

Ocular symptoms were assessed by the investigator and participants (diary cards). Investigator assessment was good or excellent in $72 \%$ of mequitazine-treated participants and $70 \%$ of levocabastine-treated participants. 


\section{Safety outcomes}

The study reported fewer adverse events in the mequitazine group $(\mathrm{P}<0.05)$.

The overall safety of mequitazine $(0.05 \%)$ was significantly better than levocabastine.

\section{Overall summary}

There was some evidence from a single study with a large sample size $(\mathrm{n}=357)$ that mequitazine is as effective as levocabastine in teenagers and adult patients.

\section{Bepotastine besilate versus olopatadine}

The search identified one eligible study comparing the antihistamine bepotastine besilate ophthalmic solution (BBOS) against the antihistamine olopatadine, an investigator-masked, single-centre, cross-over study that randomised 30 participants (McCabe 2012). Participants were not masked.

The duration of treatment was two weeks, followed by a sevenday washout period. After the washout period, participants were crossed-over to the alternative treatment for two additional weeks. Although a paired t-test was used to analyse this study, no paired data were presented. Some results were obtainable from graphs, but these were assumed to represent mean (SD) values after pooling data from both the first and second periods of the study. This meant that unit of analysis issues would have been a potential concern when combining with participant-randomised studies. However, no meta-analyses were possible for this comparison.

\section{Primary outcomes}

Participants assessed ocular itching during the three clinic visits using a 5 -point Likert scale ( $1=$ lowest relief, $5=$ highest relief) and used a daily home diary to assess ocular itch relief twice a day (morning and evening) over two weeks of treatment.

Rather different mean (SD) results were obtained for the morning and evening (morning: BBOS 4.04 (0.12) versus olopatadine 4.10 (0.15); evening: BBOS 4.04 (0.12) versus olopatadine 3.90 $(0.12)$ ). These results appeared to be for both periods of the crossover study combined.

\section{Safety outcomes}

The study reported no serious adverse events. About $10 \%$ of the participants treated with BBOS $1.5 \%$ reported a mild, temporary adverse taste after instillation.

\section{Overall symptom score}

The study reported the mean (SD) rating of each treatment's ability to relieve all ocular-related allergy symptoms ( 1 = lowest relief,
3 = highest relief) for morning and evening separately (morning: BBOS 2.30 (0.1) versus olopatadine 2.25 (0.15); evening: BBOS $2.30(0.1)$ versus olopatadine $2.15(0.15))$. From the participants' diary responses, BBOS $1.5 \%$ was significantly more effective at relieving morning and evening ocular allergy symptoms $(\mathrm{P}=0.032$ and $\mathrm{P}<0.0001$, respectively) compared to olopatadine hydrochloride $0.2 \%$.

\section{Overall summary}

There was insufficient evidence to compare the efficacy of BBOS against olopatadine, as we found only one study with a small sample size that was judged to be at high risk of bias, as participants were not masked to treatment allocation.

\section{BBOS versus placebo}

The search identified a single multicentre, randomised trial comparing the antihistamine BBOS against placebo (Carr 2013). The study randomised participants to receive either BBOS $(n=123)$ or placebo $(n=122)$ twice a day for a period of two weeks.

\section{Primary outcomes}

Participants assessed ocular itching twice a day using a 4-point scale $(0=$ absent, 3 = severe $)$ that was part of the participant outcomes assessed from a Rhinoconjunctivitis Quality of Life Questionnaire. The study reported results as percentages of improvement from baseline (instantaneous -- approximately 15 minutes before scoring, and reflective -- last dosing throughout the 2-week treatment period) of ocular itching scores over the treatment period. The mean change from baseline involved taking the daily average of a two-week period and comparing with the mean scores from a three-day baseline period. For reflective itching, mean improvements were $28.0 \%$ for BBOS and $21.1 \%$ for placebo. For instantaneous itching, mean improvements were $28.3 \%$ for BBOS and $20.3 \%$ for placebo.

\section{Safety outcomes}

The study reported no serious adverse events. More mild adverse events were reported in the BBOS group (29 participants) than in the placebo group (11 participants). The most frequently reported adverse events in the BBOS group were bitter taste (14 participants in the BBOS group, 1 participant in the placebo group) and instillation site pain.

\section{Overall summary}

There was some evidence from a single trial with a large sample size $(\mathrm{n}=245)$ that BBOS is more effective at improving ocular itching than placebo. However, full results other than percentage change in itching scores were not available. 


\section{ISCUSSION}

\section{Summary of main results}

We identified 30 trials with 17 different treatment comparisons evaluating the efficacy and safety of topical antihistamines and mast cell stabilisers, either alone or in combination. The following antihistamines and mast cell stabilisers were evaluated in at least one randomised controlled trial: nedocromil sodium or sodium cromoglycate, olopatadine, ketotifen, azelastine, emedastine, levocabastine or levocabastine, combination of antazoline and tetryzoline, combination of levocabastine and pemirolast potassium, and bepotastine besilate. The most common comparison was azelastine versus placebo (nine studies). Unfortunately, formal metaanalysis was only possible for two outcomes in one comparison (olopatadine versus ketotifen, itching and tearing at 14 days), and the results should be interpreted with caution due to the high statistical heterogeneity, both for itching $\left(\mathrm{I}^{2}=83 \%\right)$ and tearing $\left(\mathrm{I}^{2}\right.$ $=90 \%)$. For this comparison, one study differed from the other studies as it favoured olopatadine (Sarker 2011).

The inability to meta-analyse other results was mainly due to the variety of outcome measures reported and the fact that standard deviations were often not provided. Due to the presence of a network of different treatments in this review, this should have been an ideal situation to conduct a network meta-analysis of overall symptom scores, but the heterogeneity in outcome definitions and time points and the lack of standard deviations meant that sadly, this was not feasible. We did not do sensitivity analysis because of the small number of studies involved in the meta-analysis.

There was some evidence to support the ability of topical antihistamines to reduce symptoms and signs of seasonal allergic conjunctivitis when compared with placebo. There were no serious adverse events related to the use of topical antihistamine treatment.

When comparing different types of antihistamines and mast cell stabilisers, there were limited data to inform if some treatments are more effective than others. Results from a meta-analysis suggested that olopatadine may be more effective than ketotifen in relieving itching, although there was high statistical heterogeneity between the two studies. Results from individual studies suggested better outcomes with emedastine, sodium cromoglycate, and ketotifen when compared with levocabastine. A single study suggested a benefit of olopatadine over sodium cromoglycate.

\section{Overall completeness and applicability of evidence}

There are no long-term studies on the efficacy of topical antihistamines for the treatment of seasonal allergic conjunctivitis, and little evidence to compare antihistamines.

\section{Quality of the evidence}

Poor quality of reporting challenged the synthesis of evidence. We observed a large variability in reporting outcomes. The overall quality of the studies and reporting was poor, and most studies had small sample sizes. Trials only evaluated short-term effects, with a range of treatment of one to eight weeks.

\section{Potential biases in the review process}

None. This review was performed to Cochrane standards.

\section{Agreements and disagreements with other studies or reviews}

We are not aware of any other similar reviews.

\section{A U THORS, CONCLUSIONS}

\section{Implications for practice}

Topical antihistamines and mast cell stabilisers reduce symptoms and signs of seasonal allergic conjunctivitis when compared with placebo in the short term. Overall, topical antihistamines and mast cell stabilisers appear to be safe and well tolerated. There is poor evidence to compare efficacy among different antihistamines and mast cell stabilisers.

\section{Implications for research}

Methodological research to reach consensus on core outcome measures and how best to quantify them would facilitate research in this area. Large trials comparing the efficacy of different antihistamines and mast cell stabilisers would be required.

\section{ACKNOW LEDGEMENTS}

The Cochrane Eyes and Vision editorial team prepared and executed the electronic searches for the review and provided support during the preparation of the protocol and the review. We thank Fiona Stewart, Information Specialist at the Health Services Research Unit, for devising and running a manual search strategy for finding systematic reviews. 


\section{R E F E R E N C E S}

\section{References to studies included in this review}

Avunduk 2005 \{published data only\}

* Avunduk AM, Tekelioglu Y, Turk A, Akyol N.

Comparison of the effects of ketotifen fumarate $0.025 \%$ and olopatadine $\mathrm{HCl} 0.1 \%$ ophthalmic solutions in seasonal allergic conjunctivities: a 30-day, randomized, doublemasked, artificial tear substitute-controlled trial. Clinical Therapeutics 2005;27(9):1392-402.

Azevedo 1991 \{published data only\}

* Azevedo M, Castel-Branco MG, Ferraz Oliveira J, Ramos E, Delgado L, Almeida J. Double-blind comparison of levocabastine eye drops with sodium cromoglycate and placebo in treatment of seasonal conjunctivitis. Clinical and Experimental Allergy 1991;21(6):689-94.

Canonica 2003 \{published data only\}

* Canonica GW, Ciprandi G, Petzold U, Kolb C, Ellers-

Lenz B, Hermann R. Topical azelastine in perennial allergic conjunctivitis. Current Medical Research \& Opinion 2003; 19(4):321-9.

Carr 2013 \{published data only\}

* Carr WW, Nayak AS, Ratner PH, Gow JA, McNamara TR, Williams JI, et al. Efficacy of Bepotastine besilate ophthalmic solution $1.5 \%$ for seasonal allergic conjunctivitis: A randomized, placebo-controlled, natural exposure, clinical trial. Allergy Asthma Proceedings 2013;34 (3):247-54.

Davies 1993 \{published data only\}

* Davies BH, Mullins J. Topical levocabastine is more effective than sodium cromoglycate for the prophylaxis and treatment of seasonal allergic conjunctivitis. Allergy 1993; 48(7):519-24.

Fujishima 2008 \{published data only\}

* Fujishima H, Fukagawa K, Tanaka M, Uchio E, Takamura E, Nakagawa $Y$, et al. The effect of a combined therapy with a histamine $\mathrm{H} 1$ antagonist and a chemical mediator release inhibitor on allergic conjunctivitis. Ophthalmologica 2008; 222(4):232-9.

Giede-Tuch 1998 \{published data only\}

* Giede-Tuch C, Westhoff M, Zarth A. Azelastine eye-drops in seasonal allergic conjunctivitis or rhinoconjunctivitis. A double-blind, randomized, placebo-controlled study. Allergy 1998;53(9):857-62.

Graue 1994 \{published data only\}

* Graue E, Garcia-Valenzuela E. Double-masked study of topical levocabastine versus topical placebo in management of seasonal conjunctivitis [Estudio doble ciego de levocabastina tópica contra placebo tópico en el manejo de las conjuntivits primaverales]. Investigacion Medica Internacional 1994;21(1):35-42.

Hechanova 1984 \{published data only\}

* Hechanova M. A double-blind study comparing sodium cromoglycate eye ointment with placebo in the treatment of chronic allergic conjunctivitis. Clinical Trials Journal 1984; 21(2):59-66.

Höffling-Lima 2001 \{published data only\}

* Höffling-Lima AL, Andrade AJ, Marback PM, Farah ME, Mascaro V. Comparison between topical use of ketotifen and olopatadine in the treatment of allergic conjunctivitis [Comparação do uso tópico de cetotifeno com a olopatadina no tratamento de conjuntivites alérgicas]. Arquivos Brasileiros de Oftalmologia 2001;64(5):415-22.

James 2003 \{published data only\}

* James IG, Campbell LM, Harrison JM, Fell PJ, Ellers-Lenz B, Petzold U. Comparison of the efficacy and tolerability of topically administered azelastine, sodium cromoglycate and placebo in the treatment of seasonal allergic conjunctivitis and rhinoconjuctivitis. Current Medical Research and Opinion 2003;19(4):313-20.

\section{Katelaris 2002 \{published data only\}}

* Katelaris CH, Ciprandi G, Missotten L, Turner FD, Bertin $D$, Berdeaux $G$, et al. A comparison of the efficacy and tolerability of olopatadine hydrochloride $0.1 \%$ ophthalmic solution and cromolyn sodium $2 \%$ ophthalmic solution in seasonal allergic conjunctivitis. Clinical Therapeutics 2002; 24(10):1561-75.

\section{Kidd 2003 \{published data only\}}

* Kidd M, Mckenzie SH, Steven I, Cooper C, Lanz R, Australian Ketotifen Study Group. Efficacy and safety of ketotifen eye drops in the treatment of seasonal allergic conjunctivitis. British Journal of Ophthalmology 2003;87 (10):1206-11.

Lanier 2001 \{published data only\}

* Lanier BQ, Gross RD, Marks BB, Cockrum PC, Juniper EF. Olopatadine ophthalmic solution adjunctive to loratadine compared with loratadine alone in patients with active seasonal allergic conjunctivitis symptoms. Annals of Allergy, Asthma and Immunology 2001;86(6):641-8.

Leino 1992 \{published data only\}

* Leino M, Ennevaara K, Latvala AL, Nordgren P, Posti AM, Suves R, et al. Double-blind group comparative study of $2 \%$ nedocromil sodium eye drops with $2 \%$ sodium cromoglycate and placebo eye drops in the treatment of seasonal allergic conjunctivitis. Clinical and Experimental Allergy 1992;22(10):929-32.

Lenhard 1997 \{published data only\}

* Lenhard G, Mivsek-Music E, Perrin-Fayolle M, Obtulowicz K, Secchi A. Double-blind, randomised, placebo-controlled study of two concentrations of azelastine eye drops in seasonal allergic conjunctivitis or rhinoconjunctivitis. Current Medical Research and Opinion 1997;14(1):21-8.

McCabe 2012 \{published data only\} ${ }^{*}$ McCabe CF, McCabe SE. Comparative efficacy of bepotastine besilate $1.5 \%$ ophthalmic solution versus olopatadine hydrochloride $0.2 \%$ ophthalmic solution 
evaluated by patient preference. Clinical Ophthalmology 2012;6:1731-8.

Melamed 1994 \{published data only\}

Melamed J, Schwartz RH, Hirsch SR, Cohen SH. Evaluation of nedocromil sodium 2\% ophthalmic solution for the treatment of seasonal allergic conjunctivitis. Annals of Allergy 1994;73(1):57-66.

Melamed 2000 \{published data only\}

* Melamed J, Schwartz RH, Blumenthal MN, Zeitz HJ. Efficacy and safety of nedocromil sodium 2\% ophthalmic solution b.i.d. in the treatment of ragweed seasonal allergic conjunctivitis. Allergy and Asthma Proceedings 2000;21(4): 235-9.

Möller 1994 \{published data only\}

* Möller C, Berg IM, Berg T, Kjellman M, Strömberg L. Nedocromil sodium 2\% eye drops for twice-daily treatment of seasonal allergic conjunctivitis: a Swedish multicentre placebo-controlled study in children allergic to birch pollen. Clinical and Experimental Allergy 1994;24(9):884-7.

Nazarov 2003 \{published data only\}

* Nazarov O, Petzold U, Haase H, Nguyen DT, Ellerz-

Lenz B, Hermann R. Azelastine eye drops in the treatment of perennial allergic conjunctivitis. Arzneimittel-Forschung 2003;53(3): 167-73

Petzold 2002 \{published data only\}

Petzold U, Blochin BM, Zimmerman T, Sabbah A, Karafilidis J, Lavallee N, et al. Efficacy and safety of azelastine in allergic conjunctivitis in children. Journal of Allergy and Clinical Immunology 2002;109(Suppl 1):162.

Sabbah 1998 \{published data only\}

* Sabbah A, Marzetto M. Azelastine eye drops in the treatment of seasonal allergic conjunctivitis or rhinoconjunctivitis in young children. Current Medical Research and Opinion 1998;14(3):161-70.

Sarker 2011 \{published data only\}

* Sarker S, Chowdhury AN, Hussain Z, Mosharrof Hossain AK, Chowdhury H. Comparison of the therapeutic efficacy of $0.1 \%$ olopatadine hydrochloride and $0.025 \%$ ketotifen fumarate in allergic conjunctivitis. Therapy 2011;8(5): $545-53$.

Secchi 2000a \{published data only\}

Secchi A, Ciprandi G, Leonardi A, Deschenes J, Abelson MB, Emadine Study Group. Safety and efficacy comparison of emedastine $0.05 \%$ ophthalmic solution compared to levocabastine $0.05 \%$ ophthalmic suspension in pediatric subjects with allergic conjunctivitis. Acta Ophthalmologica Scandinavica. Supplement 2000;78:42-7.

Secchi 2000b \{published data only\}

Secchi A, Leonardi A, Discepola M, Deschenes J, Abelson MB, Emadine Study Group. An efficacy and tolerance comparison of emedastine difumarate $0.05 \%$ and levocabastine hydrochloride $0.05 \%$ : reducing chemosis and eyelid swelling in subjects with seasonal allergic conjunctivitis. Acta Ophthalmologica Scandinavica. Supplement 2000;78:48-51.
Trinquand 1999 \{published data only\}

Trinquand C, Belayachi N, Hoang-Xuan T, International Mequitazine Investigators Group. Clinical double masked comparison of Mequitazine eyedrops $0.05 \%$ compared to Levocabastine eyedrops $0.05 \%$ in allergic conjunctivitis. Investigative Ophthalmology and Visual Science 1999; Vol. 40:ARVO E-Abstract 4819

Varguez-Rodriguez 2009 \{published data only\}

* Varguez-Rodriguez ME, Hernández-López RA, GómezDávila R. Olopatadine and ketotifen for the treatment of allergic conjunctivitis [Olopatadina y ketotifeno para tratar conjuntivitis alérgica]. Revista Médica del Instituto Mexicano del Seguro Social 2009;47(4):399-404.

Verin 2001 \{published data only\}

* Verin P, Eatsy DL, Secchi A, Ciprandi G, Partouche P, Nemeth-Wasmer G, et al. Clinical evaluation of twice-daily emedastine $0.05 \%$ eye drops (emadine eye drops) versus levocabastine $0.05 \%$ eye drops in patients with allergic conjunctivitis. American Journal of Ophthalmology 2001; 131(6):691-8.

Wertheimer 1997 \{published data only\}

* Wertheimer R, Ble $\beta$ mann G. Antazolin/tetryzolineyedrops in comparison to levocabastine-eyedrops in acute allergic conjunctivitis [Antazolin/tetryzolinhaltige augentropfen im vergleich zu levocabastinhaltigen augentropfen bei akuter allergischer Konjunktivitis]. Klinische Monatsblatter fur Augenheilkunde 1997;210(2): 93-6.

\section{References to studies excluded from this review}

\section{Abelson 2003 \{published data only\}}

Abelson MA, Ghosh P, Bradford R, Kim B, Schiffman R, Whitcup S. An environmental study of ophthalmic epinastine in patients with allergic conjunctivitis. Journal Allergy Clinical Immunology 2003;111:2.

\section{Abelson 2004 \{published data only\}} Abelson MB, Gomes PJ, Vogelson CT, Pasquine TA, Gross $\mathrm{RD}$, Turner FD, et al. Clinical efficacy of olopatadine hydrochloride ophthalmic solution $0.2 \%$ compared with placebo in patients with allergic conjunctivitis or rhinoconjunctivitis: a randomized, double-masked environmental study. Clinical Therapeutics 2004;26(8): 1237-48.

Artal 2000 \{published data only\} Artal MN, Luna JD, Discepola M. A forced choice comfort study of olopatadine hydrochloride $0.1 \%$ versus ketotifen fumarate $0.05 \%$. Acta Ophthalmologica Scandinavica. Supplement 2000;78:64-5.

\section{Borazan 2009 \{published data only\}}

Borazan M, Karalezli A, Akova YA, Akman A, Kiyici H, Erbek S. Efficacy of olopatadine HCI $0.1 \%$, ketotifen fumarate $0.025 \%$, epinastine HCI $0.05 \%$, emedastine $0.05 \%$ and fluorometholone acetate $0.1 \%$ ophthalmic solutions for seasonal allergic conjunctivitis: a placebocontrolled environmental trial. Acta Ophthalmologica 2009; 87(5):549-54. 
Garay 2001 \{published data only\}

Garay RP, Doucet P, Garay ER, Deschamps E, Dureau P, Baehre M, et al. Seasonal allergic conjunctivitis: a pilot study of the clinical efficacy of azelastine eye drops in comparison with nedocromil. Annals of Allergy, Asthma and Immunology 2001;86:114.

Higuchi 1979 \{published data only\}

Higuchi M, Ohno S, Minami H, Matsuda H. Clinical trial of disodium cromoglycate and AA-344 in allergic conjunctivitis. Journal of Clinical Ophthalmology 1979;3 (12):1501-4.

Kamis 2006 \{published data only\}

Kamis U, Ozturk BT, Ozkagnici A, Gunduz K. Comparison of the efficacy of olopatadine hydrochloride $0.1 \%$ ophthalmic solution and artificial tears in seasonal allergic conjunctivitis. Acta Ophthalmologica Scandinavica 2006;84 (1):147-8.

Leino 1994 \{published data only\}

Leino M, Montan P, Njå F. A double-blind group comparative study of ophthalmic sodium cromoglycate, $2 \%$ four times daily and $4 \%$ twice daily, in the treatment of seasonal allergic conjunctivitis. Allergy 1994;49(3):147-51.

Leonardi 2004 \{published data only\}

Leonardi A, Zafirakis P. Efficacy and comfort of olopatadine versus ketotifen ophthalmic solutions: a double-masked, environmental study of patient preference. Current Medical Research \& Opinion 2004;20(8):1167-73.

Longo 1979 \{published data only\}

Longo L, Patruno D, Scaramuzzi O, Foshino Barbaro M. Sodium cromoglycate in allergic conjunctivitis. Treatment experience [Il sodiocromoglicato nella congiuntivite allergica: esperienze di trattamento]. Folia Allergologica et Immunologica Clinica 1979;26(5):425.

Merayo 2003 \{published data only\}

Merayo J, Montero J, Sainz de la Maza MT, Fuster E, Lladonosa A. Effectiveness and impact in the quality of life of Ketotifen ophthalmic solution. Results of Zeta study in patients with seasonal allergic conjunctivitis [Efectividad e impacto en la calidad de vida del colirio de Ketotifeno. Resultados del estudio Zeta en pacientes con conjuntivitis alérgica estacional]. Archivos de la Sociedad Española de Oftalmologia 2003;78(8):433-41.

Möller 1990 \{published data only\}

Möller C, Blychert LO. Levocabastine eyedrops in comparison with cromoglycate in the treatment of conjunctivitis in children with birch pollinosis. Pediatric Allergy and Immunology 1990;1:87-9.

Napoli 2005 \{published data only\} Napoli G, Allegri P, Musso C, Morchio A, Callegarini L, Murialdo U, et al. Long-term follow-up of allergic conjunctivitis in children: the outpatients' department experience (allergic conjunctivitis in children: management and care). European Annals of Allergy and Clinical Immunology 2005;37(1):21-3.
Pinto 2001 \{published data only\}

Pinto CG, Lafuma A, Fagnani F, Nuijten MJC, Berdeaux G. Cost effectiveness of emedastine versus levocabastine in the treatment of allergic conjunctivitis in 7 European countries. Pharmacoeconomics 2001;19(3):255-65.

Scadding 1999 \{published data only\} Scadding GK, Tasman AJ, Murrieta-Aguttes M, Bachert C, The Riperex Study Group. Mizolastine is effective and well tolerated in long-term treatment of perennial allergic rhinoconjunctivitis. Riperex Study Group. Journal of International Medical Research 1999;27(6):273-85.

Scoper 2007 \{published data only\}

Scoper S, Berdy GJ, Lichtenstein SJ, Rubin JM, Bloomenstein M, Prouty RE, et al. Perception and quality of life associated with the use of olopatadine $0.2 \%$ (Pataday) in patients with active allergic conjunctivitis. Advances in Therapy 2007;24(6):1221-32.

Torkildsen 2008 \{published data only\} Torkildsen GL, Ousler III GW, Gomes P. Ocular comfort and drying effects of three topical antihistamine/mast cell stabilizers in adults with allergic conjunctivitis: a randomized, double-masked crossover study. Clinical Therapeutics 2008;30(7):1264-71.

\section{References to studies awaiting assessment}

\section{Dharmistha 2013 \{published data only\}}

Dharmistha P, Sarala N, Datti NP. Comparative study of topical olopatadine hcl $0.1 \%$ with ketotifen fumarate $0.025 \%$ in treatment of allergic conjunctivitis. Indian Journal of Pharmacology Conference: 46th Annual Conference of the Indian Pharmacological Society, IPSCON; 2013 Dec 16-18; Bangalore. 2013.

Jia 2012 \{published data only\} Jia HL, Liu CM, Deng HW, Han B, Zhu XL. Clinical study of Azelastine eye drops in children with allergic conjunctivitis. International Eye Science 2012; Vol. 12, issue 4:780-1.

Scandashree 2013 \{published data only\} Scandashree K, Patil M, Udaykumar P, Mendonca N. Comparison of efficacy and tolerability of olopatadine hydrochloride $0.2 \%$ ophthalmic solution Od and sodium cromoglycate $2 \%$ ophthalmic solution qid in allergic conjunctivitis - A randomized, open label, prospective study. Indian Journal of Pharmacology Conference: 46th Annual Conference of the Indian Pharmacological Society, IPSCON; 2013 Dec 16-18; Bangalore. 2013.

\section{Additional references}

\section{Abelson 1979}

Abelson MB, Allansmith MR. Histamine in the eye. In: Silverstein A, O'Connor G editor(s). Immunology and Immunopathology of the Eye. New York: Masson Publishing, 1979:362-4. 


\section{Anderson 2001}

Anderson DF. Management of seasonal allergic

conjunctivitis (SAC): current therapeutic strategies. Clinical and Experimental Allergy 2001;31(6):823-6.

\section{Beasley 1998}

Beasley, R, The International Study of Asthma and Allergies in Childhood (ISAAC) Steering Committee. Worldwide variation in prevalence of symptoms of asthma, allergic rhinoconjunctivitis, and atopic eczema: ISAAC. Lancet 1998;351(9111):1225-32.

Buckley 1998

Buckley R. Allergic eye disease. In: Durham SR editor(s). ABC of Allergies. London: BMJ Books, 1998:23-5.

\section{Cook 2002}

Cook EB, Stahl JL, Barney NP, Graziano FM. Mechanisms of antihistamines and mast cell stabilizers in ocular allergic inflammation. Current Drug Targets. Inflammation and Allergy 2002;1(2):167-80.

\section{Dart 1986}

Dart JK. Eye disease at a community health centre. British Medical Journal 1986;293(6560):1477-80.

Glanville 2006

Glanville JM, Lefebvre C, Miles JN, Camosso-Stefinovic J. How to identify randomized controlled trials in MEDLINE: ten years on. Journal of the Medical Library Association 2006; 94(2):130-6.

\section{Higgins 2011a}

Higgins JPT, Altman DG, Sterne JAC (editors). Chapter 8: Assessing risk of bias in included studies. In: Higgins JPT, Green S (editors). Cochrane Handbook for Systematic Reviews of Interventions Version 5.1.0 (updated March 2011). The Cochrane Collaboration, 2011. Available from www.cochrane-handbook.org.

Higgins 2011b

Higgins JPT, Deeks JJ, Altman DG (editors). Chapter 16. Special topics in statistics. In: Higgins JPT, Green $S$ (editors). Cochrane Handbook for Systematic Reviews of Interventions Version 5.1.0 (updated March 2011). The Cochrane Collaboration, 2011. Available from www.cochrane-handbook.org.

\section{Hingorani 1997}

Hingorani M, Lightman S. Ocular allergy. In: Kay AB editor(s). Allergy and Allergic Disease. Oxford: Blackwell Science, 1997:1645-70.

\section{Leonardi 2005}

Leonardi A. Emerging drugs in ocular allergy. Expert

Opinion on Emerging Drugs 2005;10(3):505-20.

\section{Leonardi 2008}

Leonardi A, Motterle L, Bortolotti M. Allergy and the eye. Clinical and Experimental Immunology 2008;153(Suppl 1): 17-21.

Ono 2005

Ono SJ, Abelson MB. Allergic conjunctivitis: update on pathophysiology and prospects for future treatment. Journal of Allergy and Clinical Immunology 2005;115(1):118-22.

RevMan 2014

The Nordic Cochrane Centre, The Cochrane Collaboration. Review Manager (RevMan). 5.3. Copenhagen: The Nordic Cochrane Centre, The Cochrane Collaboration, 2014.

Simons 2002

Simons FE. Comparative pharmacology of $\mathrm{H} 1$ antihistamines: clinical relevance. American Journal of Medicine 2002;113(Suppl 9A):38-46.

Strachan 1997

Strachan D, Sibbald B, Weiland S, Ait-Khaled N, Anabwani $\mathrm{G}$, Anderson HR, et al. Worldwide variations in prevalence of symptoms of allergic rhinoconjunctivitis in children: the International Study of Asthma and Allergies in Childhood (ISAAC). Pediatric Allergy and Immunology 1997;8(4): 161-76.

Whitcup 2006

Whitcup SM, Cunningham ET Jr, Ng EW. Recent advances in ocular therapeutics. International Ophthalmology Clinics 2006;46(4):1-6.

\section{References to other published versions of this review}

\section{Mustafa 2012}

Mustafa MS, Castillo M, Mustafa MZ, Scott N, AzuaraBlanco A. Topical antihistamines for treating seasonal and perennial allergic conjunctivitis. Cochrane Database of Systematic Reviews 2012, Issue 1. [DOI: 10.1002/ 14651858.CD009566]

* Indicates the major publication for the study 


\section{CHARACTERISTICS OF STUDIES}

\section{Characteristics of included studies [ordered by study ID]}

\section{Avunduk 2005}

\begin{tabular}{ll}
\hline Methods & Parallel-group RCT \\
\hline Participants & 49 participants recruited with seasonal allergic conjunctivitis \\
\hline Interventions & $\begin{array}{l}\text { Three treatment arms: ketotifen } 0.025 \% \text { ophthalmic solution; olopatadine hydrochlo- } \\
\text { ride } 0.1 \% \text { ophthalmic solution; artificial tear substitute (preservative free). Duration of } \\
\text { treatment } 30 \text { days, follow-up } 30 \text { days (2-month study) }\end{array}$ \\
\hline Outcomes & $\begin{array}{l}\text { Participant evaluation of ocular symptoms (itching, tearing) } \\
\text { Investigator assessment of ocular signs (redness, eyelid swelling, chemosis) } \\
\text { Mean scores (using a scale range } 0-3 ; 0=\text { none, } 3=\text { severe) } \\
\text { Time points: at day } 0,15, \text { and } 30 \text { of treatment }\end{array}$ \\
\hline Country & Turkey \\
\hline
\end{tabular}

Number randomised, gender (male:fe- 39 participants randomised. M:F 20:19 male)

\begin{tabular}{l|l} 
Age mean (SD), median, range & Overall range 18-61 years \\
\hline Notes & $\begin{array}{l}\text { Study conducted from April to May 2004. Source of funding not stated. Declaration of } \\
\text { interest by the authors was not stated }\end{array}$ \\
\hline
\end{tabular}

\section{Risk of bias}

\begin{tabular}{lll}
\hline Bias & Authors' judgement & Support for judgement \\
\hline $\begin{array}{l}\text { Random sequence generation (selection } \\
\text { bias) }\end{array}$ & Low risk & $\begin{array}{l}\text { Computer-generated list (p.1394 - study medications): “Eligible } \\
\text { patients were randomly assigned, in a 1:1 ratio using a computer- } \\
\text { generated list of random numbers...” }\end{array}$ \\
\hline Allocation concealment (selection bias) & Unclear risk & $\begin{array}{l}\text { The method used to conceal the allocation sequence was not } \\
\text { described }\end{array}$ \\
\hline $\begin{array}{l}\text { Incomplete outcome data (attrition bias) } \\
\text { All outcomes }\end{array}$ & Low risk & $\begin{array}{l}\text { Data were nearly fully reported for each intervention group with } \\
20 \% \text { lost to follow-up (Figure 1 in the trial report), reasons were } \\
\text { not given. A similar number of participants were lost to follow- } \\
\text { up in each arm }\end{array}$ \\
\hline Selective reporting (reporting bias) & Low risk & Evidence was available of proper outcome reporting \\
\hline Other bias & Low risk & No apparent evidence of other risk of bias
\end{tabular}




\section{Avunduk 2005 (Continued)}

Blinding of participants and personnel Low risk (performance bias)

All outcomes

Blinding of outcome assessment (detection Low risk bias)

All outcomes
Masking of participants (p.1394 - study medications): "To maintain masking, all medications had identical packaging, color, consistency, $\mathrm{pH}$, and texture"

Investigators masked (p.1394 - efficacy assessments): “...ocular signs (redness, eyelid swelling, and chemosis) were graded, using slit-lamp examination and ordinal grading scales, by an investigator masked to treatment assignment."

\section{Azevedo 1991}

\begin{tabular}{ll}
\hline Methods & Parallel-group RCT \\
\hline Participants & 63 participants recruited with moderate or severe allergic conjunctivitis \\
\hline Interventions & $\begin{array}{l}\text { Three treatment arms: levocabastine } 0.5 \mathrm{mg} / \mathrm{ml} \text { eye drops; sodium cromoglycate } 20 \mathrm{mg} / \\
\mathrm{ml} \text { eye drops; placebo eye drops. Duration of treatment } 28 \text { days }\end{array}$ \\
\hline Outcomes & $\begin{array}{l}\text { Participant assessment (ocular irritation, redness, photophobia, tearing, swollen eyelids, } \\
\text { conjunctival oedema using a 4-point scale; } 0=\text { none, } 3=\text { severe) } \\
\text { Investigator assessment (ocular irritation, redness, photophobia, tearing, swollen eyelids, } \\
\text { conjunctival oedema, using a 4-point scale; } 0=\text { none, } 3=\text { severe) } \\
\text { Time points: at baseline and day } 14 \text { and } 28 \text { of treatment }\end{array}$ \\
\hline Country & Portugal \\
\hline
\end{tabular}

Number randomised, gender (male:fe- 63 participants randomised, 60 participants analysed. M:F 24:36 male)

Age mean (SD), median, range

Notes

Risk of bias

\section{Bias}

\section{Authors' judgement Support for judgement}

Random sequence generation (selection Low risk bias)

Allocation concealment (selection bias) Unclear risk
Median (range): levocabastine 27 years (13-55); sodium cromoglycate 26 years (9-46); placebo 34 years $(12-51)$

Not reported when study was conducted. Source of funding not reported. Declaration of interest by the authors was not stated 


\section{Azevedo 1991 (Continued)}

\begin{tabular}{l|l|l}
\hline $\begin{array}{l}\text { Incomplete outcome data (attrition bias) } \\
\text { All outcomes }\end{array}$ & Low risk & $\begin{array}{l}\text { Data were nearly fully reported, with less than 10\% lost to fol- } \\
\text { low-up, and the reasons were given }\end{array}$ \\
\hline $\begin{array}{l}\text { Selective reporting (reporting bias) } \\
\text { Other bias }\end{array}$ & Low risk & Low risk \\
\hline $\begin{array}{l}\text { Blinding of participants and personnel } \\
\text { (performance bias) } \\
\text { All outcomes }\end{array}$ & Low risk & No apparent evidence of other risk of bias \\
\hline $\begin{array}{l}\text { Blinding of outcome assessment (detection } \\
\text { bias) } \\
\text { All outcomes }\end{array}$ & Low risk & $\begin{array}{l}\text { Double-masked (p.691 - treatment and methods): "The trial } \\
\text { featured a doubled-blind, parallel groups design" }\end{array}$ \\
\hline
\end{tabular}

\section{Canonica 2003}

\begin{tabular}{|c|c|}
\hline Methods & Parallel-group RCT \\
\hline Participants & 139 participants recruited with moderate to severe perennial allergic conjunctivitis \\
\hline Interventions & $\begin{array}{l}\text { Three treatment arms: azelastine } 0.05 \% \text { eye drops; placebo eye drops; levocabastine eye } \\
\text { drops. Duration of treatment } 6 \text { weeks }\end{array}$ \\
\hline Outcomes & $\begin{array}{l}\text { Investigator assessment (itching and redness). Change in clinical sum score (itching and } \\
\text { redness using a scale range } 0-6 \text { ) } \\
\text { Participant assessment of ocular symptoms (daily diaries using a 4-point scale; } 0=\text { none, } \\
3=\text { severe symptoms) } \\
\text { Time points: at baseline and day } 7,21 \text {, and } 42 \text { of treatment }\end{array}$ \\
\hline Country & France, Italy, Spain, Russia, and United Kingdom \\
\hline $\begin{array}{l}\text { Number randomised, gender (male:fe- } \\
\text { male) }\end{array}$ & 139 participants randomised. M:F 65:74 \\
\hline Age mean (SD), median, range & $\begin{array}{l}\text { Mean (SD): azelastine group } 34 \text { years (13.7); placebo group } 36 \text { years (13.0); levocabastine } \\
34 \text { years (13.2) }\end{array}$ \\
\hline Notes & $\begin{array}{l}\text { Study conducted from } 2 \text { December } 1998 \text { to } 16 \text { June } 1999 \text {. Source of funding: University } \\
\text { of Genoa, Italy and Clinical and Biometrical Development, VIATRIS GmbH \& Co. } \\
\text { KG. Declaration of interest by the authors was not stated }\end{array}$ \\
\hline
\end{tabular}

\section{Risk of bias}

\section{Bias}

\section{Authors' judgement Support for judgement}




\section{Canonica 2003 (Continued)}

\begin{tabular}{|c|c|c|}
\hline $\begin{array}{l}\text { Random sequence generation (selection } \\
\text { bias) }\end{array}$ & Low risk & $\begin{array}{l}\text { Randomisation code ( } \mathrm{p} .323 \text { - drugs): "A predefined randomisa- } \\
\text { tion code was used to assign azelastine, placebo or levocabastine } \\
\text { treatment to qualified patients in an unbalanced fashion (ratio } \\
2: 2: 1) \text {." }\end{array}$ \\
\hline Allocation concealment (selection bias) & Unclear risk & $\begin{array}{l}\text { The method used to conceal the allocation sequence was not } \\
\text { described }\end{array}$ \\
\hline $\begin{array}{l}\text { Incomplete outcome data (attrition bias) } \\
\text { All outcomes }\end{array}$ & Low risk & $\begin{array}{l}\text { Data were nearly fully reported, with less than } 10 \% \text { lost to fol- } \\
\text { low-up, and the reasons were given }\end{array}$ \\
\hline Selective reporting (reporting bias) & Low risk & Evidence was available of proper outcome reporting \\
\hline Other bias & Low risk & No apparent evidence of other risk of bias \\
\hline $\begin{array}{l}\text { Blinding of participants and personnel } \\
\text { (performance bias) } \\
\text { All outcomes }\end{array}$ & Low risk & $\begin{array}{l}\text { Double-masked (p.323 - drugs): "Both azelastine eye drops and } \\
\text { the matching vehicle containing placebo were provided by VI- } \\
\text { ATRIS GmbH in identical packaging." }\end{array}$ \\
\hline $\begin{array}{l}\text { Blinding of outcome assessment (detection } \\
\text { bias) } \\
\text { All outcomes }\end{array}$ & Low risk & $\begin{array}{l}\text { Double-masked (p.323 - drugs): "Both azelastine eye drops and } \\
\text { the matching vehicle containing placebo were provided by VI- } \\
\text { ATRIS GmbH in identical packaging." }\end{array}$ \\
\hline
\end{tabular}

\section{Carr 2013}

\begin{tabular}{ll}
\hline Methods & Parallel-group RCT \\
\hline Participants & 245 participants recruited with allergic conjunctivitis \\
\hline Interventions & $\begin{array}{l}\text { Two treatment arms: bepotastine besilate (BBOS) eye drops } 1.5 \% \text {; placebo eye drops. } \\
\text { Duration of treatment } 2 \text { weeks }\end{array}$ \\
\hline Outcomes & $\begin{array}{l}\text { Participant assessment of ocular itching (instantaneous and reflective) mean change scores } \\
\text { (using diaries and a 4-point scale; } 0=\text { none, } 3=\text { severe) } \\
\text { Time points: at baseline, day } 7 \text { and } 14 \text { after treatment }\end{array}$ \\
\hline Country & United States \\
\hline
\end{tabular}

Number randomised, gender (male:fe- 245 participants randomised. M:F 92:153 male)

Age mean (SD), median, range Mean (SD) BBOS group 38.7 (13.80) range 12-85; placebo group 41.6 (15.03) range $12-72$

Notes

Source of funding: ISTA Pharmacologicals. The main investigators declared they had no financial interests in the outcome of the clinical trial. Carr and Ratner also declared that they were speakers and consultants for ISTA and Meda Pharmaceuticals 


\section{Carr 2013 (Continued)}

\section{Risk of bias}

\begin{tabular}{|c|c|c|}
\hline Bias & Authors' judgement & Support for judgement \\
\hline $\begin{array}{l}\text { Random sequence generation (selection } \\
\text { bias) }\end{array}$ & Low risk & $\begin{array}{l}\text { Computer-generated randomisation list (p. } 249 \text { - clinical trial } \\
\text { design): "...were assigned to receive BBOS } 1.5 \% \text { or placebo in a } \\
1: 1 \text { ratio as determined by a computer-generated randomisation } \\
\text { list." }\end{array}$ \\
\hline Allocation concealment (selection bias) & Unclear risk & $\begin{array}{l}\text { The method used to conceal the allocation sequence was not } \\
\text { described }\end{array}$ \\
\hline $\begin{array}{l}\text { Incomplete outcome data (attrition bias) } \\
\text { All outcomes }\end{array}$ & Low risk & $\begin{array}{l}\text { Data were nearly fully reported, with less than } 10 \% \text { lost to } \\
\text { follow-up, and the reasons were given }\end{array}$ \\
\hline Selective reporting (reporting bias) & Low risk & Evidence was available of proper outcome reporting \\
\hline Other bias & Low risk & No apparent evidence of other risk of bias \\
\hline $\begin{array}{l}\text { Blinding of participants and personnel } \\
\text { (performance bias) } \\
\text { All outcomes }\end{array}$ & Low risk & $\begin{array}{l}\text { Masking of participants (p. } 249 \text { - clinical trial design): "Both test } \\
\text { agents were provided in identical packaging and manufactured } \\
\text { by Bausch \& Lomb (Tampa, FL)." }\end{array}$ \\
\hline $\begin{array}{l}\text { Blinding of outcome assessment (detection } \\
\text { bias) } \\
\text { All outcomes }\end{array}$ & Low risk & $\begin{array}{l}\text { Study conducted from July to October } 2010 \text {. Investigators mask- } \\
\text { ing (p. } 249 \text { - clinical trial design): "Subjects were instructed to } \\
\text { instill the double-masked test agent..." }\end{array}$ \\
\hline
\end{tabular}

\section{Davies 1993}

\begin{tabular}{ll}
\hline Methods & Parallel-group RCT \\
\hline Participants & 95 participants recruited with allergic conjunctivitis \\
\hline Interventions & $\begin{array}{l}\text { Three treatment arms: levocabastine } 0.5 \mathrm{mg} / \mathrm{ml} \text {; sodium cromoglycate } 20 \mathrm{mg} / \mathrm{ml} \text {; placebo } \\
\text { eye drops. Duration of treatment } 28 \text { days }\end{array}$ \\
\hline Outcomes & $\begin{array}{l}\text { Investigators' and participants' assessment of ocular symptoms (4-point qualitative scale: } \\
\text { excellent, good, moderate, poor) } \\
\text { Time points: at baseline, day } 14 \text { and } 28 \text { after treatment }\end{array}$ \\
\hline Country & United Kingdom \\
\hline
\end{tabular}

Number randomised, gender (male:fe- 95 participants randomised. M:F 40:49 male)

Age mean (SD), median, range

Mean (range) levocabastine 30 years (7-66); sodium cromoglycate 31 years (10-69); placebo 30 years (13-61) 


\section{Davies 1993 (Continued)}

$\begin{array}{ll}\text { Notes } & \text { Not reported when study was conducted. Source of funding not stated. Declaration of } \\ \text { interest by the authors was not stated }\end{array}$

\section{Risk of bias}

Bias

Authors' judgement Support for judgement

\begin{tabular}{|c|c|c|}
\hline $\begin{array}{l}\text { Random sequence generation (selection } \\
\text { bias) }\end{array}$ & Unclear risk & $\begin{array}{l}\text { The method used to generate the allocation sequence was not } \\
\text { described }\end{array}$ \\
\hline Allocation concealment (selection bias) & Unclear risk & $\begin{array}{l}\text { The method used to conceal the allocation sequence was not } \\
\text { described }\end{array}$ \\
\hline $\begin{array}{l}\text { Incomplete outcome data (attrition bias) } \\
\text { All outcomes }\end{array}$ & High risk & Percentages given (numerators/denominators unclear) \\
\hline Selective reporting (reporting bias) & High risk & $P$ values given for selected outcomes \\
\hline Other bias & Unclear risk & No apparent evidence of other risk of bias \\
\hline $\begin{array}{l}\text { Blinding of participants and personnel } \\
\text { (performance bias) } \\
\text { All outcomes }\end{array}$ & Low risk & $\begin{array}{l}\text { Double-masked (p.520 - methods): “...95 patients participated } \\
\text { in this double-blind, parallel group trial.” }\end{array}$ \\
\hline $\begin{array}{l}\text { Blinding of outcome assessment (detection } \\
\text { bias) } \\
\text { All outcomes }\end{array}$ & Low risk & $\begin{array}{l}\text { Double-masked (p.520 - methods): “...95 patients participated } \\
\text { in this double-blind, parallel group trial.” }\end{array}$ \\
\hline
\end{tabular}

Fujishima 2008

\begin{tabular}{|c|c|}
\hline Methods & Parallel-group RCT \\
\hline Participants & 32 participants recruited with seasonal and perennial allergic conjunctivitis \\
\hline Interventions & $\begin{array}{l}\text { Two treatment arms: levocabastine; levocabastine and pemirolast potassium. Duration } \\
\text { of treatment } 1 \text { week }\end{array}$ \\
\hline Outcomes & $\begin{array}{l}\text { Participants used a diary to quantify ocular symptoms (itching, foreign body sensation, } \\
\text { tearing, eye discharge, and hyperaemia) using visual analogue scale } \\
\text { Investigators used a } 4 \text {-point scale ( } 0=\text { none, } 3=\text { severe) for quantifying ocular signs: } \\
\text { conjunctival hyperaemia, conjunctival oedema, eyelid oedema, conjunctival papilla for- } \\
\text { mation, and superficial punctate keratopathy } \\
\text { Time points: at baseline and } 1 \text { week after treatment }\end{array}$ \\
\hline Country & Japan \\
\hline
\end{tabular}


Fujishima 2008 (Continued)

\begin{tabular}{l|l}
$\begin{array}{l}\text { Number randomised, gender (male:fe- } \\
\text { male) }\end{array}$ & 32 participants randomised, 30 participants analysed. M:F 9:23 \\
\hline Age mean (SD), median, range & $\begin{array}{l}\text { Mean (range): levocabastine } 50.9 \text { years (12-81); levocabastine and pemirolast potassium } \\
40.5 \text { years }(7-73)\end{array}$ \\
\hline Notes & $\begin{array}{l}\text { Not reported when study was conducted. Source of funding unclear. Declaration of } \\
\text { interest by the authors was not stated }\end{array}$ \\
\hline
\end{tabular}

\section{Risk of bias}

\section{Bias}

Random sequence generation (selection Low risk bias)

\begin{tabular}{|c|c|c|}
\hline Allocation concealment (selection bias) & Unclear risk & $\begin{array}{l}\text { The method used to conceal the allocation sequence was not } \\
\text { described }\end{array}$ \\
\hline $\begin{array}{l}\text { Incomplete outcome data (attrition bias) } \\
\text { All outcomes }\end{array}$ & Low risk & Data of all participants randomised was analysed and reported \\
\hline Selective reporting (reporting bias) & Low risk & Evidence was available of proper outcome reporting \\
\hline Other bias & Low risk & No apparent evidence of other risk of bias \\
\hline $\begin{array}{l}\text { Blinding of participants and personnel } \\
\text { (performance bias) } \\
\text { All outcomes }\end{array}$ & Unclear risk & $\begin{array}{l}\text { The study did not describe details on masking of participants } \\
\text { on the intervention allocations }\end{array}$ \\
\hline $\begin{array}{l}\text { Blinding of outcome assessment (detection } \\
\text { bias) } \\
\text { All outcomes }\end{array}$ & Unclear risk & $\begin{array}{l}\text { The study did not describe details on masking of personnel/ } \\
\text { investigators on the intervention allocations }\end{array}$ \\
\hline
\end{tabular}

\section{Giede-Tuch 1998}

\begin{tabular}{ll}
\hline Methods & Parallel-group RCT \\
\hline Participants & $\begin{array}{l}151 \text { participants with seasonal allergic conjunctivitis or rhinoconjunctivitis for at least } 1 \\
\text { year }\end{array}$ \\
\hline Interventions & $\begin{array}{l}\text { Three treatment arms: azelastine } 0.025 \% \text {; azelastine } 0.05 \% \text {; placebo. Duration of treat- } \\
\text { ment } 14 \text { days }\end{array}$ \\
\hline Outcomes & $\begin{array}{l}\text { Participant-assessed itching, tearing, and redness. Sum score (itching, tearing, redness) } \\
\text { and rates of decreased scores by at least } 3 \text { score points between day 0-3 using a 4-point } \\
\text { scale }(0=\text { none, } 3=\text { severe }) \text { were used }\end{array}$
\end{tabular}

Topical antihistamines and mast cell stabilisers for treating seasonal and perennial allergic conjunctivitis (Review)

Sealed-envelope technique (p.233 - test agents): "The patients were randomly allocated to one of two groups by a sealed-enveloped technique...”

The method used to conceal the allocation sequence was not .

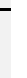


Giede-Tuch 1998 (Continued)

Investigator-assessed itching, tearing, and redness. A composite sum symptom mean score (itching, tearing, redness) using a 4-point scale $(0=$ none, $3=$ severe $)$ was analysed Time points: at baseline, day 3, 7, and 14 after treatment

Country Germany

Number randomised, gender (male:fe- 151 participants randomised, 129 participants analysed. M:F 66:85 male)

Age mean (SD), median, range
Mean (SD) azelastine $0.025 \% 35.4$ years (11.4); azelastine $0.05 \% 35.2$ years (10.7); placebo 35.9 years $(11.5)$

Study conducted from March to September 1994. Source of funding not stated. Declaration of interest by the authors was not stated

\section{Risk of bias}

\begin{tabular}{|c|c|c|}
\hline Bias & Authors' judgement & Support for judgement \\
\hline $\begin{array}{l}\text { Random sequence generation (selection } \\
\text { bias) }\end{array}$ & Unclear risk & $\begin{array}{l}\text { The method used to generate the allocation sequence was not } \\
\text { described }\end{array}$ \\
\hline Allocation concealment (selection bias) & Unclear risk & $\begin{array}{l}\text { The method used to conceal the allocation sequence was not } \\
\text { described }\end{array}$ \\
\hline $\begin{array}{l}\text { Incomplete outcome data (attrition bias) } \\
\text { All outcomes }\end{array}$ & Low risk & $\begin{array}{l}\text { Data were nearly fully reported, with less than } 15 \% \text { lost to } \\
\text { follow-up, and reasons were given }\end{array}$ \\
\hline Selective reporting (reporting bias) & Low risk & Evidence was available of proper outcome reporting \\
\hline Other bias & Low risk & No apparent evidence of other risk of bias \\
\hline $\begin{array}{l}\text { Blinding of participants and personnel } \\
\text { (performance bias) } \\
\text { All outcomes }\end{array}$ & Low risk & $\begin{array}{l}\text { Double-masked (p. } 858 \text { - study design): "The investigation was } \\
\text { performed as a double-blind, randomized, placebo-controlled } \\
\text { study..." }\end{array}$ \\
\hline $\begin{array}{l}\text { Blinding of outcome assessment (detection } \\
\text { bias) } \\
\text { All outcomes }\end{array}$ & Low risk & $\begin{array}{l}\text { Double-masked (p. } 858 \text { - study design): "The investigation was } \\
\text { performed as a double-blind, randomized, placebo-controlled } \\
\text { study..." }\end{array}$ \\
\hline
\end{tabular}

\section{Graue 1994}

Methods

Participants

Interventions
Parallel-group RCT

Participants from 5 to 20 years old with seasonal conjunctivitis

Two treatment arms: levocabastine $0.5 \mathrm{mg} / \mathrm{ml}$; placebo. Duration of treatment 7 days 


\section{Graue 1994 (Continued)}

\begin{tabular}{ll}
\hline Outcomes & $\begin{array}{l}\text { Participants' assessment of ocular symptoms (visual analogue scale; } 0=\text { none, } 10=\text { worse } \\
\text { possible) } \\
\text { Investigator-evaluated symptoms (absent, mild, moderate, and severe) } \\
\text { Time points: at baseline and day } 7 \text { after treatment }\end{array}$ \\
\hline Country & Mexico \\
\hline
\end{tabular}

Number randomised, gender (male:fe- 40 participants randomised. M:F 30:10 male)

Age mean (SD), median, range

Notes
Age mean: levocabastine 9.1 years; placebo 10.1 years

Not reported when study was conducted. Source of funding not stated. Declaration of interest by the authors was not stated

\section{Risk of bias}

\begin{tabular}{|c|c|c|}
\hline Bias & Authors' judgement & Support for judgement \\
\hline $\begin{array}{l}\text { Random sequence generation (selection } \\
\text { bias) }\end{array}$ & Unclear risk & $\begin{array}{l}\text { The method used to generate the allocation sequence was not } \\
\text { described (p. } 37 \text { - material and methods): "patients were divided } \\
\text { randomly into two groups..." }\end{array}$ \\
\hline Allocation concealment (selection bias) & Unclear risk & $\begin{array}{l}\text { The method used to conceal the allocation sequence was not } \\
\text { described }\end{array}$ \\
\hline $\begin{array}{l}\text { Incomplete outcome data (attrition bias) } \\
\text { All outcomes }\end{array}$ & Low risk & $\begin{array}{l}\text { Data were nearly fully reported, with less than } 10 \% \text { lost to fol- } \\
\text { low-up, and reasons were given }\end{array}$ \\
\hline Selective reporting (reporting bias) & Low risk & Evidence was available of proper outcome reporting \\
\hline Other bias & Low risk & No apparent evidence of other risk of bias \\
\hline $\begin{array}{l}\text { Blinding of participants and personnel } \\
\text { (performance bias) } \\
\text { All outcomes }\end{array}$ & Low risk & $\begin{array}{l}\text { Double-masked (p. } 37 \text { - material and methods): "The study de- } \\
\text { sign is double-blind, prospective and comparative..." } \\
\text { "both drugs were provided by Janssen Pharmaceutical in similar } \\
\text { plastic bottles of } 4 \mathrm{ml} \text { each, labeled as levocabastine (including } \\
\text { the ones that contained placebo) and with an identification code } \\
\text { number..." }\end{array}$ \\
\hline $\begin{array}{l}\text { Blinding of outcome assessment (detection } \\
\text { bias) } \\
\text { All outcomes }\end{array}$ & Low risk & $\begin{array}{l}\text { Double-masked (p. } 37 \text { - material and methods): "The study de- } \\
\text { sign is double-blind, prospective and comparative..." } \\
\text { "both drugs were provided by Janssen Pharmaceutical in similar } \\
\text { plastic bottles of } 4 \mathrm{ml} \text { each, labeled as levocabastine (including } \\
\text { the ones that contained placebo) and with an identification code } \\
\text { number..." }\end{array}$ \\
\hline
\end{tabular}


Hechanova 1984

\begin{tabular}{|c|c|c|}
\hline Methods & \multicolumn{2}{|l|}{ Parallel-group RCT } \\
\hline Participants & \multicolumn{2}{|c|}{40 participants with allergic conjunctivitis } \\
\hline Interventions & \multicolumn{2}{|c|}{ Two treatment arms: sodium cromoglycate; placebo. Duration of treatment 4 weeks } \\
\hline Outcomes & \multicolumn{2}{|c|}{$\begin{array}{l}\text { Participants' and clinicians' assessment of ocular symptoms and opinion of efficacy of } \\
\text { treatment: specifically they assessed itching, soreness, redness, grittiness, photophobia } \\
\text { using a 5-point scale ( } 0=\text { none, } 5=\text { very severe) } \\
\text { Time points: at } 1,2 \text {, and } 4 \text { weeks after treatment } \\
\text { Results of photophobia were not reported }\end{array}$} \\
\hline Country & \multicolumn{2}{|l|}{ Philippines } \\
\hline $\begin{array}{l}\text { Number randomised, gender (male:fe- } \\
\text { male) }\end{array}$ & \multicolumn{2}{|c|}{40 participants randomised. M:F 20:20 } \\
\hline Age mean (SD), median, range & \multicolumn{2}{|c|}{ Mean (range) 35 years $(5-71)$} \\
\hline Notes & \multicolumn{2}{|c|}{$\begin{array}{l}\text { Not reported when study was conducted. Source of funding not stated. Declaration of } \\
\text { interest by the authors was not stated }\end{array}$} \\
\hline \multicolumn{3}{|l|}{ Risk of bias } \\
\hline Bias & Authors' judgement & Support for judgement \\
\hline $\begin{array}{l}\text { Random sequence generation (selection } \\
\text { bias) }\end{array}$ & Unclear risk & $\begin{array}{l}\text { The method used to generate the allocation sequence was not } \\
\text { described (p. } 60 \text { - material and methods): “...with patients ran- } \\
\text { domly allocated to receive...” }\end{array}$ \\
\hline Allocation concealment (selection bias) & Unclear risk & $\begin{array}{l}\text { The method used to conceal the allocation sequence was not } \\
\text { described }\end{array}$ \\
\hline $\begin{array}{l}\text { Incomplete outcome data (attrition bias) } \\
\text { All outcomes }\end{array}$ & High risk & High rates of withdrawals \\
\hline Selective reporting (reporting bias) & High risk & Only selected results were presented \\
\hline Other bias & Low risk & No apparent evidence of other risk of bias \\
\hline $\begin{array}{l}\text { Blinding of participants and personnel } \\
\text { (performance bias) } \\
\text { All outcomes }\end{array}$ & Low risk & $\begin{array}{l}\text { Double-masked (p.60 - material and methods): "The trial was } \\
\text { a double-blind group comparison..." }\end{array}$ \\
\hline $\begin{array}{l}\text { Blinding of outcome assessment (detection } \\
\text { bias) } \\
\text { All outcomes }\end{array}$ & Low risk & $\begin{array}{l}\text { Double-masked (p.60 -material and methods): "The trial was a } \\
\text { double-blind group comparison..." }\end{array}$ \\
\hline
\end{tabular}

Topical antihistamines and mast cell stabilisers for treating seasonal and perennial allergic conjunctivitis (Review) 
Höffling-Lima 2001

\begin{tabular}{|c|c|}
\hline Methods & Parallel-group RCT \\
\hline Participants & 40 participants with allergic conjunctivitis \\
\hline Interventions & Two treatment arms: ketotifen $0.05 \%$; olopatadine $0.1 \%$. Duration of treatment 30 days \\
\hline Outcomes & $\begin{array}{l}\text { Participants' assessment of severity of ocular symptoms: itching, burning, watering, dis- } \\
\text { charge, photophobia ( } 4 \text {-point scale; } 0=\text { none, } 3=\text { severe) } \\
\text { Investigator assessment of ocular signs } \\
\text { Time points: at days } 1,2,7,14 \text {, and } 30 \text { after treatment }\end{array}$ \\
\hline Country & Brazil \\
\hline $\begin{array}{l}\text { Number randomised, gender (male:fe- } \\
\text { male) }\end{array}$ & 40 participants randomised, 34 participants analysed. M:F 15:19 \\
\hline Age mean (SD), median, range & Mean (range) 27 years $(5-73)$ \\
\hline Notes & $\begin{array}{l}\text { Study conducted from } 1 \text { February to } 30 \text { June } 1999 \text {. Source of funding not stated. The } \\
\text { authors declared no commercial interest in the drugs evaluated and did not receive any } \\
\text { direct or indirect benefit or financial support for this study }\end{array}$ \\
\hline
\end{tabular}

\section{Risk of bias}

\begin{tabular}{|c|c|c|}
\hline Bias & Authors' judgement & Support for judgement \\
\hline $\begin{array}{l}\text { Random sequence generation (selection } \\
\text { bias) }\end{array}$ & Low risk & $\begin{array}{l}\text { Randomisation (p. } 416 \text { - methods): "A masked and randomised } \\
\text { clinical study was conducted..." } \\
\text { "the introduction of treatment was randomized beforehand..." }\end{array}$ \\
\hline Allocation concealment (selection bias) & Unclear risk & $\begin{array}{l}\text { The method used to conceal the allocation sequence was not } \\
\text { described }\end{array}$ \\
\hline $\begin{array}{l}\text { Incomplete outcome data (attrition bias) } \\
\text { All outcomes }\end{array}$ & Low risk & $\begin{array}{l}\text { Data were nearly fully reported, with } 15 \% \text { lost to follow-up, and } \\
\text { reasons were given }\end{array}$ \\
\hline Selective reporting (reporting bias) & Low risk & Evidence was available of proper outcome reporting \\
\hline Other bias & Low risk & No apparent evidence of other risk of bias \\
\hline $\begin{array}{l}\text { Blinding of participants and personnel } \\
\text { (performance bias) } \\
\text { All outcomes }\end{array}$ & Low risk & $\begin{array}{l}\text { Masking of participants (p. } 416 \text { - methods): "A masked and ran- } \\
\text { domised clinical study was conducted..." } \\
\text { "... and bottles with topical medications were masked by oph- } \\
\text { thalmos laboratory." }\end{array}$ \\
\hline $\begin{array}{l}\text { Blinding of outcome assessment (detection } \\
\text { bias) } \\
\text { All outcomes }\end{array}$ & Low risk & $\begin{array}{l}\text { Masking of investigator (p. } 417 \text { - methods): "only the medical } \\
\text { monitors delivered the drugs to patients..." }\end{array}$ \\
\hline
\end{tabular}

Topical antihistamines and mast cell stabilisers for treating seasonal and perennial allergic conjunctivitis (Review)

Copyright @ 2015 The Cochrane Collaboration. Published by John Wiley \& Sons, Ltd. 
James 2003

\begin{tabular}{|c|c|c|}
\hline Methods & \multicolumn{2}{|l|}{ Parallel-group RCT } \\
\hline Participants & \multicolumn{2}{|c|}{144 participants with seasonal allergic conjunctivitis } \\
\hline Interventions & \multicolumn{2}{|c|}{$\begin{array}{l}\text { Three treatment arms: azelastine; sodium cromoglycate; placebo. Duration of treatment } \\
14 \text { days }\end{array}$} \\
\hline Outcomes & \multicolumn{2}{|c|}{$\begin{array}{l}\text { Participants' assessment of symptoms (composite score): itching, redness, tearing, foreign } \\
\text { body sensation, photophobia, soreness, discharge (range 0-9) using participant's diary } \\
\text { during treatment ( } 14 \text { days) } \\
\text { Investigator assessment of itching, tearing, conjunctival redness (range 0-9, composite } \\
\text { sum score) } \\
\text { Time points: at day } 3,7 \text {, and } 14 \text { after treatment }\end{array}$} \\
\hline Country & \multicolumn{2}{|c|}{ Germany and United Kingdom } \\
\hline $\begin{array}{l}\text { Number randomised, gender (male:fe- } \\
\text { male) }\end{array}$ & \multicolumn{2}{|c|}{144 participants randomised, 136 participants analysed. M:F 51:93 } \\
\hline Age mean (SD), median, range & \multicolumn{2}{|c|}{$\begin{array}{l}\text { Mean (range): azelastine } 37.1 \text { years (16-65); sodium cromoglycate } 35.5 \text { years (18-65); } \\
\text { placebo } 35.8 \text { years }(18-64)\end{array}$} \\
\hline Notes & \multicolumn{2}{|c|}{$\begin{array}{l}\text { Study conducted from April to September 1995. Source of funding: ASTA Medica AG } \\
\text { (now VIATRIS GmbH \& Co. KG). Declaration of interest by the authors was not stated }\end{array}$} \\
\hline \multicolumn{3}{|l|}{ Risk of bias } \\
\hline Bias & Authors' judgement & Support for judgement \\
\hline $\begin{array}{l}\text { Random sequence generation (selection } \\
\text { bias) }\end{array}$ & Unclear risk & $\begin{array}{l}\text { The method used to generate the allocation sequence was not } \\
\text { described }\end{array}$ \\
\hline Allocation concealment (selection bias) & Unclear risk & $\begin{array}{l}\text { The method used to conceal the allocation sequence was not } \\
\text { described }\end{array}$ \\
\hline $\begin{array}{l}\text { Incomplete outcome data (attrition bias) } \\
\text { All outcomes }\end{array}$ & Low risk & $\begin{array}{l}\text { Data were nearly fully reported, with less than } 10 \% \text { lost to fol- } \\
\text { low-up, and reasons were given }\end{array}$ \\
\hline Selective reporting (reporting bias) & Low risk & Evidence was available of proper outcome reporting \\
\hline Other bias & Low risk & No apparent evidence of other risk of bias \\
\hline $\begin{array}{l}\text { Blinding of participants and personnel } \\
\text { (performance bias) } \\
\text { All outcomes }\end{array}$ & Unclear risk & $\begin{array}{l}\text { Partial double-masked (p.314 - study design): "partial double- } \\
\text { blind, parallel-group... The appearance and application regimen } \\
\text { of placebo eye drops was identical to azelastine and the study } \\
\text { was double-blind in this respect." }\end{array}$ \\
\hline
\end{tabular}

Topical antihistamines and mast cell stabilisers for treating seasonal and perennial allergic conjunctivitis (Review) 
James 2003 (Continued)

Blinding of outcome assessment (detection Unclear risk bias)

All outcomes
Partial double-masked (p.314 - study design): "However, the study was open in respect of sodium cromoglycate-treated patients where the bottle size and dosage regimen identified this treatment to the investigator."

Katelaris 2002

\begin{tabular}{ll}
\hline Methods & Parallel-group RCT \\
\hline Participants & 188 participants with seasonal allergic conjunctivitis \\
\hline Interventions & $\begin{array}{l}\text { Two treatment arms: olopatadine hydrochloride 0.1\%; cromolyn sodium 2\% (ne- } \\
\text { docromil sodium). Duration of treatment } 6 \text { weeks }\end{array}$ \\
\hline Outcomes & $\begin{array}{l}\text { Investigator's assessment of ocular signs and symptoms: mean ocular symptom scores of } \\
\text { itching and redness and physicians' impression scale scores, per protocol data }(5 \text {-point } \\
\text { scale; } 0=\text { none, } 4 \text { = very frequent) } \\
\text { Participants' assessment of itching and redness using diary (10-point scale; } 0=\text { none, } 9 \\
=\text { severe) } \\
\text { Time points: at days } 1 \text { to } 14 \text {, and } 30 \text { to } 42 \text { after treatment }\end{array}$ \\
\hline Country & \begin{tabular}{l}
6 European countries and Australia \\
\hline
\end{tabular}
\end{tabular}

Number randomised, gender (male:fe- 188 participants randomised, 185 participants analysed. M:F 103:82 male)

Age mean (SD), median, range

Notes
Mean (SD): olopatadine 33.0 years (19.3); cromolyn sodium 36.8 years (20.9)

Not reported when study was conducted. Source of funding not stated. Declaration of interest by the authors was not stated

\section{Risk of bias}

\section{Bias}

Random sequence generation (selection Low risk bias)

\begin{tabular}{|c|c|c|}
\hline Allocation concealment (selection bias) & Unclear risk & $\begin{array}{l}\text { The method used to conceal the allocation sequence was not } \\
\text { described }\end{array}$ \\
\hline
\end{tabular}

Incomplete outcome data (attrition bias) Unclear risk All outcomes

Selective reporting (reporting bias)

Low risk
Analysis per-protocol participants not intention-to-treat analysis. Less than $10 \%$ lost to follow-up, and reasons were given

Evidence was available of adequate outcome reporting 
Katelaris 2002 (Continued)

\begin{tabular}{|c|c|c|}
\hline Other bias & Low risk & No apparent evidence of other risk of bias \\
\hline $\begin{array}{l}\text { Blinding of participants and personnel } \\
\text { (performance bias) } \\
\text { All outcomes }\end{array}$ & Low risk & $\begin{array}{l}\text { Double-masking (p. } 1563 \text { - study procedures): "Double-masking } \\
\text { was ensured through the use of identical opaque bottles and } \\
\text { similar-appearing contents and labeling." }\end{array}$ \\
\hline $\begin{array}{l}\text { Blinding of outcome assessment (detection } \\
\text { bias) } \\
\text { All outcomes }\end{array}$ & Low risk & $\begin{array}{l}\text { Double masking (p. } 1563 \text { - study procedures): "Double-masking } \\
\text { was ensured through the use of identical opaque bottles and } \\
\text { similar-appearing contents and labeling." }\end{array}$ \\
\hline
\end{tabular}

Kidd 2003

\begin{tabular}{l|l}
\hline Methods & Parallel-group RCT \\
\hline Participants & 519 participants with seasonal allergic conjunctivitis \\
\hline Interventions & $\begin{array}{l}\text { Three treatment arms: ketotifen fumarate } 0.025 \% \text {; levocabastine } 0.05 \% \text {; placebo. Du- } \\
\text { ration of treatment } 4 \text { weeks }\end{array}$ \\
\hline Outcomes & $\begin{array}{l}\text { Participant assessment (mean scores) of ocular itching (using a 5-point scale; } 0=\text { none, } \\
\text { - severe) and watering (scale } 0=\text { none, } 3=\text { severe) within the first } 4 \text { days of treatment } \\
\text { Investigator assessment of signs (redness, eyelid swelling, chemosis) after treatment (using } \\
\text { a 5-point scale; } 0=\text { none, } 4=\text { severe) }\end{array}$ \\
\hline Country & Australia \\
\hline
\end{tabular}

Number randomised, gender (male:fe- 519 participants randomised, 348 participants analysed. M:F 269:254 male)

Age mean (SD), median, range

Mean (SD): ketotifen 46.3 years (17.0); levocabastine 49.5 years (17.4); placebo 47.9 years (17.4)

Notes

Not reported when study was conducted. Source of funding Novartis. Declaration of interest by the authors was not stated

\section{Risk of bias}

\begin{tabular}{ll|l}
\hline Bias & Authors' judgement & Support for judgement \\
\hline $\begin{array}{l}\text { Random sequence generation (selection } \\
\text { bias) }\end{array}$ & Low risk & $\begin{array}{l}\text { RANCODE software (p.1207 - study design): "Eligible subjects } \\
\text { were randomised (RANCODE version 3.6) to one of the three } \\
\text { treatment groups..." }\end{array}$ \\
\hline Allocation concealment (selection bias) & Unclear risk & $\begin{array}{l}\text { The method used to conceal the allocation sequence was not } \\
\text { described }\end{array}$
\end{tabular}

Topical antihistamines and mast cell stabilisers for treating seasonal and perennial allergic conjunctivitis (Review) 
Kidd 2003 (Continued)

\begin{tabular}{l|l|l}
$\begin{array}{l}\text { Incomplete outcome data (attrition bias) } \\
\text { All outcomes }\end{array}$ & Unclear risk & $\begin{array}{l}\text { Not intention-to-treat analysis, no reasons reported per protocol } \\
\text { deviations (large numbers). Number of participants randomised } \\
(519) \text { does not match the sum of male and female (523) }\end{array}$ \\
\hline $\begin{array}{l}\text { Selective reporting (reporting bias) } \\
\text { Other bias }\end{array}$ & Low risk & Lvidence was available of adequate outcome reporting. \\
\hline $\begin{array}{l}\text { Blinding of participants and personnel } \\
\text { (performance bias) } \\
\text { All outcomes }\end{array}$ & Low risk & No apparent evidence of other risk of bias \\
\hline
\end{tabular}

Blinding of outcome assessment (detection Low risk bias)

Identical packing (p.1207 - study design): “The packaging of all

All outcomes

\section{Lanier 2001}

\begin{tabular}{ll}
\hline Methods & Parallel-group RCT \\
\hline Participants & 94 participants with seasonal allergic conjunctivitis \\
\hline Interventions & $\begin{array}{l}\text { Two treatment arms: olopatadine and oral loratadine; oral loratadine. Duration of treat- } \\
\text { ment } 1 \text { week }\end{array}$ \\
\hline Outcomes & $\begin{array}{l}\text { Participants' and physicians' assessment of ocular signs and symptoms } \\
\text { Mean scores for ocular itching and redness using diaries and a 4-point scale } \\
\text { Time points: at baseline, day 3 and } 7 \text { after treatment }\end{array}$ \\
\hline Country & United States \\
\hline
\end{tabular}

Number randomised, gender (male:fe- 94 participants randomised, 72 participants analysed. M:F 33:61 male)

Age mean (SD), median, range

Mean (range): olopatadine and oral loratadine 39 years (7-74); oral loratadine 37 years (9-74)

Notes

Study conducted from May to November 1998. Source of funding Alcon Pharmaceutical.

Declaration of interest by the authors was not stated

\section{Risk of bias}

\section{Bias}

Random sequence generation (selection Unclear risk bias)

\section{Authors' judgement Support for judgement}

The method used to generate the allocation sequence was not described 
Lanier 2001 (Continued)

\begin{tabular}{|c|c|c|}
\hline Allocation concealment (selection bias) & Unclear risk & $\begin{array}{l}\text { The method used to conceal the allocation sequence was not } \\
\text { described }\end{array}$ \\
\hline $\begin{array}{l}\text { Incomplete outcome data (attrition bias) } \\
\text { All outcomes }\end{array}$ & Unclear risk & $\begin{array}{l}\text { Exclusion of } 22 \text { participants after randomisation (p.644 - re- } \\
\text { sults): "Ten patients were not evaluable for efficacy because they } \\
\text { did not meet inclusion/exclusion criteria or had no follow up } \\
\text { examination... Twelve patients exited the study prematurely..." }\end{array}$ \\
\hline Selective reporting (reporting bias) & Low risk & Evidence was available of proper outcome reporting \\
\hline Other bias & Low risk & No apparent evidence of other risk of bias \\
\hline $\begin{array}{l}\text { Blinding of participants and personnel } \\
\text { (performance bias) } \\
\text { All outcomes }\end{array}$ & High risk & $\begin{array}{l}\text { (p.643 - study design): "Because there were no control eye drops, } \\
\text { patients were aware of their treatment groups, but clinicians } \\
\text { performing the evaluations in the clinic were not." }\end{array}$ \\
\hline $\begin{array}{l}\text { Blinding of outcome assessment (detection } \\
\text { bias) } \\
\text { All outcomes }\end{array}$ & High risk & $\begin{array}{l}\text { (p.643 - study design): "Because there were no control eye drops, } \\
\text { patients were aware of their treatment groups, but clinicians } \\
\text { performing the evaluations in the clinic were not." }\end{array}$ \\
\hline
\end{tabular}

\section{Leino 1992}

\begin{tabular}{ll}
\hline Methods & Parallel-group RCT \\
\hline Participants & 195 participants with seasonal allergic conjunctivitis \\
\hline Interventions & $\begin{array}{l}\text { Three treatment arms: nedocromil sodium 2\%; sodium cromoglycate 2\%; placebo. Du- } \\
\text { ration of treatment 4 weeks }\end{array}$ \\
\hline Outcomes & Overall assessment by participants and investigators using a 4-point scale \\
\hline Country & Finland \\
\hline
\end{tabular}

Number randomised, gender (male:fe- 195 participants randomised, 185 participants analysed. M:F not stated male)

Age mean (SD), median, range Mean: nedocromil 20.8 years; sodium cromoglycate 19.3 years; placebo 19.7 years

Notes Not reported when study was conducted. Source of funding not stated. Declaration of interest by the authors was not stated

\section{Risk of bias}

\section{Bias}

Random sequence generation (selection Unclear risk bias)

\section{Authors' judgement Support for judgement}

The method used to conceal the allocation sequence was not described 
Leino 1992 (Continued)

\begin{tabular}{|c|c|c|}
\hline Allocation concealment (selection bias) & Low risk & $\begin{array}{l}\text { (p. } 930 \text { - material and methods): "After } 1-2 \text { weeks the patients } \\
\text { were issued their appropriate eye drops by randomized code } \\
\text { number..." }\end{array}$ \\
\hline $\begin{array}{l}\text { Incomplete outcome data (attrition bias) } \\
\text { All outcomes }\end{array}$ & High risk & $\begin{array}{l}\text { Withdrawal/exclusion of participants (p. } 931 \text { - results): "Data } \\
\text { were returned from } 195 \text { patients, } 10 \text { of whom withdrew without } \\
\text { taking test treatment and were excluded." } \\
\text { "A further } 12 \text { patients ( } 5 \text { nedocromil sodium, } 3 \text { sodium cromo- } \\
\text { glycate and } 4 \text { placebo) withdrew from the study without com- } \\
\text { pleting the 4-week treatment period." }\end{array}$ \\
\hline Selective reporting (reporting bias) & Low risk & Evidence was available of proper outcome reporting \\
\hline Other bias & Low risk & No apparent evidence of other risk of bias \\
\hline $\begin{array}{l}\text { Blinding of participants and personnel } \\
\text { (performance bias) } \\
\text { All outcomes }\end{array}$ & Low risk & $\begin{array}{l}\text { Identical drugs packaging (p. } 930 \text { - medication): "All test drugs } \\
\text { were supplied in identical } 10 \mathrm{ml} \text { plastic bottles." }\end{array}$ \\
\hline $\begin{array}{l}\text { Blinding of outcome assessment (detection } \\
\text { bias) } \\
\text { All outcomes }\end{array}$ & Low risk & $\begin{array}{l}\text { Identical drugs packaging (p. } 930 \text { - medication): "All test drugs } \\
\text { were supplied in identical } 10 \mathrm{ml} \text { plastic bottles." }\end{array}$ \\
\hline
\end{tabular}

\section{Lenhard 1997}

\begin{tabular}{|c|c|}
\hline Methods & Parallel-group RCT \\
\hline Participants & 278 participants with seasonal allergic conjunctivitis or rhinoconjunctivitis \\
\hline Interventions & $\begin{array}{l}\text { Three treatment arms: azelastine } 0.025 \% \text {; azelastine } 0.05 \% \text {; placebo. Duration of treat- } \\
\text { ment } 14 \text { days }\end{array}$ \\
\hline Outcomes & $\begin{array}{l}\text { Investigator mean score of } 3 \text { symptoms (itching, tearing, and redness (secondary analysis) } \\
\text { ) using a 4-point scale } \\
\text { Participants' assessment of symptoms (itching primary variable) using diaries and a 4- } \\
\text { point scale } \\
\text { Time points: at baseline, at treatment day } 7 \text { and } 14\end{array}$ \\
\hline Country & France, Italy, Poland, and Slovenia \\
\hline $\begin{array}{l}\text { Number randomised, gender (male:fe- } \\
\text { male) }\end{array}$ & 278 participants randomised, 226 participants analysed as per protocol. M:F 114:164 \\
\hline
\end{tabular}

Age mean (SD), median, range

Mean (SD) azelastine $0.025 \% 31.6$ years (10.6); azelastine $0.05 \% 31.7$ years (11.7); placebo 33.9 years (11.9)

Topical antihistamines and mast cell stabilisers for treating seasonal and perennial allergic conjunctivitis (Review) 
Lenhard 1997 (Continued)

\begin{tabular}{|c|c|c|}
\hline Notes & \multicolumn{2}{|c|}{$\begin{array}{l}\text { Not reported when study was conducted. Source of funding ASTA Medica AG. Decla- } \\
\text { ration of interest by the authors was not stated }\end{array}$} \\
\hline \multicolumn{3}{|l|}{ Risk of bias } \\
\hline Bias & Authors' judgement & Support for judgement \\
\hline $\begin{array}{l}\text { Random sequence generation (selection } \\
\text { bias) }\end{array}$ & Unclear risk & $\begin{array}{l}\text { The method used to generate the allocation sequence was not } \\
\text { described }\end{array}$ \\
\hline Allocation concealment (selection bias) & Unclear risk & $\begin{array}{l}\text { The method used to conceal the allocation sequence was not } \\
\text { described }\end{array}$ \\
\hline $\begin{array}{l}\text { Incomplete outcome data (attrition bias) } \\
\text { All outcomes }\end{array}$ & Unclear risk & $\begin{array}{l}\text { Of } 278 \text { recruited participants (p. } 24 \text { - results): " } 226 \text { patients com- } \\
\text { pleted the study as planned..." No reasons were given. (p. } 24 \text { - } \\
\text { results): "Twenty-four of the } 278 \text { recruited patients discontin- } \\
\text { ued the study prematurely." }\end{array}$ \\
\hline Selective reporting (reporting bias) & Low risk & Evidence was available of proper outcome reporting \\
\hline Other bias & Low risk & No apparent evidence of other risk of bias \\
\hline $\begin{array}{l}\text { Blinding of participants and personnel } \\
\text { (performance bias) } \\
\text { All outcomes }\end{array}$ & Unclear risk & $\begin{array}{l}\text { The study did not describe details on masking of participants } \\
\text { on the intervention allocations }\end{array}$ \\
\hline $\begin{array}{l}\text { Blinding of outcome assessment (detection } \\
\text { bias) } \\
\text { All outcomes }\end{array}$ & Unclear risk & $\begin{array}{l}\text {-The study did not describe details on masking of personnel/ } \\
\text { investigators on the intervention allocations }\end{array}$ \\
\hline
\end{tabular}

McCabe 2012

\begin{tabular}{ll}
\hline Methods & Parallel-group crossed-over RCT \\
\hline Participants & 30 participants recruited with allergic conjunctivitis \\
\hline Interventions & $\begin{array}{l}\text { Two treatment arms: bepotastine besilate } 1.5 \% \text { ophthalmic solution; olopatadine hy- } \\
\text { drochloride } 0.2 \%\end{array}$ \\
\hline Outcomes & $\begin{array}{l}\text { Participants' assessment of ocular itching (using a 5-point Likert scale; } 1=\text { lowest relief, } \\
5=\text { highest relief) at baseline and } 2 \text { other clinic visits but also using daily diary to assess } \\
\text { morning and evening ocular itch relief during } 2 \text { weeks of treatment }\end{array}$ \\
\hline Country & United States \\
\hline
\end{tabular}

Number randomised, gender (male:fe- 30 participants randomised, M:F 10:20 male)

Topical antihistamines and mast cell stabilisers for treating seasonal and perennial allergic conjunctivitis (Review)

Copyright @ 2015 The Cochrane Collaboration. Published by John Wiley \& Sons, Ltd. 
McCabe 2012 (Continued)

\begin{tabular}{|c|c|c|}
\hline Age mean $(\mathrm{SD})$, median, range & \multicolumn{2}{|c|}{ Mean (SD) 49.8 (2.76), range 23-75 } \\
\hline Notes & \multicolumn{2}{|c|}{$\begin{array}{l}\text { Study conducted in September 2011. Source of funding not stated. No authors have } \\
\text { financial or proprietary interest in any material or method mentioned in this work }\end{array}$} \\
\hline \multicolumn{3}{|l|}{ Risk of bias } \\
\hline Bias & Authors' judgement & Support for judgement \\
\hline $\begin{array}{l}\text { Random sequence generation (selection } \\
\text { bias) }\end{array}$ & Low risk & $\begin{array}{l}\text { Randomisation list (p.1733 - materials and meth- } \\
\text { ods): "The enrolled patients were assigned sequen- } \\
\text { tially according to a computer-generated random- } \\
\text { ization list..." }\end{array}$ \\
\hline Allocation concealment (selection bias) & Unclear risk & $\begin{array}{l}\text { The method used to conceal the allocation sequence } \\
\text { was not described }\end{array}$ \\
\hline $\begin{array}{l}\text { Incomplete outcome data (attrition bias) } \\
\text { All outcomes }\end{array}$ & Low risk & $\begin{array}{l}\text { Complete outcome data reported, all the partici- } \\
\text { pants randomised were analysed }\end{array}$ \\
\hline Selective reporting (reporting bias) & Low risk & Evidence was available of proper outcome reporting \\
\hline Other bias & Low risk & No apparent evidence of other risk of bias \\
\hline $\begin{array}{l}\text { Blinding of participants and personnel } \\
\text { (performance bias) } \\
\text { All outcomes }\end{array}$ & High risk & $\begin{array}{l}\text { Participants were not masked to treatment allocation } \\
\text { (p. } 1733 \text { - material and methods): "Each treatment } \\
\text { was provided in the packaging originally approved } \\
\text { by the Food and Drug Administration..." }\end{array}$ \\
\hline $\begin{array}{l}\text { Blinding of outcome assessment (detection } \\
\text { bias) } \\
\text { All outcomes }\end{array}$ & High risk & $\begin{array}{l}\text { Investigator was masked to treatment allocation (p. } \\
1733 \text { - material and methods): “...but the single in- } \\
\text { vestigator was masked as to which treatment the pa- } \\
\text { tient was currently using." }\end{array}$ \\
\hline
\end{tabular}

Melamed 1994

\begin{tabular}{ll}
\hline Methods & Parallel-group RCT \\
\hline Participants & 86 participants with seasonal allergic conjunctivitis \\
\hline Interventions & Two treatment arms: nedocromil sodium; placebo. Duration of treatment 8 weeks \\
\hline Outcomes & $\begin{array}{l}\text { Participants' assessment of symptoms -- sum score itching, burning, tearing, overall eye } \\
\text { condition } \\
\text { Clinicians' assessment of ocular signs and symptoms (using a scale } 0=\text { none, } 4=\text { very } \\
\text { severe) } \\
\text { Time points: at baseline and peak pollen period during the } 8 \text { weeks of treatment }\end{array}$
\end{tabular}

Topical antihistamines and mast cell stabilisers for treating seasonal and perennial allergic conjunctivitis (Review)

Copyright @ 2015 The Cochrane Collaboration. Published by John Wiley \& Sons, Ltd. 
Melamed 1994 (Continued)

\begin{tabular}{|c|c|c|}
\hline Country & \multicolumn{2}{|l|}{ United States } \\
\hline $\begin{array}{l}\text { Number randomised, gender (male:fe- } \\
\text { male) }\end{array}$ & \multicolumn{2}{|c|}{86 participants randomised, 85 participants analysed. M:F 49:36 } \\
\hline Age mean (SD), median, range & \multicolumn{2}{|c|}{ Mean (range) nedocromil sodium 33.1 years (13-60); placebo 31.1 years (13-57) } \\
\hline Notes & \multicolumn{2}{|c|}{$\begin{array}{l}\text { Not reported when study was conducted. Source of funding: support in part by Fisons } \\
\text { Pharmaceuticals. Declaration of interest by the authors was not stated }\end{array}$} \\
\hline \multicolumn{3}{|l|}{ Risk of bias } \\
\hline Bias & Authors' judgement & Support for judgement \\
\hline $\begin{array}{l}\text { Random sequence generation (selection } \\
\text { bias) }\end{array}$ & Unclear risk & $\begin{array}{l}\text { The method used to generate the allocation sequence was not } \\
\text { described }\end{array}$ \\
\hline Allocation concealment (selection bias) & Unclear risk & $\begin{array}{l}\text { The method used to conceal the allocation sequence was not } \\
\text { described }\end{array}$ \\
\hline $\begin{array}{l}\text { Incomplete outcome data (attrition bias) } \\
\text { All outcomes }\end{array}$ & Low risk & $\begin{array}{l}\text { Data were nearly fully reported, with less than } 10 \% \text { lost to fol- } \\
\text { low-up, and reasons were given }\end{array}$ \\
\hline Selective reporting (reporting bias) & Low risk & Evidence was available of proper outcome reporting \\
\hline Other bias & Low risk & No apparent evidence of other risk of bias \\
\hline $\begin{array}{l}\text { Blinding of participants and personnel } \\
\text { (performance bias) } \\
\text { All outcomes }\end{array}$ & Low risk & $\begin{array}{l}\text { Double-masked and identical package (p.58 - study design): } \\
\text { "The study was designed as a multicenter, randomized, double- } \\
\text { masked... Active and placebo drugs were packaged identically } \\
\text { and labeled in opaque polyethylene bottles..." }\end{array}$ \\
\hline $\begin{array}{l}\text { Blinding of outcome assessment (detection } \\
\text { bias) } \\
\text { All outcomes }\end{array}$ & Low risk & $\begin{array}{l}\text { Double-masked and identical package (p. } 58 \text { - study design): } \\
\text { "The study was designed as a multicenter, randomized, double- } \\
\text { masked... Active and placebo drugs were packaged identically } \\
\text { and labeled in opaque polyethylene bottles..." }\end{array}$ \\
\hline
\end{tabular}

\section{Melamed 2000}

\begin{tabular}{ll}
\hline Methods & Parallel-group RCT \\
\hline Participants & 189 participants with seasonal allergic conjunctivitis \\
\hline Interventions & Two treatment arms: nedocromil sodium 2\%; vehicle. Duration of treatment 8 weeks
\end{tabular}


Melamed 2000 (Continued)

\begin{tabular}{ll}
\hline Outcomes & $\begin{array}{l}\text { Participants' assessment of various symptoms (composite score itching, burning, tearing, } \\
\text { overall eye condition) and diary cards (using a scale } 0=\text { none, } 4=\text { very severe) to assess } \\
\text { individual symptoms at treatment } \\
\text { Clinicians' assessment of ocular signs and symptoms, and clinician and participant overall } \\
\text { opinions of treatment effectiveness at baseline and peak pollen season } \\
\text { Time scale: during the } 8 \text { weeks of treatment }\end{array}$ \\
\hline Country & United States \\
\hline
\end{tabular}

Number randomised, gender (male:fe- 189 participants randomised. M:F 104:85 male)

Age mean (SD), median, range Mean (range): nedocromil sodium 33.4 years (12-65); vehicle 31.0 years (13-67)

Notes

Study conducted in August 1986. Source of funding: in part by Fisons Pharmaceuticals. The authors did not have a financial interest in the drugs discussed within this manuscript

\section{Risk of bias}

\begin{tabular}{|c|c|c|}
\hline Bias & Authors' judgement & Support for judgement \\
\hline $\begin{array}{l}\text { Random sequence generation (selection } \\
\text { bias) }\end{array}$ & Unclear risk & $\begin{array}{l}\text { The method used to generate the allocation sequence was not } \\
\text { described }\end{array}$ \\
\hline Allocation concealment (selection bias) & Unclear risk & $\begin{array}{l}\text { The method used to conceal the allocation sequence was not } \\
\text { described }\end{array}$ \\
\hline $\begin{array}{l}\text { Incomplete outcome data (attrition bias) } \\
\text { All outcomes }\end{array}$ & Low risk & $\begin{array}{l}\text { Data were nearly fully reported, with less than } 10 \% \text { lost to fol- } \\
\text { low-up with similar percentage of participants lost to follow-up } \\
\text { in each arm, and reasons were given }\end{array}$ \\
\hline Selective reporting (reporting bias) & Low risk & Evidence was available of proper outcome reporting \\
\hline Other bias & Low risk & No apparent evidence of other risk of bias \\
\hline $\begin{array}{l}\text { Blinding of participants and personnel } \\
\text { (performance bias) } \\
\text { All outcomes }\end{array}$ & Low risk & $\begin{array}{l}\text { Double-masked (p. } 236 \text { - study design): "The nine week studies } \\
\text { used a double-blind, placebo-controlled..." } \\
\text { Identical bottles (p. } 236 \text { - study protocol): "At the end of the } \\
\text { baseline week, patients were randomised to receive either one } \\
\text { drop of nedocromil sodium } 2 \% \text { or vehicle solution b.i.d in each } \\
\text { eye, delivered from identical opaque bottles." }\end{array}$ \\
\hline $\begin{array}{l}\text { Blinding of outcome assessment (detection } \\
\text { bias) } \\
\text { All outcomes }\end{array}$ & Low risk & $\begin{array}{l}\text { Double-masked (p.236 - study design): "The nine week studies } \\
\text { used a double-blind, placebo-controlled..." } \\
\text { Identical bottles (p. } 236 \text { - study protocol): "At the end of the } \\
\text { baseline week, patients were randomised to receive either one } \\
\text { drop of nedocromil sodium } 2 \% \text { or vehicle solution b.i.d in each } \\
\text { eye, delivered from identical opaque bottles." }\end{array}$ \\
\hline
\end{tabular}


Möller 1994

\begin{tabular}{ll}
\hline Methods & Parallel-group RCT \\
\hline Participants & 149 participants with seasonal allergic conjunctivitis \\
\hline Interventions & Two treatment arms: nedocromil sodium; placebo. Duration of treatment 4 weeks \\
\hline Outcomes & $\begin{array}{l}\text { Investigator's and participants' (or their parents') assessment of severity of symptoms. } \\
\text { Mean score of itching, redness, soreness, grittiness, photophobia, and general eye con- } \\
\text { dition (using a 5-point scale; } 0 \text { = none, } 4 \text { = very severe) } \\
\text { Time points: during peak pollen in the } 4 \text { weeks of treatment }\end{array}$ \\
\hline Country & Sweden \\
\hline
\end{tabular}

Number randomised, gender (male:fe- 149 participants randomised, 146 participants analysed. M:F 92:57 male)

Age mean (SD), median, range Mean (range): nedocromil sodium 12 years (6-16); placebo 13 years (6-16)

Notes $\quad$ Not reported when study was conducted. Source of funding: Fisons Pharmaceuticals.

Declaration of interest by the authors was not stated

\section{Risk of bias}

Bias

Random sequence generation (selection Low risk bias)

Allocation concealment (selection bias) Low risk
Incomplete outcome data (attrition bias) Low risk All outcomes
Low risk

Low risk

Use of predetermined randomised code (p.885 - patients and methods): "Patients were then allocated, by a predetermined randomised code... Trial supplies were coded at source, by computer-generated numbers randomised in a balanced blocks of four"

Conceal allocation described (p.885 - patients and methods): "Patients entering the trial were assigned to treatment numbers in sequence. The codes were held in individual sealed envelopes by the investigator, to be returned unopened after the trial."

Data were nearly fully reported, with less than $10 \%$ lost to follow-up, and reasons were given

Selective reporting (reporting bias)

Evidence was available of proper outcome reporting

Other bias

Low risk

Blinding of participants and personnel Low risk (performance bias)

All outcomes

Double-masked (p.884 - summary): "This was a multicentre, double-blind...”

(p.885 - patients and methods): "active or placebo eye drops (both contained... in addition, the placebo eye drops contained 
$0.0005 \%$ rivoflavin as a yellow colorant to match the $2 \%$ nedocromil sodium content of the active eye drops).”

Blinding of outcome assessment (detection Low risk bias)

All outcomes
Double-masked (p.884 - summary): "This was a multicentre, double-blind...”

(p.885 - patients and methods): "active or placebo eye drops (both contained... in addition, the placebo eye drops contained $0.0005 \%$ rivoflavin as a yellow colorant to match the $2 \%$ nedocromil sodium content of the active eye drops)."

\begin{tabular}{|c|c|c|}
\hline Methods & \multicolumn{2}{|l|}{ Parallel-group RCT } \\
\hline Participants & \multicolumn{2}{|c|}{116 participants with moderate to severe perennial allergic conjunctivitis } \\
\hline Interventions & \multicolumn{2}{|c|}{ Two treatment arms: azelastine $0.015 \mathrm{mg}$; placebo. Duration of treatment 6 weeks } \\
\hline Outcomes & \multicolumn{2}{|c|}{$\begin{array}{l}\text { Participants' and clinicians' assessment of ocular symptoms (sum score itching and red- } \\
\text { ness, using a 4-point scale; } 0=\text { none, } 3=\text { severe) } \\
\text { Time points: at baseline and day } 7,21 \text {, and } 42 \text { of treatment }\end{array}$} \\
\hline Country & \multicolumn{2}{|l|}{ Germany } \\
\hline $\begin{array}{l}\text { Number randomised, gender (male:fe- } \\
\text { male) }\end{array}$ & \multicolumn{2}{|c|}{116 participants randomised and analysed. M:F $26: 90$} \\
\hline Age mean (SD), median, range & \multicolumn{2}{|c|}{ Mean (range): azelastine 35.7 years (17-63); placebo 31.7 years $(17-59)$} \\
\hline Notes & \multicolumn{2}{|c|}{$\begin{array}{l}\text { Study conducted from } 19 \text { December } 1998 \text { to } 14 \text { April } 1999 \text {. Source of funding not } \\
\text { stated. Declaration of interest by the authors was not stated }\end{array}$} \\
\hline \multicolumn{3}{|l|}{ Risk of bias } \\
\hline Bias & Authors' judgement & Support for judgement \\
\hline $\begin{array}{l}\text { Random sequence generation (selection } \\
\text { bias) }\end{array}$ & Unclear risk & $\begin{array}{l}\text { The method used to generate the allocation sequence was not } \\
\text { described }\end{array}$ \\
\hline Allocation concealment (selection bias) & Unclear risk & $\begin{array}{l}\text { The method used to conceal the allocation sequence was not } \\
\text { described }\end{array}$ \\
\hline $\begin{array}{l}\text { Incomplete outcome data (attrition bias) } \\
\text { All outcomes }\end{array}$ & Low risk & $\begin{array}{l}\text { Data were nearly fully reported, with less than } 10 \% \text { lost to fol- } \\
\text { low-up, and reasons were given }\end{array}$ \\
\hline Selective reporting (reporting bias) & Low risk & Evidence was available of proper outcome reporting \\
\hline
\end{tabular}

Topical antihistamines and mast cell stabilisers for treating seasonal and perennial allergic conjunctivitis (Review) 
Nazarov 2003 (Continued)

\begin{tabular}{|c|c|c|}
\hline Other bias & Low risk & No apparent evidence of other risk of bias \\
\hline $\begin{array}{l}\text { Blinding of participants and personnel } \\
\text { (performance bias) } \\
\text { All outcomes }\end{array}$ & Low risk & $\begin{array}{l}\text { Double-masked (p.169 - patients, materials and methods): "The } \\
\text { investigation was conducted as a double-blind, randomised..." } \\
\text { Both drugs with identical packaging (p.169 - treatment): "Both } \\
\text { azelastine eye drops and the matching vehicle containing placebo } \\
\text { were provided by ASTA Medica AG in identical packaging." }\end{array}$ \\
\hline $\begin{array}{l}\text { Blinding of outcome assessment (detection } \\
\text { bias) } \\
\text { All outcomes }\end{array}$ & Low risk & $\begin{array}{l}\text { Double-masked (p.169 - patients, materials and methods): "The } \\
\text { investigation was conducted as a double-blind, randomised..." } \\
\text { Both drugs with identical packaging (p.169 - treatment): "Both } \\
\text { azelastine eye drops and the matching vehicle containing placebo } \\
\text { were provided by ASTA Medica AG in identical packaging." }\end{array}$ \\
\hline
\end{tabular}

Petzold 2002

\begin{tabular}{|c|c|c|}
\hline Methods & \multicolumn{2}{|c|}{ Parallel-group RCTs (3 RCTs reported together in abstract) } \\
\hline Participants & \multicolumn{2}{|c|}{$\begin{array}{l}\text { Study 3034: } 78 \text { participants; Study 3062: } 145 \text { participants; Study 3021: } 240 \text { participants } \\
\text { with allergic conjunctivitis }\end{array}$} \\
\hline Interventions & \multicolumn{2}{|c|}{ Two treatment arms: azelastine $0.05 \%$; placebo. Duration of treatment 14 or 28 days } \\
\hline Outcomes & \multicolumn{2}{|c|}{$\begin{array}{l}\text { Investigators' assessment of ocular symptoms (itching and redness) using a 4-point scale } \\
(0=\text { none, } 3=\text { severe }) \\
\text { Time scale: day } 0,3 \text {, and } 14 \text { of treatment }\end{array}$} \\
\hline Country & \multicolumn{2}{|l|}{ Europe } \\
\hline $\begin{array}{l}\text { Number randomised, gender (male:fe- } \\
\text { male) }\end{array}$ & \multicolumn{2}{|c|}{$\begin{array}{l}\text { Study 3034: } 78 \text { participants (number of participants in each arm uncertain) randomised } \\
\text { Study 3062: } 145 \text { participants (number of participants in each arm uncertain) } \\
\text { Study 3021: } 240 \text { participants (number of participants in each arm uncertain). Gender } \\
\text { not stated (abstract) }\end{array}$} \\
\hline Age mean (SD), median, range & \multicolumn{2}{|l|}{ Age range $4-12$ years } \\
\hline Notes & \multicolumn{2}{|c|}{$\begin{array}{l}\text { Not reported when study was conducted. Source of funding not stated. Declaration of } \\
\text { interest by the authors was not stated }\end{array}$} \\
\hline \multicolumn{3}{|l|}{ Risk of bias } \\
\hline Bias & Authors' judgement & Support for judgement \\
\hline $\begin{array}{l}\text { Random sequence generation (selection } \\
\text { bias) }\end{array}$ & Unclear risk & Only abstract available \\
\hline Allocation concealment (selection bias) & Unclear risk & Only abstract available \\
\hline
\end{tabular}


Petzold 2002 (Continued)

\begin{tabular}{l|ll}
\hline $\begin{array}{l}\text { Incomplete outcome data (attrition bias) } \\
\text { All outcomes }\end{array}$ & Unclear risk & Only abstract available \\
\hline Selective reporting (reporting bias) & Unclear risk & Only abstract available \\
\hline Other bias & Unclear risk & Only abstract available \\
\hline $\begin{array}{l}\text { Blinding of participants and personnel } \\
\text { (performance bias) } \\
\text { All outcomes }\end{array}$ & Unclear risk & Only abstract available \\
\hline $\begin{array}{l}\text { Blinding of outcome assessment (detection } \\
\text { bias) } \\
\text { All outcomes }\end{array}$ & Unclear risk & Only abstract available \\
\hline
\end{tabular}

\section{Sabbah 1998}

\begin{tabular}{ll}
\hline Methods & Parallel-group RCT \\
\hline Participants & 113 participants with seasonal allergic conjunctivitis/rhinoconjunctivitis \\
\hline Interventions & Three treatment arms: azelastine; levocabastine; placebo. Duration of treatment 14 days \\
\hline Outcomes & $\begin{array}{l}\text { Participants' (using diaries) and physicians' assessments (sum scores) based on a decrease } \\
\text { of the average score }>3 \text { units for three main eye symptoms (ocular itching, tearing, and } \\
\text { redness) } \\
\text { Time points: at baseline and day } 3 \text { and } 14 \text { of treatment }\end{array}$ \\
\hline Country & France \\
\hline
\end{tabular}

Number randomised, gender (male:fe- 113 participants randomised, 107 participants analysed. M:F 75:38 male)

Age mean (SD), median, range

Notes
Mean (SD): azelastine 8.3 years (2.4); levocabastine 8.2 years (2.5); placebo 8.3 years (2. 3)

Not reported when study was conducted. Source of funding Laboratoires ASTA Medica. Declaration of interest by the authors was not stated

\section{Risk of bias}

\section{Bias}

Random sequence generation (selection Unclear risk bias)

\section{Authors' judgement Support for judgement}

The method used to generate the allocation sequence was not described 


\section{Sabbah 1998 (Continued)}

\begin{tabular}{|c|c|c|}
\hline Allocation concealment (selection bias) & Unclear risk & $\begin{array}{l}\text { The method used to conceal the allocation sequence was not } \\
\text { described }\end{array}$ \\
\hline $\begin{array}{l}\text { Incomplete outcome data (attrition bias) } \\
\text { All outcomes }\end{array}$ & Low risk & $\begin{array}{l}10 \% \text { attrition, with similar numbers lost to follow-up between } \\
\text { arms }\end{array}$ \\
\hline Selective reporting (reporting bias) & Low risk & Primary outcome result was reported according to protocol \\
\hline Other bias & Low risk & No evidence of other risk of bias \\
\hline $\begin{array}{l}\text { Blinding of participants and personnel } \\
\text { (performance bias) } \\
\text { All outcomes }\end{array}$ & Unclear risk & $\begin{array}{l}\text { The study did not describe details on masking of participants } \\
\text { on the intervention allocations. Masking was not done with } \\
\text { levocabastine due to labelling, but double-masked with other } \\
\text { interventions }\end{array}$ \\
\hline $\begin{array}{l}\text { Blinding of outcome assessment (detection } \\
\text { bias) } \\
\text { All outcomes }\end{array}$ & Unclear risk & $\begin{array}{l}\text { The study did not describe details on masking of personnel/ } \\
\text { investigators on the intervention allocations. Masking was not } \\
\text { done with levocabastine due to labelling, but double-masked } \\
\text { with other interventions }\end{array}$ \\
\hline
\end{tabular}

Sarker 2011

\begin{tabular}{|c|c|}
\hline Methods & Parallel-group RCT \\
\hline Participants & 92 participants with allergic conjunctivitis \\
\hline Interventions & $\begin{array}{l}\text { Two treatment arms: ketotifen fumarate } 0.025 \% \text {; olopatadine hydrochloride } 0.1 \% \text {. Du- } \\
\text { ration of treatment } 2 \text { weeks }\end{array}$ \\
\hline Outcomes & $\begin{array}{l}\text { Assessment of ocular sign and symptoms (mean scores) for itching, tearing, hyperaemia, } \\
\text { photophobia (using a 4-point scale; } 0=\text { none, } 3=\text { severe) } \\
\text { Time points: at baseline and } 2 \text { weeks treatment period } \\
\text { Unclear if outcomes were measured by participants or clinicians, or both }\end{array}$ \\
\hline Country & Bangladesh \\
\hline $\begin{array}{l}\text { Number randomised, gender (male:fe- } \\
\text { male) }\end{array}$ & 92 participants randomised, 83 participants analysed. M:F 36:47 \\
\hline Age mean (SD), median, range & Mean (SD): ketotifen 28 years (12); olopatadine 28 years (11). Overall range $12-50$ years \\
\hline Notes & $\begin{array}{l}\text { Source of funding not stated. The authors have no relevant affiliations or financial } \\
\text { involvement with any organization or entity with a financial interest in or financial } \\
\text { conflict with the subject matter or materials discussed in the manuscript }\end{array}$ \\
\hline
\end{tabular}

\section{Risk of bias}

Bias

Authors' judgement Support for judgement

Topical antihistamines and mast cell stabilisers for treating seasonal and perennial allergic conjunctivitis (Review)

Copyright @ 2015 The Cochrane Collaboration. Published by John Wiley \& Sons, Ltd. 


\section{Sarker 2011 (Continued)}

\begin{tabular}{|c|c|c|}
\hline $\begin{array}{l}\text { Random sequence generation (selection } \\
\text { bias) }\end{array}$ & Low risk & $\begin{array}{l}\text { Randomisation list (p. } 547 \text { - treatment allocation and follow-up) } \\
\text { :Patients who were found to be eligible according to selection } \\
\text { criteria were recruited in to one of the treatment groups accord- } \\
\text { ing to a stratified randomisation list based on age and sex." }\end{array}$ \\
\hline Allocation concealment (selection bias) & Unclear risk & $\begin{array}{l}\text { The method used to conceal the allocation sequence was not } \\
\text { described }\end{array}$ \\
\hline $\begin{array}{l}\text { Incomplete outcome data (attrition bias) } \\
\text { All outcomes }\end{array}$ & Low risk & $\begin{array}{l}\text { Data were nearly fully reported, with less than } 10 \% \text { lost to fol- } \\
\text { low-up, and reasons were given }\end{array}$ \\
\hline Selective reporting (reporting bias) & Low risk & Evidence was available of proper outcome reporting \\
\hline Other bias & Low risk & No apparent evidence of other risk of bias \\
\hline $\begin{array}{l}\text { Blinding of participants and personnel } \\
\text { (performance bias) } \\
\text { All outcomes }\end{array}$ & Low risk & $\begin{array}{l}\text { Double-masked (p.547 - treatment allocation and follow-up): } \\
\text { "Study medications were provided in identical containers so that } \\
\text { both patients and investigators remained blinded." }\end{array}$ \\
\hline $\begin{array}{l}\text { Blinding of outcome assessment (detection } \\
\text { bias) } \\
\text { All outcomes }\end{array}$ & Low risk & $\begin{array}{l}\text { Study conducted from } 1 \text { January to } 31 \text { December } 2007 \text {. Double- } \\
\text { masked (p. } 547 \text { - treatment allocation and follow-up): "Study } \\
\text { medications were provided in identical containers so that both } \\
\text { patients and investigators remained blinded." }\end{array}$ \\
\hline
\end{tabular}

\section{Secchi 2000a}

\begin{tabular}{|c|c|}
\hline Methods & Parallel-group RCT \\
\hline Participants & 42 paediatric participants with allergic conjunctivitis \\
\hline Interventions & $\begin{array}{l}\text { Two treatment arms: emedastine } 0.05 \% \text {; levocabastine } 0.05 \% \text {. Duration of treatment } \\
42 \text { days }\end{array}$ \\
\hline Outcomes & $\begin{array}{l}\text { Investigator assessment of ocular signs (using a scale } 0-4 \text { ) at days } 3,7,14,30,42 \text {, and } \\
\text { overall progress } \\
\text { Participant assessment of ocular symptoms itching and redness (using a visual analogue } \\
\text { scale; } 0=\text { none, } 9=\text { severe) } \\
\text { Time points: at days } 7,14,30,42 \text { of treatment }\end{array}$ \\
\hline Country & Italy \\
\hline $\begin{array}{l}\text { Number randomised, gender (male:fe- } \\
\text { male) }\end{array}$ & 42 participants randomised, 38 participants analysed. Gender not stated \\
\hline
\end{tabular}

Age mean (SD), median, range

Age range 4-6 years: 11 participants

Age range $7-16$ years: 31 participants 


\section{Secchi 2000a (Continued)}

$\begin{array}{ll}\text { Notes } & \begin{array}{l}\text { Not reported when study was conducted. Source of funding not stated. Declaration of } \\ \text { interest by the authors was not stated }\end{array}\end{array}$

\section{Risk of bias}

Bias

Authors' judgement Support for judgement

\begin{tabular}{|c|c|c|}
\hline $\begin{array}{l}\text { Random sequence generation (selection } \\
\text { bias) }\end{array}$ & Unclear risk & $\begin{array}{l}\text { The method used to generate the allocation sequence was not } \\
\text { described }\end{array}$ \\
\hline Allocation concealment (selection bias) & Unclear risk & $\begin{array}{l}\text { The method used to conceal the allocation sequence was not } \\
\text { described }\end{array}$ \\
\hline $\begin{array}{l}\text { Incomplete outcome data (attrition bias) } \\
\text { All outcomes }\end{array}$ & Low risk & $\begin{array}{l}\text { Data were nearly fully reported, with } 10 \% \text { lost to follow-up, } \\
\text { reasons were given for one case only }\end{array}$ \\
\hline Selective reporting (reporting bias) & Low risk & Evidence was available of proper outcome reporting \\
\hline Other bias & Low risk & No apparent evidence of other risk of bias \\
\hline $\begin{array}{l}\text { Blinding of participants and personnel } \\
\text { (performance bias) } \\
\text { All outcomes }\end{array}$ & Low risk & $\begin{array}{l}\text { Double-masked (p. } 42 \text { - abstract, material and methods): "In a } \\
\text { randomised, double-masked... Subjects who met all inclusion } \\
\text { and exclusion criteria received masked study medication with } \\
\text { instructions to instill drops..." }\end{array}$ \\
\hline
\end{tabular}

Blinding of outcome assessment (detection Low risk bias)

Double-masked (p.42 - abstract, material and methods): "In a

randomised, double-masked..."

All outcomes

\section{Secchi 2000b}

\begin{tabular}{ll}
\hline Methods & Parallel-group RCT \\
\hline Participants & 222 participants (including 42 paediatric) with seasonal allergic conjunctivitis \\
\hline Interventions & $\begin{array}{l}\text { Two treatment arms: emedastine } 0.05 \% \text {; levocabastine } 0.05 \% . \text { Duration of treatment } \\
42 \text { days }\end{array}$ \\
\hline Outcomes & $\begin{array}{l}\text { Investigator assessment of ocular signs (using a scale } 0-4) \text { at days } 3,7,14,30,42 \text { Partici- } \\
\text { pant assessment of ocular symptoms (using a visual analogue scale; } 0=\text { none, } 9=\text { severe) } \\
\text { at days } 7,14,30,42\end{array}$ \\
\hline Country & Italy and United States \\
\hline
\end{tabular}

Number randomised, gender (male:fe- 202 participants randomised. M:F 107:114 male) 


\section{Secchi 2000b (Continued)}

\begin{tabular}{|c|c|c|}
\hline Age mean $(\mathrm{SD})$, median, range & \multicolumn{2}{|c|}{ Average age (range): 30 years (4-76) } \\
\hline Notes & \multicolumn{2}{|c|}{$\begin{array}{l}\text { Not reported when study was conducted. Source of funding not stated. Declaration of } \\
\text { interest by the authors was not stated }\end{array}$} \\
\hline \multicolumn{3}{|l|}{ Risk of bias } \\
\hline Bias & Authors' judgement & Support for judgement \\
\hline $\begin{array}{l}\text { Random sequence generation (selection } \\
\text { bias) }\end{array}$ & Unclear risk & $\begin{array}{l}\text { The method used to generate the allocation sequence was not } \\
\text { described }\end{array}$ \\
\hline Allocation concealment (selection bias) & Unclear risk & $\begin{array}{l}\text { The method used to conceal the allocation sequence was not } \\
\text { described }\end{array}$ \\
\hline $\begin{array}{l}\text { Incomplete outcome data (attrition bias) } \\
\text { All outcomes }\end{array}$ & Low risk & $\begin{array}{l}\text { Data were nearly fully reported for each intervention group, with } \\
\text { less than } 20 \% \text { lost to follow-up (Figure } 1 \text { of the trial report). A } \\
\text { similar percentage of participants was lost to follow-up in each } \\
\text { arm }\end{array}$ \\
\hline Selective reporting (reporting bias) & Unclear risk & Evidence was available of proper outcome reporting \\
\hline Other bias & Low risk & No apparent evidence of other risk of bias \\
\hline $\begin{array}{l}\text { Blinding of participants and personnel } \\
\text { (performance bias) } \\
\text { All outcomes }\end{array}$ & Low risk & $\begin{array}{l}\text { Double-masked (p. } 49 \text { - material and methods): "In a random- } \\
\text { ized, double-masked..." }\end{array}$ \\
\hline $\begin{array}{l}\text { Blinding of outcome assessment (detection } \\
\text { bias) } \\
\text { All outcomes }\end{array}$ & Low risk & $\begin{array}{l}\text { Double-masked (p. } 49 \text { - material and methods): "In a random- } \\
\text { ized, double-masked..." }\end{array}$ \\
\hline
\end{tabular}

Trinquand 1999

\begin{tabular}{l|l}
\hline Methods & Parallel-group RCT \\
\hline Participants & 357 participants with perennial or seasonal allergic conjunctivitis \\
\hline Interventions & Two treatment arms: mequitazine; levocabastine. Duration of treatment 4 weeks \\
\hline Outcomes & $\begin{array}{l}\text { Investigators' assessment of symptoms at day 7, 14, and 28 Participants' assessment of } \\
\text { symptoms (diary cards) }\end{array}$ \\
\hline Country & Not stated \\
\hline
\end{tabular}

Number randomised, gender (male:fe- 357 participants randomised male) 
Trinquand 1999 (Continued)

\begin{tabular}{|c|c|c|}
\hline Age mean (SD), median, range & \multicolumn{2}{|l|}{ Not stated } \\
\hline Notes & \multicolumn{2}{|c|}{$\begin{array}{l}\text { Not reported when study was conducted. Full-text paper not available, only abstract } \\
\text { therefore insufficient information to assess risk of bias. Source of funding and declaration } \\
\text { of interest by the authors were not stated }\end{array}$} \\
\hline \multicolumn{3}{|l|}{ Risk of bias } \\
\hline Bias & Authors' judgement & Support for judgement \\
\hline $\begin{array}{l}\text { Random sequence generation (selection } \\
\text { bias) }\end{array}$ & Unclear risk & Only abstract available \\
\hline Allocation concealment (selection bias) & Unclear risk & Only abstract available \\
\hline $\begin{array}{l}\text { Incomplete outcome data (attrition bias) } \\
\text { All outcomes }\end{array}$ & Unclear risk & Only abstract available \\
\hline Selective reporting (reporting bias) & Unclear risk & Only abstract available \\
\hline Other bias & Unclear risk & Only abstract available \\
\hline $\begin{array}{l}\text { Blinding of participants and personnel } \\
\text { (performance bias) } \\
\text { All outcomes }\end{array}$ & Unclear risk & Only abstract available \\
\hline $\begin{array}{l}\text { Blinding of outcome assessment (detection } \\
\text { bias) } \\
\text { All outcomes }\end{array}$ & Unclear risk & Only abstract available \\
\hline
\end{tabular}

\section{Varguez-Rodriguez 2009}

\begin{tabular}{|c|c|}
\hline Methods & Parallel-group RCT \\
\hline Participants & 40 participants (naive-treatment) with seasonal allergic conjunctivitis \\
\hline Interventions & $\begin{array}{l}\text { Two treatment arms: olopatadine } 0.1 \% \text {; ketotifen } 0.025 \% \text {. Duration of treatment } 4 \\
\text { weeks }\end{array}$ \\
\hline Outcomes & $\begin{array}{l}\text { Assessment of ocular symptoms: itching, watering, burning (range } 0-3 ; 0=\text { none, } 3= \\
\text { severe) and signs: redness, chemosis (absent, present) } \\
\text { Unclear if outcomes were measured by participants or clinicians, or both }\end{array}$ \\
\hline Country & Mexico \\
\hline
\end{tabular}

Number randomised, gender (male:fe- 40 participants randomised. M:F 23:17 male) 


\begin{tabular}{|c|c|c|}
\hline Age mean (SD), median, range & \multicolumn{2}{|c|}{ Mean (SD): olopatadine 19.7 years (6.6); ketotifen 21.05 years $(8.3)$} \\
\hline Notes & \multicolumn{2}{|c|}{ Source of funding not stated. Declaration of interest by the authors was not stated } \\
\hline \multicolumn{3}{|l|}{ Risk of bias } \\
\hline Bias & Authors' judgement & Support for judgement \\
\hline $\begin{array}{l}\text { Random sequence generation (selection } \\
\text { bias) }\end{array}$ & Unclear risk & $\begin{array}{l}\text { The method used to generate the allocation sequence was not } \\
\text { described }\end{array}$ \\
\hline Allocation concealment (selection bias) & Unclear risk & $\begin{array}{l}\text { The method used to conceal the allocation sequence was not } \\
\text { described }\end{array}$ \\
\hline $\begin{array}{l}\text { Incomplete outcome data (attrition bias) } \\
\text { All outcomes }\end{array}$ & Low risk & $\begin{array}{l}\text { Complete outcome data reported, all the participants ran- } \\
\text { domised were analysed }\end{array}$ \\
\hline Selective reporting (reporting bias) & Low risk & Evidence was available of proper outcome reporting \\
\hline Other bias & Low risk & No apparent evidence of other risk of bias \\
\hline $\begin{array}{l}\text { Blinding of participants and personnel } \\
\text { (performance bias) } \\
\text { All outcomes }\end{array}$ & Low risk & $\begin{array}{l}\text { Masking of participants (p. } 401 \text { - methods): "All the bottles had } \\
\text { the same appearance and the patient did not know the treatment } \\
\text { received." }\end{array}$ \\
\hline $\begin{array}{l}\text { Blinding of outcome assessment (detection } \\
\text { bias) } \\
\text { All outcomes }\end{array}$ & Low risk & $\begin{array}{l}\text { Masking of investigators (p. } 401 \text { - methods): "All the bottles had } \\
\text { the same appearance and the patient did not know the treatment } \\
\text { received." }\end{array}$ \\
\hline
\end{tabular}

\section{Verin 2001}

\begin{tabular}{ll}
\hline Methods & Parallel-group RCT \\
\hline Participants & 222 participants with seasonal allergic conjunctivitis \\
\hline Interventions & Two treatment arms: levocabastine; emedastine. Duration of treatment 6 weeks \\
\hline Outcomes & $\begin{array}{l}\text { Participants assessment of itching and redness using diaries and visual analogue scale (0 } \\
\text { = none; } 9=\text { severe }) \\
\text { Physician assessment of itching, redness } \\
\text { Time points: day } 0-14 \text { and day } 30-42 \text { of treatment }\end{array}$ \\
\hline Country & Europe, South Africa, and Australia \\
\hline
\end{tabular}

Number randomised, gender (male:fe- 222 participants randomised, 202 participants analysed. Gender not stated male) 
Verin 2001 (Continued)

\begin{tabular}{|c|c|c|}
\hline Age mean (SD), median, range & \multicolumn{2}{|c|}{ Mean (range) 30 years (4-76) } \\
\hline Notes & \multicolumn{2}{|c|}{$\begin{array}{l}\text { Not reported when study was conducted. Source of funding Alcon Research Ltd. Dec- } \\
\text { laration of interest by the authors was not stated }\end{array}$} \\
\hline \multicolumn{3}{|l|}{ Risk of bias } \\
\hline Bias & Authors' judgement & Support for judgement \\
\hline $\begin{array}{l}\text { Random sequence generation (selection } \\
\text { bias) }\end{array}$ & Unclear risk & $\begin{array}{l}\text { The method used to generate the allocation sequence was not } \\
\text { described }\end{array}$ \\
\hline Allocation concealment (selection bias) & Unclear risk & $\begin{array}{l}\text { The method used to conceal the allocation sequence was not } \\
\text { described }\end{array}$ \\
\hline $\begin{array}{l}\text { Incomplete outcome data (attrition bias) } \\
\text { All outcomes }\end{array}$ & Unclear risk & Not intention-to-treat analysis \\
\hline Selective reporting (reporting bias) & Low risk & Evidence was available of proper outcome reporting \\
\hline Other bias & Low risk & No apparent evidence of other risk of bias \\
\hline $\begin{array}{l}\text { Blinding of participants and personnel } \\
\text { (performance bias) } \\
\text { All outcomes }\end{array}$ & Low risk & $\begin{array}{l}\text { Identical containers (p. } 692 \text { - methods): "Study medications were } \\
\text { provided in identical containers so that both patients and inves- } \\
\text { tigators remained masked." }\end{array}$ \\
\hline $\begin{array}{l}\text { Blinding of outcome assessment (detection } \\
\text { bias) } \\
\text { All outcomes }\end{array}$ & Low risk & $\begin{array}{l}\text { Identical containers (p. } 692 \text { - methods): "Study medications were } \\
\text { provided in identical containers so that both patients and inves- } \\
\text { tigators remained masked." }\end{array}$ \\
\hline
\end{tabular}

Wertheimer 1997

\begin{tabular}{ll}
\hline Methods & Parallel-group RCT \\
\hline Participants & 69 participants with acute allergic conjunctivitis \\
\hline Interventions & $\begin{array}{l}\text { Two treatment arms: antazoline } 0.05 \% \text { and tetryzoline } 0.04 \% \text {; levocabastine } 0.05 \% . \\
\text { Duration of treatment } 2 \text { weeks }\end{array}$ \\
\hline Outcomes & $\begin{array}{l}\text { The total symptom score (Figure } 1 \text { of the trial report) of eight symptoms (each scored } \\
0=\text { none; } 3=\text { worst symptom). Four subjective and four objective symptoms; unclear } \\
\text { which symptoms were assessed by participants or clinicians }\end{array}$ \\
\hline Country & Germany \\
\hline
\end{tabular}

Number randomised, gender (male:fe- 69 participants randomised. M:F 35:34 male) 
Wertheimer 1997 (Continued)

\begin{tabular}{|c|c|c|}
\hline Age mean $(\mathrm{SD})$, median, range & \multicolumn{2}{|c|}{ Mean (SD): antazoline and tetryzoline 42.4 (15.4); levocabastine 43.1 (14.9) } \\
\hline Notes & \multicolumn{2}{|c|}{$\begin{array}{l}\text { Study conducted from March to August } 1995 \text {. Source of funding not stated. Declaration } \\
\text { of interest by the authors was not stated }\end{array}$} \\
\hline \multicolumn{3}{|l|}{ Risk of bias } \\
\hline Bias & Authors' judgement & Support for judgement \\
\hline $\begin{array}{l}\text { Random sequence generation (selection } \\
\text { bias) }\end{array}$ & Low risk & $\begin{array}{l}\text { Random sequence generation (p.94): "The randomisation } \\
\text { schedule, created at random in blocks of four, assigned patients } \\
\text { to the medications and application instructions listed in Table } \\
2 \text { " }\end{array}$ \\
\hline Allocation concealment (selection bias) & Unclear risk & $\begin{array}{l}\text { The method used to conceal the allocation sequence was not } \\
\text { described }\end{array}$ \\
\hline $\begin{array}{l}\text { Incomplete outcome data (attrition bias) } \\
\text { All outcomes }\end{array}$ & Unclear risk & No dropouts were mentioned \\
\hline Selective reporting (reporting bias) & High risk & $\begin{array}{l}\text { Only P values that were statistically significant were reported (p. } \\
95 \text {, paragraph } 3 \text { ). No results were reported for outcomes that } \\
\text { were not statistically significant (p.94, paragraph } 8 \text { ). Numerical } \\
\text { results were not reported in the text for any outcomes except for } \\
\text { the numbers with blurred vision (p.95, paragraph } 4 \text { ) }\end{array}$ \\
\hline Other bias & Low risk & No apparent evidence of other risk of bias \\
\hline $\begin{array}{l}\text { Blinding of participants and personnel } \\
\text { (performance bias) } \\
\text { All outcomes }\end{array}$ & Unclear risk & $\begin{array}{l}\text { (p. 94, paragraph 3): "After } 30 \text { minutes the eyes were studied } \\
\text { again, without the doctor knowing which drops were adminis- } \\
\text { tered. The medication and the double-masked application in- } \\
\text { structions, which prevented early identification of the medica- } \\
\text { tion, were handed over to the patient at the end of the visit" }\end{array}$ \\
\hline $\begin{array}{l}\text { Blinding of outcome assessment (detection } \\
\text { bias) } \\
\text { All outcomes }\end{array}$ & Unclear risk & $\begin{array}{l}\text { Masked at } 30 \text {-minute assessment, later assessments were not } \\
\text { masked to participants, as treatments had different dose frequen- } \\
\text { cies ( } 2 \text { per day versus } 4 \text { per day). Assessment masked to clinicians } \\
\text { unclear }\end{array}$ \\
\hline
\end{tabular}

RCT: randomised controlled trial

SD: standard deviation 
Characteristics of excluded studies [ordered by study ID]

\begin{tabular}{|c|c|}
\hline Study & Reason for exclusion \\
\hline Abelson 2003 & Using conjunctival allergen challenge test \\
\hline Abelson 2004 & Using conjunctival allergen challenge test \\
\hline Artal 2000 & Unit of randomisation: eye (participants received different treatments on each eye) \\
\hline Borazan 2009 & Unit of randomisation: eye (participants received different treatments on each eye) \\
\hline Garay 2001 & Non-randomised controlled trial \\
\hline Higuchi 1979 & Non-randomised controlled trial \\
\hline Kamis 2006 & Unit of randomisation: eye (participants received different treatments on each eye) \\
\hline Leino 1994 & Comparing different concentrations and dosage of the same active drug \\
\hline Leonardi 2004 & Non-randomised controlled trial \\
\hline Longo 1979 & Non-randomised controlled trial \\
\hline Merayo 2003 & Non-randomised controlled trial \\
\hline Möller 1990 & Non-randomised controlled trial \\
\hline Napoli 2005 & Non-randomised controlled trial \\
\hline Pinto 2001 & Cost-effectiveness study (original study Verin 2001) \\
\hline Scadding 1999 & Non-randomised controlled trial \\
\hline Scoper 2007 & Non-randomised controlled trial \\
\hline Torkildsen 2008 & Unit of randomisation: eye (participants received different treatments in each eye) \\
\hline
\end{tabular}

Characteristics of studies awaiting assessment [ordered by study ID]

\section{Dharmistha 2013}

\begin{tabular}{ll}
\hline Methods & No details, unclear if it is a RCT \\
\hline Participants & Allergic conjunctivitis \\
\hline Interventions & Topical olopatadine hydrochloride $0.1 \%$ versus ketotifen fumarate $0.025 \%$ \\
\hline $\begin{array}{l}\text { Topical antihistamines and mast cell stabilisers for treating seasonal and perennial allergic conjunctivitis (Review) } \\
\text { Copyright } \odot 2015\end{array}$
\end{tabular}




\section{Dharmistha 2013 (Continued)}

\begin{tabular}{ll}
\hline Outcomes & Unclear \\
\hline Notes & None \\
\hline
\end{tabular}

\section{Jia 2012}

\begin{tabular}{ll}
\hline Methods & No details, unclear if it is a RCT \\
\hline Participants & Children with allergic conjunctivitis \\
\hline Interventions & Azelastine \\
\hline Outcomes & No details \\
\hline Notes & In Chinese language \\
\hline
\end{tabular}

Scandashree 2013

\begin{tabular}{ll}
\hline Methods & RCT \\
\hline Participants & Allergic conjunctivitis \\
\hline Interventions & $\begin{array}{l}\text { Olopatadine hydrochloride } 0.2 \% \text { ophthalmic solution once daily versus sodium cromoglycate } 2 \% \text { ophthalmic solu- } \\
\text { tion } 4 \text { times a day }\end{array}$ \\
\hline Outcomes & Efficacy and tolerability. No further details \\
\hline Notes & Reported at the 46th Annual Conference of the Indian Pharmacological Society, IPSCON Bangalore India, 2013 \\
\hline
\end{tabular}

RCT: randomised controlled trial 
DATA AND ANALYSES

Comparison 1. Olopatadine versus ketotifen

\begin{tabular}{lcccc} 
Outcome or subgroup title & $\begin{array}{c}\text { No. of } \\
\text { studies }\end{array}$ & $\begin{array}{c}\text { No. of } \\
\text { participants }\end{array}$ & Statistical method & Effect size \\
\hline 1 Itching at 14 days & 4 & 182 & Mean Difference (IV, Random, 95\% CI) & $-0.32[-0.59,-0.06]$ \\
2 Tearing at 14 days & 3 & 142 & Mean Difference (IV, Random, 95\% CI) & $-0.06[-0.35,0.22]$ \\
\hline
\end{tabular}

\section{Analysis I.I. Comparison I Olopatadine versus ketotifen, Outcome I Itching at I4 days.}

Review: Topical antihistamines and mast cell stabilisers for treating seasonal and perennial allergic conjunctivitis

Comparison: I Olopatadine versus ketotifen

Outcome: I Itching at 14 days

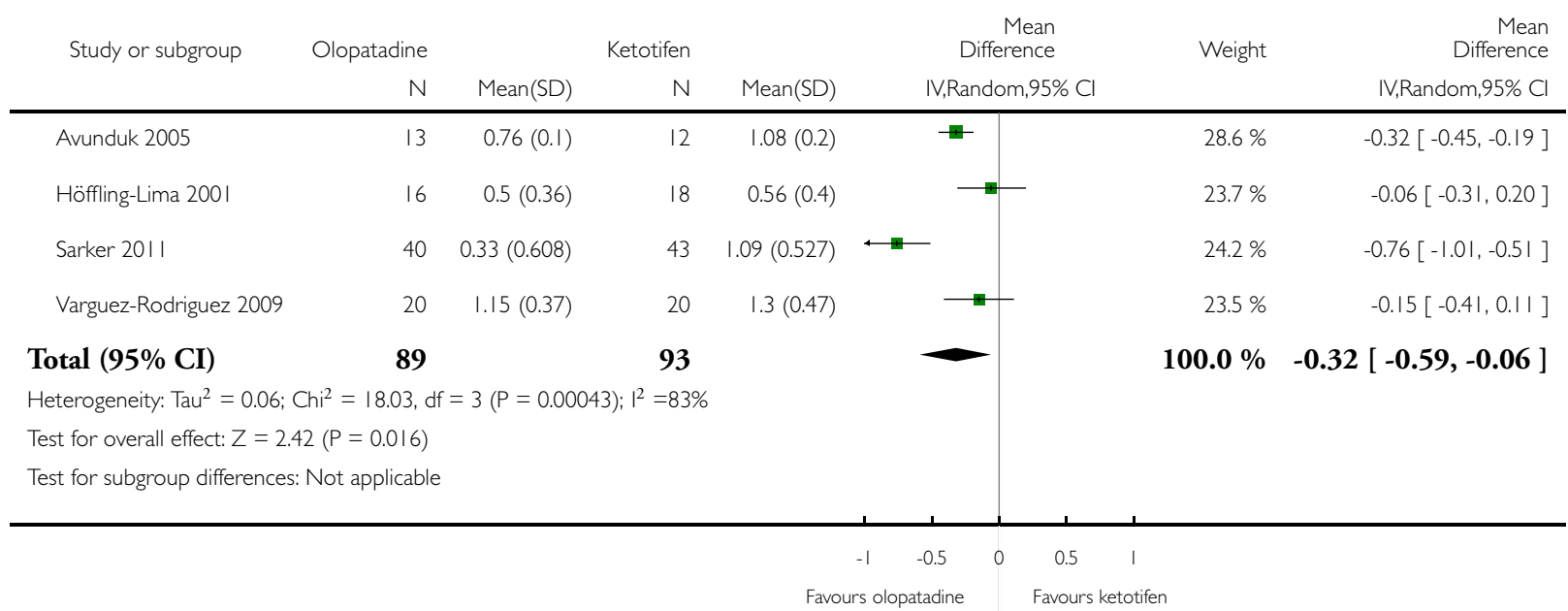




\section{Analysis I.2. Comparison I Olopatadine versus ketotifen, Outcome 2 Tearing at 14 days.}

Review: Topical antihistamines and mast cell stabilisers for treating seasonal and perennial allergic conjunctivitis

Comparison: I Olopatadine versus ketotifen

Outcome: 2 Tearing at 14 days

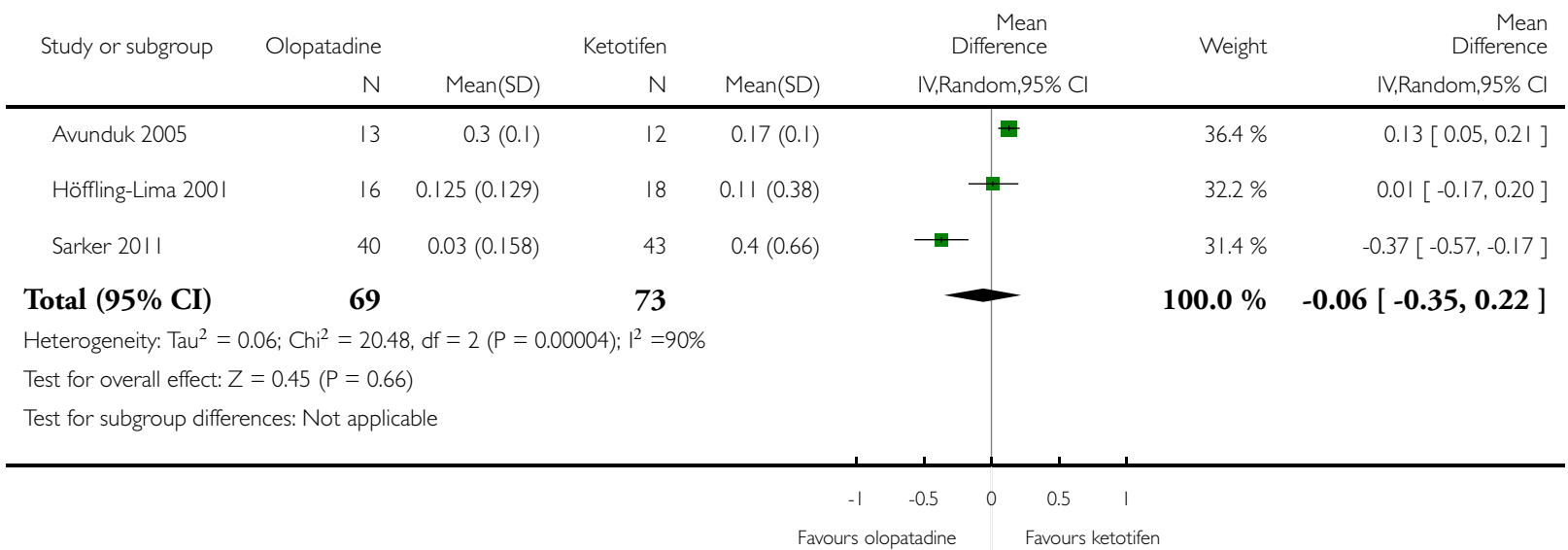

\section{A D D I T IONAL TABLES}

Table 1. Serious adverse events and withdrawals

\begin{tabular}{|c|c|c|c|}
\hline Study & Serious adverse events & $\begin{array}{l}\text { Number withdrawn due to side } \\
\text { effects }\end{array}$ & $\begin{array}{l}\text { Treatment arm from which } \\
\text { withdrawn }\end{array}$ \\
\hline Avunduk 2005 & $\begin{array}{l}\text { No serious adverse events re- } \\
\text { ported }\end{array}$ & $\begin{array}{l}\text { No participants recorded to have } \\
\text { withdrawn }\end{array}$ & N/A \\
\hline Azevedo 1991 & $\begin{array}{l}\text { No serious adverse events re- } \\
\text { ported }\end{array}$ & 1 participant withdrawn & 1 from placebo group \\
\hline Canonica 2003 & $\begin{array}{l}\text { No serious adverse events re- } \\
\text { ported }\end{array}$ & 2 participants withdrawn & $\begin{array}{l}1 \text { from azelastine and } 1 \text { from } \\
\text { placebo }\end{array}$ \\
\hline Carr 2013 & $\begin{array}{l}1 \text { serious adverse event reported } \\
\text { unrelated to the treatment }\end{array}$ & 11 participants withdrawn & $\begin{array}{l}4 \text { from bepotastine besilate and } 7 \\
\text { from placebo }\end{array}$ \\
\hline Davies 1993 & $\begin{array}{l}\text { No serious adverse events re- } \\
\text { ported }\end{array}$ & 5 participants withdrawn & $\begin{array}{l}2 \text { from levocabastine group and } 3 \\
\text { from placebo }\end{array}$ \\
\hline Fujishima 2008 & $\begin{array}{l}\text { No serious adverse events re- } \\
\text { ported }\end{array}$ & $\begin{array}{l}\text { No participants recorded to have } \\
\text { withdrawn }\end{array}$ & N/A \\
\hline
\end{tabular}


Table 1. Serious adverse events and withdrawals (Continued)

\begin{tabular}{|c|c|c|c|}
\hline Giede-Tuch 1998 & $\begin{array}{l}\text { No serious adverse events re- } \\
\text { ported }\end{array}$ & $\begin{array}{l}\text { No participants withdrawn due to } \\
\text { side effects }\end{array}$ & N/A \\
\hline Graue 1994 & $\begin{array}{l}\text { No serious adverse events re- } \\
\text { ported }\end{array}$ & 2 participants withdrawn & $\begin{array}{l}1 \text { from levocabastine and } 1 \text { from } \\
\text { placebo }\end{array}$ \\
\hline Hechanova 1984 & $\begin{array}{l}\text { No serious adverse events re- } \\
\text { ported }\end{array}$ & 12 participants withdrawn & $\begin{array}{l}2 \text { from cromoglycate group and } \\
10 \text { from placebo group }\end{array}$ \\
\hline Höffling-Lima 2001 & $\begin{array}{l}\text { No serious adverse events re- } \\
\text { ported }\end{array}$ & $\begin{array}{l}\text { No participants recorded to have } \\
\text { withdrawn }\end{array}$ & N/A \\
\hline James 2003 & $\begin{array}{l}\text { No serious adverse events re- } \\
\text { ported }\end{array}$ & $\begin{array}{l}4 \text { participants withdrawn due to } \\
\text { side effects }\end{array}$ & $\begin{array}{l}1 \text { from azelastine, } 2 \text { from cromo- } \\
\text { glycate, and } 1 \text { from placebo }\end{array}$ \\
\hline Katelaris 2002 & $\begin{array}{l}\text { No serious adverse events re- } \\
\text { ported }\end{array}$ & 2 participants withdrawn & $\begin{array}{l}1 \text { from olopatadine group, } 1 \text { from } \\
\text { cromolyn group }\end{array}$ \\
\hline Kidd 2003 & $\begin{array}{l}4 \text { serious adverse events: 1) per- } \\
\text { sistent photophobia and 2) con- } \\
\text { junctivitis with corneal ulcer }\end{array}$ & $\begin{array}{l}38 \text { discontinued due to side ef- } \\
\text { fects }\end{array}$ & $\begin{array}{l}8 \text { from ketotifen, } 15 \text { from levo- } \\
\text { cabastine, } 15 \text { from placebo }\end{array}$ \\
\hline Lanier 2001 & $\begin{array}{l}\text { No serious adverse events re- } \\
\text { ported }\end{array}$ & $\begin{array}{l}\text { No participants recorded to have } \\
\text { withdrawn }\end{array}$ & N/A \\
\hline Leino 1992 & $\begin{array}{l}\text { No serious adverse events re- } \\
\text { ported }\end{array}$ & 4 participants withdrawn & $\begin{array}{l}3 \text { from nedocromil and } 1 \text { from } \\
\text { cromoglycate }\end{array}$ \\
\hline Lenhard 1997 & $\begin{array}{l}\text { No serious adverse events re- } \\
\text { ported }\end{array}$ & 4 participants withdrawn & $\begin{array}{l}2 \text { from azelastine group and } 2 \\
\text { from placebo group }\end{array}$ \\
\hline McCabe 2012 & $\begin{array}{l}\text { No serious adverse events re- } \\
\text { ported }\end{array}$ & $\begin{array}{l}\text { No participants recorded to have } \\
\text { withdrawn }\end{array}$ & N/A \\
\hline Melamed 1994 & $\begin{array}{l}\text { No serious adverse events re- } \\
\text { ported }\end{array}$ & $\begin{array}{l}\text { No participants recorded to have } \\
\text { withdrawn due to side effects }\end{array}$ & N/A \\
\hline Melamed 2000 & $\begin{array}{l}\text { No serious adverse events re- } \\
\text { ported }\end{array}$ & $\begin{array}{l}\text { No participants recorded to have } \\
\text { withdrawn due to side effects }\end{array}$ & N/A \\
\hline Moller 1994 & $\begin{array}{l}\text { No serious adverse events re- } \\
\text { ported }\end{array}$ & $\begin{array}{l}\text { No participants recorded to have } \\
\text { withdrawn due to side effects }\end{array}$ & N/A \\
\hline Nazarov 2003 & $\begin{array}{l}\text { No serious adverse events re- } \\
\text { ported }\end{array}$ & $\begin{array}{l}1 \text { participant withdrawn due to } \\
\text { side effects }\end{array}$ & $\begin{array}{l}1 \text { participant from azelastine } \\
\text { group }\end{array}$ \\
\hline
\end{tabular}

Topical antihistamines and mast cell stabilisers for treating seasonal and perennial allergic conjunctivitis (Review) 
Table 1. Serious adverse events and withdrawals (Continued)

\begin{tabular}{|c|c|c|c|}
\hline Petzold a,b,c 2002 & $\begin{array}{l}\text { No serious adverse events re- } \\
\text { ported }\end{array}$ & $\begin{array}{l}\text { No participants recorded to have } \\
\text { withdrawn due to side effects }\end{array}$ & N/A \\
\hline Sabbah 1998 & $\begin{array}{l}\text { No serious adverse events re- } \\
\text { ported }\end{array}$ & 1 participant withdrawn & $\begin{array}{l}1 \text { participant from azelastine } \\
\text { group }\end{array}$ \\
\hline Sarker 2011 & $\begin{array}{l}\text { No serious adverse events re- } \\
\text { ported }\end{array}$ & $\begin{array}{l}\text { No mention of participants with- } \\
\text { drawing due to side effects }\end{array}$ & N/A \\
\hline Secchi a 2000 & $\begin{array}{l}\text { No serious adverse events re- } \\
\text { ported }\end{array}$ & $\begin{array}{l}1 \text { participant discontinued due to } \\
\text { side effects }\end{array}$ & 1 participant from emedastine \\
\hline Secchi b 2000 & $\begin{array}{l}\text { No serious adverse events re- } \\
\text { ported }\end{array}$ & $\begin{array}{l}\text { No mention of participants with- } \\
\text { drawing due to side effects }\end{array}$ & N/A \\
\hline Trinquand 1999 & $\begin{array}{l}\text { No serious adverse events re- } \\
\text { ported }\end{array}$ & $\begin{array}{l}\text { No mention of participants with- } \\
\text { drawing due to side effects }\end{array}$ & N/A \\
\hline Varguez-Rodriguez 2009 & $\begin{array}{l}\text { No serious adverse events re- } \\
\text { ported }\end{array}$ & $\begin{array}{l}\text { No participants recorded to have } \\
\text { withdrawn }\end{array}$ & N/A \\
\hline Verin 2001 & $\begin{array}{l}\text { No serious adverse events re- } \\
\text { ported }\end{array}$ & 3 participants withdrawn & $\begin{array}{l}3 \text { participants from emedastine } \\
\text { group }\end{array}$ \\
\hline Wertheimer 1997 & $\begin{array}{l}\text { No serious adverse events re- } \\
\text { ported }\end{array}$ & $\begin{array}{l}\text { No participants recorded to have } \\
\text { withdrawn due to side effects }\end{array}$ & N/A \\
\hline
\end{tabular}

N/A: not applicable

\section{A P PENDICES}

\section{Appendix I. Listed medications and mechanism of action}

Antazoline - pure antihistamine

Azelastine - antihistamine + mast cell stabiliser

Bepotastine besilate - antihistamine + mast cell stabiliser

Carebastine - developing drug - pure antihistamine

Levocabastine - pure antihistamine

Emedastine - pure antihistamine

Ketotifen - antihistamine + mast cell stabiliser

Olopatadine - antihistamine + mast cell stabiliser

Epinastine - antihistamine + mast cell stabiliser

Sodium chromoglycate - antihistamine + mast cell stabiliser

Nedocromil sodium - antihistamine + mast cell stabiliser

Topical antihistamines and mast cell stabilisers for treating seasonal and perennial allergic conjunctivitis (Review)

Copyright @ 2015 The Cochrane Collaboration. Published by John Wiley \& Sons, Ltd. 
Lodoxamide - antihistamine + mast cell stabiliser

Tranilast - developing drug - antihistamine + mast cell stabiliser

\section{Appendix 2. CENTRAL search strategy}

\#1 MeSH descriptor Conjunctivitis, Allergic

\#2 conjunctivitis

\#3 pollen near/3 allerg*

\#4 hayfever

\#5 hay near $/ 2$ fever

\#6 (\#1 OR \#2 OR \#3 OR \#4 OR \#5)

\#7 MeSH descriptor Histamine H1 Antagonists

\#8 antihistamin* or anti-histamin*

\#9 antazolin*

$\# 10$ azelastin*

\#11 bepotastin* or Senju

\#12 carebastin*

\#13 levocabastin*

\#14 emedastin*

\#15 MeSH descriptor Ketotifen

\#16 ketotifen*

\#17 olopatadin*

\#18 epinastin*

\#19 MeSH descriptor Cromolyn Sodium

\#20 sodium chromoglycate

\#21 MeSH descriptor Nedocromil

\#22 nedocromil sodium

\#23 lodoxamid*

\#24 tranilast*

\#25 (\# OR \#8 OR \#9 OR\#10 OR \#11 OR \#12 OR \#13 OR \#14 OR \#15 OR \#16 OR \#17 OR \#18 OR \#19 OR \#20 OR \#21 OR

\#22 OR \#23 OR \#24)

\#26 (\#6 AND \#25)

\section{Appendix 3. MEDLINE (OvidSP) search strategy}

1. randomized controlled trial.pt.

2. (randomized or randomised).ab,ti.

3. placebo.ab,ti.

4. dt.fs.

5. randomly.ab,ti.

6. trial.ab,ti.

7. groups.ab,ti.

8. or/1-7

9. exp animals/

10. exp humans/

11. 9 not (9 and 10)

12. 8 not 11

13. conjunctivitis, allergic/

14. conjunctivitis.tw.

15. (pollen adj3 allerg\$).tw.

16. hayfever.tw.

17. (hay adj2 fever).tw.

Topical antihistamines and mast cell stabilisers for treating seasonal and perennial allergic conjunctivitis (Review)

Copyright $\odot 2015$ The Cochrane Collaboration. Published by John Wiley \& Sons, Ltd. 
18. or/13-17

19. exp histamine h1 antagonists/

20. (antihistamin\$ or anti-histamin\$).tw.

21. antazolin\$.tw.

22. azelastin $\$$.tw.

23. (bepotastin\$ or Senju).tw.

24. carebastin\$.tw.

25. levocabastin $\$$. tw.

26. emedastin\$.tw.

27. Ketotifen/

28. ketotifen\$.tw.

29. olopatadin\$.tw.

30. epinastin $\$$.tw.

31. Cromolyn Sodium/

32. sodium chromoglycate.tw.

33. Nedocromil/

34. nedocromil sodium.tw.

35. lodoxamid \$.tw.

36. tranilast\$.tw.

37. or/19-36

38.18 and 37

39. 12 and 38

The search filter for trials at the beginning of the MEDLINE strategy is from the published paper by Glanville (Glanville 2006).

\section{Appendix 4. EMBASE (OvidSP) search strategy}

1. exp randomized controlled trial/

2. exp randomization/

3. exp double blind procedure/

4. exp single blind procedure/

5. random $\$$.tw.

6. or/1-5

7. (animal or animal experiment).sh.

8. human.sh.

9.7 and 8

10.7 not 9

11. 6 not 10

12. exp clinical trial/

13. (clin $\$$ adj3 trial $\$)$.tw.

14. ((singl\$ or doubl\$ or trebl\$ or tripl\$) adj3 (blind\$ or mask\$)).tw.

15. exp placebo/

16. placebo $\$ . t w$.

17. random $\$$.tw.

18. exp experimental design/

19. exp crossover procedure/

20. exp control group/

21. exp latin square design/

22. or/12-21

23. 22 not 10

24. 23 not 11

25. exp comparative study/

26. exp evaluation/

Topical antihistamines and mast cell stabilisers for treating seasonal and perennial allergic conjunctivitis (Review)

Copyright $\odot 2015$ The Cochrane Collaboration. Published by John Wiley \& Sons, Ltd. 
27. exp prospective study/

28. (control\$ or prospectiv\$ or volunteer\$).tw.

29. or $/ 25-28$

30. 29 not 10

31.30 not (11 or 23$)$

32. 11 or 24 or 31

33. exp allergic conjunctivitis/

34. conjunctivitis.tw.

35. (pollen adj3 allerg\$).tw.

36. hayfever.tw.

37. (hay adj2 fever).tw.

38. or/33-37

39. histamine $\mathrm{H} 1$ receptor antagonist/

40. (antihistamin\$ or anti-histamin\$).tw.

41. antazoline/

42. antazolin\$.tw.

43. azelastine/

44. azelastin\$.tw.

45. carebastine/

46. (bepotastin\$ or Senju).tw.

47. carebastin\$.tw.

48. levocabastine/

49. levocabastin\$.tw.

50. emedastine/

51. emedastin\$.tw.

52. ketotifen/

53. ketotifen $\$$.tw.

54. olopatadine/

55. olopatadin\$.tw.

56. epinastine/

57. epinastin \$.tw.

58. cromoglycate disodium/

59. sodium chromoglycate.tw.

60 . nedocromil sodium/

61. nedocromil sodium.tw.

62. lodoxamide/

63. lodoxamid\$.tw.

64. tranilast/

65. tranilast $\$$.tw.

66. or/39-65

67.38 and 66

68.32 and 67 


\section{Appendix 5. metaRegister of Controlled Trials search strategy}

allergic conjunctivitis and antihistamine

\section{Appendix 6. ClinicalTrials.gov search strategy}

allergic conjunctivitis AND antihistamine

\section{Appendix 7. ICTRP search strategy}

allergic conjunctivitis AND antihistamine

\section{CONTRIBUTIONSOFAUTHORS}

Conceiving the review: MSM, MC, AAB

Designing the review: MSM, MC, AAB, NS

Drafting the protocol: MSM, MC, AAB

Coordinating the review: $\mathrm{MC}$

Undertaking electronic and manual searches: MSM, MC, MZM

Screening search results: MSM, MC, MZM

Organising retrieved papers against inclusion criteria: MC

Appraising methodological quality of papers: MC, AAB, NS

Abstracting data from papers: MC, MSM, MZM, NS, AAB

Data management of the review: MC

Entering data into RevMan: MC, NS

Analysing and presenting results: MC, NS, AAB

Interpreting results: $\mathrm{MC}, \mathrm{NS}, \mathrm{AAB}$

Writing the review: MC, NS, AAB

\section{DECLARATIONSOF INTEREST}

We declare that there is no conflict of interest or financial interest in this systematic review by any of the review authors. 


\section{SOURCES OF SUPPORT}

\section{Internal sources}

- No sources of support supplied

\section{External sources}

- National Institutute of Health Research (NIHR), UK.

Richard Wormald, Co-ordinating Editor for Cochrane Eyes and Vision (CEV) acknowledges financial support for his CEV research sessions from the Department of Health through the award made by the National Institute for Health Research to Moorfields Eye Hospital NHS Foundation Trust and UCL Institute of Ophthalmology for a Specialist Biomedical Research Centre for Ophthalmology.

- The NIHR also funds the CEV Editorial Base in London.

The views expressed in this publication are those of the authors and not necessarily those of the NIHR, NHS, or the Department of Health.

\section{DIFFERENCES BETWEEN PROTOCOLANDREVIEW}

None.

\section{NOTES}

The current protocol is an update of a published protocol by Purohit G, Jayabalan P, Ratnasingham K, Patter A. Topical antihistamines for seasonal and perennial allergic conjunctivitis. Cochrane Database of Systematic Reviews 2006, Issue 1. The 'Background' and 'Objectives' sections of the protocol have been updated, and the 'Methods' section has been rewritten in line with updated Cochrane methodology. The protocol has also been peer reviewed again. 